\title{
REAL-TIME FAULT DETECTION AND RECONFIGURATION OF A THREE-PHASE ELECTRIC MOTOR DRIVE
}

\author{
by \\ Danyal Mohammadi
}

\author{
A dissertation \\ submitted in partial fulfillment \\ of the requirements for the degree of
}

Doctor of Philosophy in Electrical and Computer Engineering

Boise State University

May 2019 
(C) 2019

Danyal Mohammadi

ALL RIGHTS RESERVED 


\section{BOISE STATE UNIVERISTY GRADUATE COLLEGE DEFENSE COMMITTEE AND FINAL READING APPROVALS}

of the dissertation submitted by

Danyal Mohammadi

Dissertation Title: Real-Time Fault Detection and Reconfiguration of a Three-Phase Electric Motor Drive

Date of Oral Examination: 12th April 2019

The following individuals read and discussed the dissertation submitted by student Danyal Mohammadi, and they evaluated his presentation and response to questions during the final oral examination. They found that the student passed the final oral examination.

Said Ahmed-Zaid, Ph.D. Chair, Supervisory Committee

Nader Rafla, Ph.D. Member, Supervisory Committee

Thad Welch, Ph.D. Member, Supervisory Committee

The final reading approval of the dissertation was granted by Said Ahmed-Zaid, Ph.D., Chair of the Supervisory Committee. The dissertation was approved by the Graduate College. 


\begin{abstract}
Variable-frequency drives (VFDs) are widely used for control of electrical machines such as induction motors (IMs) or permanent-magnet synchronous motors (PMSMs). Similar to other electrical devices, these drives are subject to failure. Several types of faults are associated with VFDs. For instance, faults such as open-switch faults, and short-circuit switch faults are the two common faults in VFDs. These faults can yield catastrophic consequences if proper remedial action is not taken.

A unique remedial topology for the post-fault period and a new pulse width modulation (PWM) strategy are proposed so that not only the motor drive can continue the operation, but also this new method reduces the common-mode voltage of the three-phase inverter.

In this work, switch failures are investigated, and a new solution to detect openswitch faults are proposed using an algorithm based on current analyses. Extensive simulations are done to simulate a real system response during the post-fault phase on different operating points and at various torque loads. These simulation results were used to develop a detection algorithm. These results were also verified using an experimental setup. The proposed method offers an efficient solution for fault detection in VFDs. First, a fast response detection of a failure in an inverter is possible. Second, it provides an alternative back-up method for fault detection in electric drive systems.
\end{abstract}




\section{TABLE OF CONTENTS}

ABSTRACT $\ldots \ldots \ldots \ldots \ldots \ldots \ldots \ldots \ldots \ldots \ldots \ldots \ldots \ldots \ldots \ldots \ldots$

LIST OF TABLES $\ldots \ldots \ldots \ldots \ldots \ldots \ldots \ldots \ldots \ldots \ldots \ldots \ldots \ldots \ldots \ldots \ldots$

LIST OF FIGURES $\ldots \ldots \ldots \ldots \ldots \ldots \ldots \ldots \ldots \ldots \ldots \ldots \ldots \ldots \ldots \ldots$

LIST OF ABBREVIATIONS $\ldots \ldots \ldots \ldots \ldots \ldots \ldots \ldots \ldots \ldots$ xv

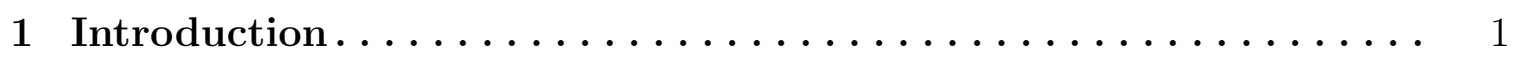

2 Basics of Induction Motor Control $\ldots \ldots \ldots \ldots \ldots \ldots \ldots$

2.1 Induction Motor Model $\ldots \ldots \ldots \ldots \ldots \ldots \ldots \ldots \ldots \ldots$

2.1.1 Induction motor model in the the $a b c$-reference frame $\ldots \ldots .6$

2.1.2 Induction motor model in an arbitrary $q d 0$ reference frame . . . 11

2.2 Induction Motor Torque Equation . . . . . . . . . . . . . 14

2.3 Field-Oriented Control Method ........................ 16

2.4 SVPWM Background $\ldots \ldots \ldots \ldots \ldots \ldots \ldots \ldots \ldots \ldots \ldots \ldots \ldots \ldots \ldots \ldots \ldots \ldots$

2.4.1 Angle and Voltage Reference Vector ................ 19

2.4 .2 Modulation Index . . . . . . . . . . . . . . . . . . . 22

2.4 .3 Time Calculations ......................... 22

2.5 Basic Operation of a Three-Phase Inverter . . . . . . . . . . . 24

2.5.1 Common-Mode Voltage in Two-Level Inverter . . . . . . . . . 25

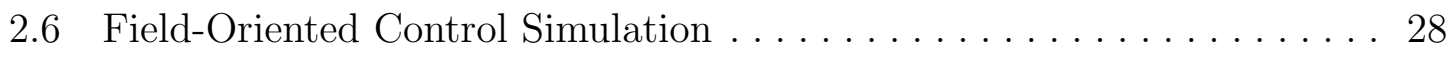




\section{Active Common-Mode Voltage Reduction in a Fault-Tolerant Three-}

Phase Inverter . . . . . . . . . . . . . . . . . 30

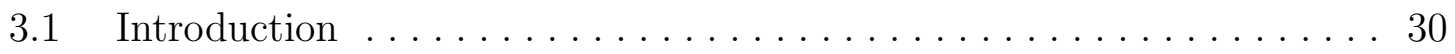

3.2 Literature Review on Common-Mode Voltage $\ldots \ldots \ldots \ldots \ldots \ldots$

3.3 Literature Review of the Fault-Tolerant Systems . . . . . . . . . . 35

3.3.1 Two-Phase Operation as a Remedial Strategy . . . . . . . . . . 36

3.3.2 Utilizing Redundant Leg Remedial Strategies ... . . . . . . . . . 39

3.4 A Proposed New Remedial Strategy . . . . . . . . . . . . . . . . . . . 40

3.4.1 Pre-Fault Operation Mode . . . . . . . . . . . . . . . . . 41

3.4 .2 Reconfiguration Mode ...................... 41

3.4.3 Post-Fault Operation Mode....................... 42

3.4.4 Filter Design .............................. 43

3.5 Simulation Results $\ldots \ldots \ldots \ldots \ldots \ldots \ldots \ldots \ldots \ldots \ldots \ldots$

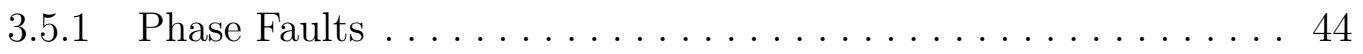

$3.5 .2 \quad$ Switch Faults $\ldots \ldots \ldots \ldots \ldots \ldots \ldots \ldots \ldots \ldots \ldots$

3.6 Conclusion ............................. 48

4 New Open-Switch Fault Detection Method ................ 49

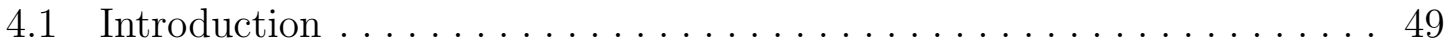

4.2 Literature Review of the Open-Switch Fault . . . . . . . . . . . . 52

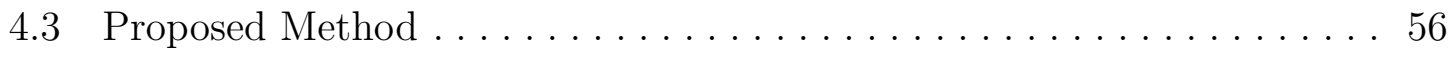

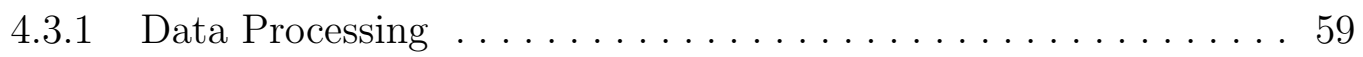

4.3.2 Covariance Computation ...................... 60

4.3 .3 Fault Signatures . . . . . . . . . . . . . . . . . . . . 62

4.4 Results .............................. 63 
4.4.1 Simulation Results . . . . . . . . . . . . . . . . 63

4.4.2 Experimental Results . . . . . . . . . . . . . . . . . 65

4.5 Extension to a Five-Phase Drive System $\ldots \ldots \ldots \ldots \ldots \ldots \ldots$

4.5.1 A-COV Methodology for a Five-Phase Motor . . . . . . . . . 69

4.5.2 Simulation Results .......................... 74

4.6 Conclusion $\ldots \ldots \ldots \ldots \ldots \ldots \ldots \ldots \ldots \ldots \ldots \ldots \ldots \ldots \ldots$

5 Design Implementation on $\mathrm{SoC} \ldots \ldots \ldots \ldots \ldots \ldots \ldots \ldots \ldots$

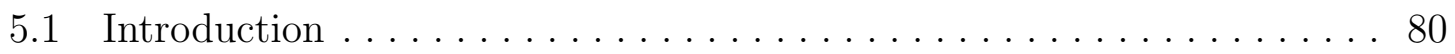

5.2 Literature Review of Hardware/Software codesign . . . . . . . . . . 81

5.3 The Zynq All Programmable $\mathrm{SoC} \ldots \ldots \ldots \ldots \ldots \ldots$

5.4 Algorithm Codesign Development . . . . . . . . . . . . . . . 84

$5.4 .1 \quad$ Hardware. . . . . . . . . . . . . . . . . . . . . . . 85

$5.4 .2 \quad$ Software $\ldots \ldots \ldots \ldots \ldots \ldots \ldots \ldots \ldots \ldots \ldots \ldots$

5.5 Design Evaluation.......................... 87

5.5 .1 Experimental Setup . . . . . . . . . . . . . . . 88

$5.5 .2 \quad$ Experimental Results . . . . . . . . . . . . . . . . 88

6 Experimental Setup $\ldots \ldots \ldots \ldots \ldots \ldots \ldots \ldots \ldots \ldots \ldots$

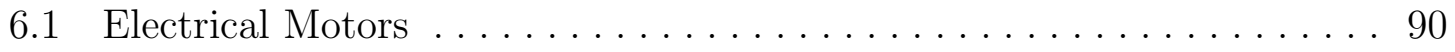

6.2 Three-Phase Inverter $\ldots \ldots \ldots \ldots \ldots \ldots \ldots \ldots \ldots \ldots \ldots \ldots \ldots$

6.2.1 Board Design Specifications $\ldots \ldots \ldots \ldots \ldots \ldots \ldots \ldots \ldots$

6.3 PWM Signals . ............................ 98

6.4 Computational Unit . . . . . . . . . . . . . . . . . . . . 98

6.5 Logging Data . . . . . . . . . . . . . . . . . . . . . 99

6.6 Conclusion ................................ 99 
7 Conclusion and Future Work $\ldots \ldots \ldots \ldots \ldots \ldots \ldots \ldots \ldots \ldots \ldots \ldots$

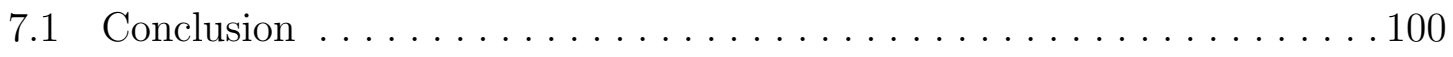

$7.2 \quad$ Future Work . . . . . . . . . . . . . . . . . . . . 101

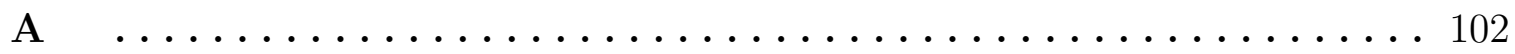

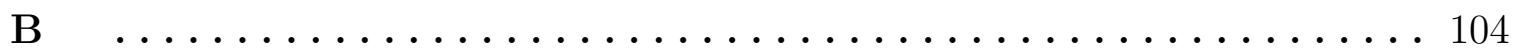

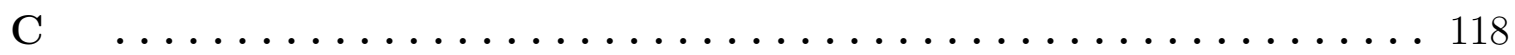

REFERENCES.......................... 123 


\section{LIST OF TABLES}

2.1 Common-mode voltage for all vectors in the SVPWM . . . . . . . . . 28

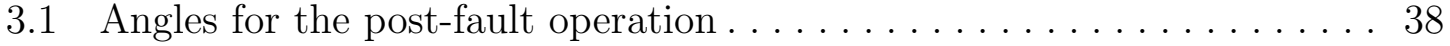

3.2 Parameters and values used in the simulation $\ldots \ldots \ldots \ldots \ldots \ldots \ldots 4$

4.1 Fault detection timing for different methods in literature . . . . . . . 56

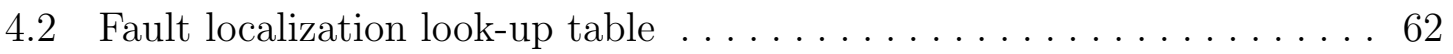

4.3 Fault localization look-up table for a five-phase inverter . . . . . . . . . 73

4.4 Test and evaluation parameter definition $\ldots \ldots \ldots \ldots \ldots \ldots \ldots$

5.1 Hardware platform setup parameter values $\ldots \ldots \ldots \ldots \ldots \ldots$

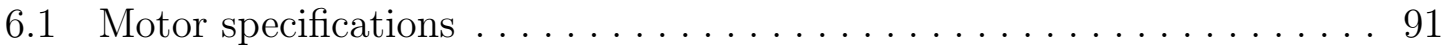

A.1 Simulation parameter values . . . . . . . . . . . . . . . . . . . 103 


\section{LIST OF FIGURES}

1.1 Circuit of the VFD and a motor with load attached and a few possible fault locations .......................... 2

2.1 Squirrel-cage induction motor with three-phase $a b c$, arbitrary $(q d 0)$,

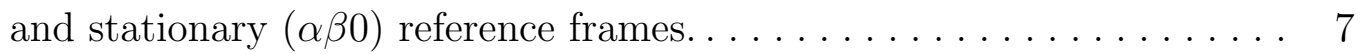

2.2 Schematic of the field-oriented control with Space-Vector PWM . . . . . 18

2.3 Space-vector diagram for a two-level voltage inverter . . . . . . . . . . . . . 19

2.4 Illustration of three-phase current in $a b c$ and $\alpha \beta 0$-coordinates . . . . . 21

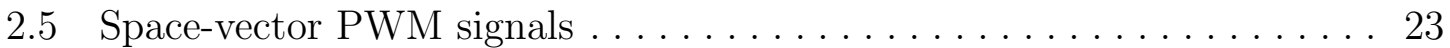

2.6 Space-vector PWM schematic for calculation of $T_{0}, T_{1}$, and $T_{2} \ldots \ldots 24$

2.7 SVPWM signals for three-phase voltage source inverter . . . . . . . . . . 24

2.8 The schematic of one leg of the three-phase inverter. . . . . . . . . 25

2.9 Operation of dead-time in one leg of the inverter for both positive and

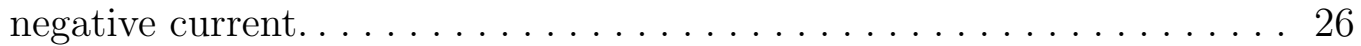

2.10 Common-mode voltage signals in a three-phase inverter . . . . . . . . 27

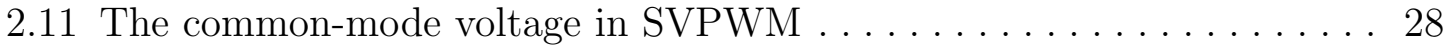

2.12 Simulation results with the FOC; speed, electromagnetic torque, currents, and DC-link voltage. . . . . . . . . . . . . . . . . . . . 29

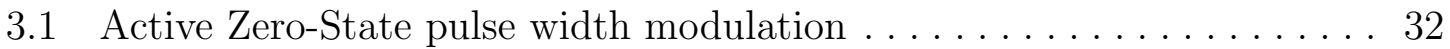


3.2 Common-mode voltage and switches status, AZSPWM modulation in

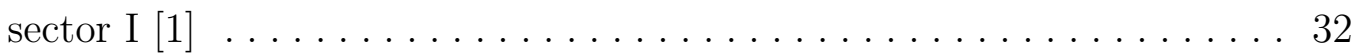

3.3 The Circuitry of the four-leg inverter showing the proposed method for CMV reduction.

3.4 Voltage of the line to midpoint DC-link; common-mode voltage reduc-

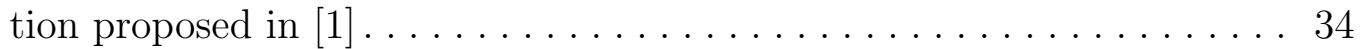

3.5 Common-mode voltage using AZSPWM in different topologies . . . . . . 35

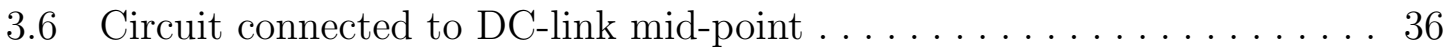

3.7 The neutral $(\mathrm{N})$ of the circuit connected to the DC-link mid-point. . . . 37

3.8 Inverter circuit with a redundant leg $[2] \ldots \ldots \ldots \ldots$. . . . . . . . 39

3.9 The circuitry of the proposed topology $\ldots \ldots \ldots \ldots \ldots \ldots$

3.10 Post-Fault circuit for short-circuited or phase-open (two-phase operation) 42

3.11 Post-Fault circuit for switch-short or open-switch . . . . . . . . . . 42

3.12 Open-circuit phase $a$ failure $\ldots \ldots \ldots \ldots \ldots \ldots \ldots \ldots$

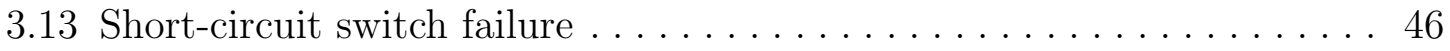

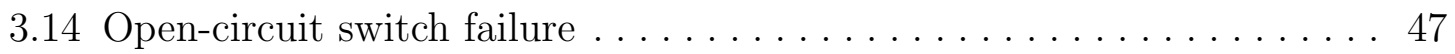

3.15 Comparison of CMV and CMC ................... 48

3.16 Four-leg inverter currents and THD $\ldots \ldots \ldots \ldots \ldots \ldots \ldots$

4.1 Simplified three-phase two-level inverter with fault at switch $S_{a} \ldots \ldots 5$

4.2 Simulation of three-phase current in pre- and post-fault period . . . . . . 51

4.3 Healthy and faulty current in $\alpha \beta$-plane for each fault; $\omega_{r}=900 \mathrm{rpm}$

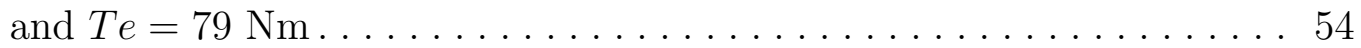

4.4 Fault angle illustration with respect to the phase $a \ldots \ldots \ldots \ldots 7$ 
4.5 Experimental: the normalized three-phase current during pre- and post-fault at switch $S_{1} \ldots \ldots \ldots \ldots \ldots \ldots \ldots$

4.6 Experimental: co-variance value and its mean during pre- and post-fault 61

4.7 Experimental results: (a) three-phase currents and their mean; (b) absolute value of the co-variance; (c) fault flag for park vector and the proposed vector method $\ldots \ldots \ldots \ldots \ldots \ldots \ldots$

4.8 Simulation results of the detection time for different fault-angle at different operating frequencies at switch $S 1 \ldots \ldots \ldots \ldots 6$

4.9 Experimental results of the detection time for different firing angle at different operating frequencies at switch $S 1 \ldots \ldots \ldots \ldots 6$

4.10 Detection time in percentage of a cycle for different firing angle at different operating frequencies at switch $S 1 \ldots \ldots \ldots \ldots$. . . . 67

4.11 Simulation of the $C_{X Y}$ and five-phase motor currents during pre- and

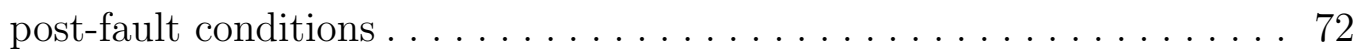

4.12 Detection logic gates for an open-switch fault at $S_{3} \ldots \ldots \ldots 73$

4.13 Simulation results of detection time for different frequency for a five-

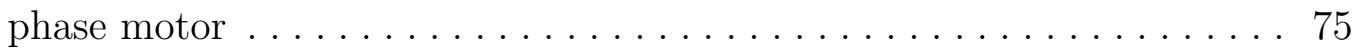

4.14 Simulation of A-COV and fault signal with random torque command

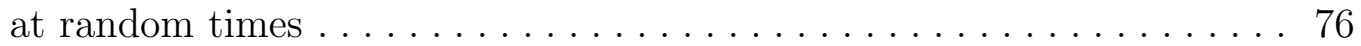

5.1 Power electronic components and control architecture of the design . . . 85

5.2 Experimental results from top to bottom: operating frequency command, current phase $a$ and $b$ of the motor, voltage of the DC-Link $\ldots 89$

6.1 AC Motor and DC Motor coupled to each other . . . . . . . . . . 91

6.2 Printed circuit board of the three-phase inverter . . . . . . . . . . . 92 
6.3 Layout of printed circuit board of the three-phase inverter . . . . . . . 93

6.4 The circuitry of the sensor of the phase $b$ current $\ldots \ldots \ldots \ldots \ldots \ldots 4$

6.5 Measured voltage vs. applied current in the current sensor . . . . . . . . 95

6.6 The circuitry of the voltage sensing of the DC-Link . . . . . . . . . . 95

6.7 The 5 DIN connections of the two power supplies $\ldots \ldots \ldots \ldots$

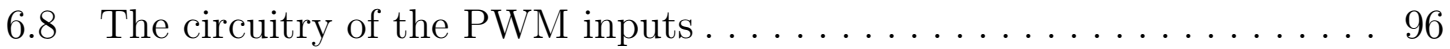

6.9 Three-phase gate driver and switches for one leg . . . . . . . . . . 97

6.10 Three-Phase gate driver and switches for one leg . . . . . . . . . 97

6.11 SVPWM signals for the three-phase inverter during pre- and post-fault 98

6.12 dSPACE ControlDesk diagram of user interface for real-time data logging 99

B.1 The screenshot of the simulation of the module $\ldots \ldots \ldots \ldots \ldots \ldots$

C.1 Reliability no. 2: simulations of random torque commands at random times... . . . . . . . . . . . . . . . . . . . . . . . . . 119

C.2 Reliability no. 3: simulations of random torque commands at random times. . . . . . . . . . . . . . . . . . . . . . . . 120

C.3 Reliability no. 4: simulations of random torque commands at random times. . . . . . . . . . . . . . . . . . . . . . . . . . . . . . . . . 121

C.4 Reliability no. 5: simulations of random torque commands at random times. . . . . . . . . . . . . . . . . . . . . . . . . . 122 


\title{
LIST OF ABBREVIATIONS
}

\author{
SVPWM - Space Vector Pulse Width Modulation \\ PWM - Pulse Width Modulation \\ IM - Induction Motor \\ PMSM - Permanent Magnet Synchronous Machine \\ SVM - Support Vector Machine \\ VFD - Variable Frequency Drive \\ FOC - Field-Oriented Control \\ DC - Direct Current
}




\section{CHAPTER 1}

\section{INTRODUCTION}

Variable-frequency drives (VFDs) are essential components in many industries, where they control the speed and electromagnetic torque of an electrical motor. The electric motor can be found in many places, such as, electric vehicles, industrial production lines, and automation industries. Their reliability plays an important role in keeping the system operating and safe. A fault in a VFD can cause an undesired and unexpected interruption. Any interruption of a VFD could lead to an expensive halt of a production line. Fault-tolerant systems provide temporary solutions for the industry to avoid an unwanted stop of the production line. Much research on the topic of fault-tolerant systems is available in which state-of-the-art of fault diagnostic methods are utilized to detect the faults. Fault-tolerant VFDs are designed to minimize such incidents and to power the consumer loads in a more reliable manner. Throughout this work, variable frequency drive and electric motor drive will be used interchangeably.

Any fault-tolerant VFD system requires a fault detection algorithm. Researchers have developed many methods for detecting VFD faults. Faults could happen on the load side and also on the drive side. Faults in a VFD (drive side) could occur in various possible components such as switches, anti-parallel diodes, DC-link capacitors, and LCL filters. Figure 1.1 shows a simplified voltage source inverter VFD system with a DC-link capacitor, a motor with a squirrel cage, and a load attached to the motor 


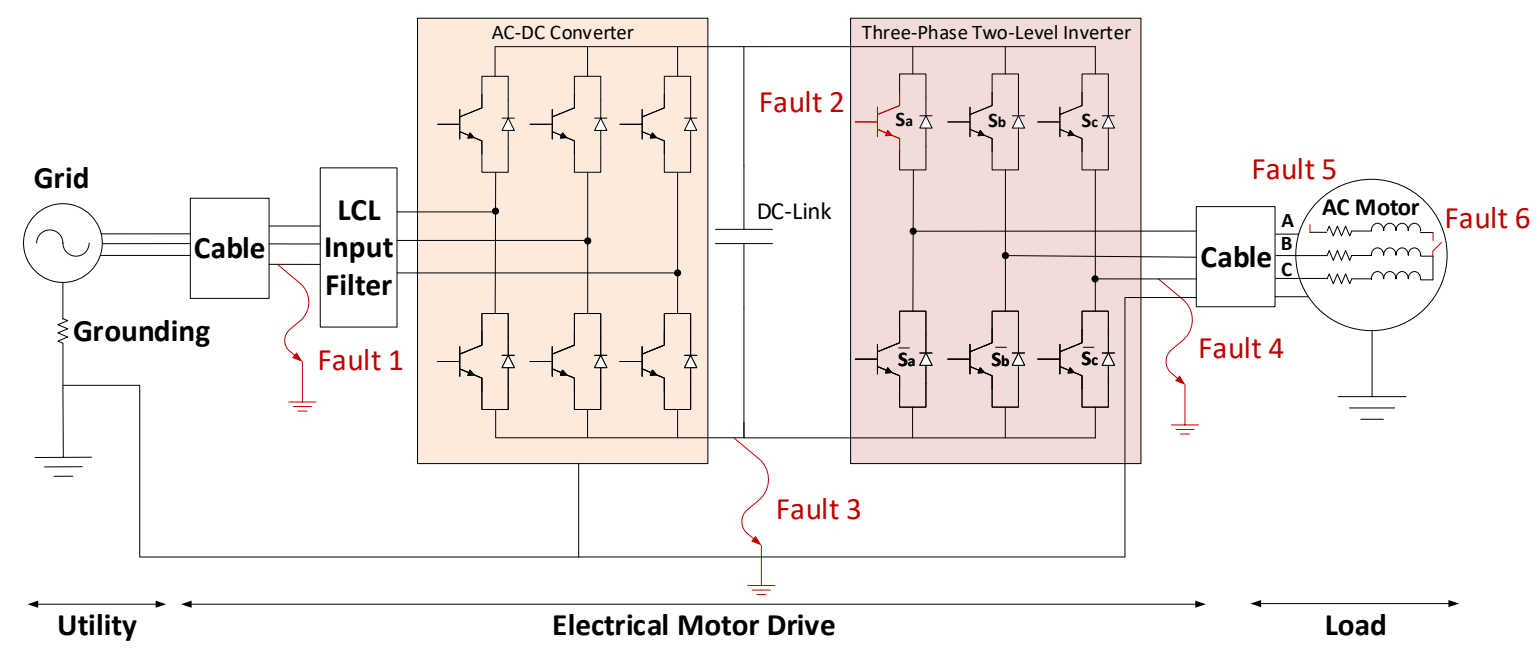

Figure 1.1: Circuit of the VFD and a motor with load attached and a few possible fault locations

and also a few possible fault locations. Some of these faults include the following:

- Fault 1: Input phase short-circuit fault.

- Fault 2: Short-circuited and open-switch fault.

- Fault 3: DC-Link short circuit fault.

- Fault 4: Phase short-circuit fault.

- Fault 5: Induction machine open-phase fault at the motor terminals or anywhere on the motor winding.

- Fault 6: Induction machine open-phase fault.

In this work, the two topics of fault detection algorithm and fault-tolerant circuitry are thoroughly investigated. The first research topic is to find a faster solution for fault detection and fault localization. The fault localization locates which of the six 
switches are faulty. The second research topic is to have a switch redundancy in the VFD. This redundancy allows the VFD to be able to be a fault-tolerant system.

In order to make an electric motor drive fault-tolerant, the circuitry of the electric motor has to be configurable after detecting the fault. The remedial technique in the VFD controller will isolate the faulty switch and will use another switch instead of the faulty switch. For instance, in case of a fault at the top switch of the phase $a\left(S_{a}\right)$, the circuitry of the VFD can be configured so that the faulty $S_{a}$ can be replaced with a back up switch. In a three-phase inverter, if the back up switch is not available, then the whole leg (leg of phase $a$ ) must be isolated and a two or one phase operation of the motor must be deployed to the controller. More details of this type of operation will be explained in the later chapters.

The author in [3] reports a survey where $38 \%$ of the faults in a VFD are caused by power switch failures. Faults on an insulated-gate bipolar transistor (IGBT) or a metal-oxide semiconductor field-effect transistor (MOSFET) switch or the DCLink capacitor in electrical drives could cause explosions inside the drives and their surroundings if they are not detected fast enough. Therefore, detection of such a malfunctioning state of the drive requires diagnostic techniques. These techniques fall into two categories, hardware and software. For instance, fast acting fuses belong to the hardware category and they are used for overcurrent detection and circuit breaking. Software solutions, such as monitoring the output current at the load along with the DC-Link voltage, can be used in order to detect an overcurrent fault. In commercial inverters or drive systems, sensors are usually made available for monitoring the motor currents and the DC-Link voltage. Any additional sensor implemented, such as a voltage sensor for each switch, is considered invasive. Having more sensors would increase the reliability and detection speed of the diagnostic 
algorithms, assuming all of the sensors remain healthy. On the other hand, these extra sensors increase the weight and cost of the system. Therefore, in this work, only the available sensors in the drive are used to develop a diagnostic algorithm.

Fault-tolerant systems are required to have fault detection methods in order to be able to detect a faulty component in the system. Researchers have proposed several fault-tolerant systems $[2,4-7]$ where a post-fault circuit configuration is used to continue the operation of the VFD.

This work presents two improvements in both fault-tolerant systems and also fault detection systems. Each of these topics are presented in separate chapters. Therefore, the literature review of each topic is in their corresponding chapter. As mentioned earlier, the load of the inverter is an induction motor. Therefore, a brief explanation of field-oriented control (FOC) of an induction motor and the basic operation of a three-phase inverter and space-vector pulse width modulation (SVPWM) are given in Chapter 2.

This dissertation is organized as follows. In Chapter 2, a basic operation of an inverter and control of an induction machine are reviewed and simulated. The new fault tolerant circuitry of a three-phase inverter is presented in Chapter 3. In Chapter 4, a new open-switch fault detection algorithm is presented for three-phase and fivephase motor. In chapter 5, the procedure of implementing this algorithm in an FPGA is discussed. The experimental setup developed in this work are explained in detail in Chapter 6. In Chapter 7, the conclusion and future work is presented. 


\section{CHAPTER 2}

\section{BASICS OF INDUCTION MOTOR CONTROL}

Variable Frequency Drive (VFD) is commonly used for controlling the operating shaft speed and electromagnetic torque of an induction motor. There are different control schemes used in VFDs. Constant Volts/Hertz (V/f), Direct-Torque Control (DTC), and Field-Oriented Control (FOC) are the most popular control strategies. The simplest control method is the $\mathrm{V} / \mathrm{F}$ method where the ratio of voltage to frequency is kept constant. Due to its open-loop control method it suffers from poor dynamic response. For a better dynamic response, DTC or FOC are utilized in VFDs where instant electromagnetic torque can be obtained. The DTC method does not require machine parameters to implement and its drawback is that this control method has a higher torque ripple, whereas the FOC requires machine parameters, such as stator and rotor leakage inductance, however, the FOC has much lower torque ripple. In this work, FOC and V/f will be used as the primary control method. First, the mathematical model representation of a three-phase squirrel-cage induction motor is derived. At the end of this chapter, the basics of a three-phase voltage source inverter as well as gate signal generation for the switches and the effect of PWM signals will be reviewed. 


\subsection{Induction Motor Model}

In this section, the mathematical model of a three-phase induction motor is derived. The three phases of the motor will be denoted as $a, b$, and $c$. First, this model will be derived in the abc-coordinate, where each phase has a $120^{\circ}$ phase shift from each other. Then, this model will be transformed into a $q d 0$-reference frame using Park's transformation matrix [8].

\subsubsection{Induction motor model in the the $a b c$-reference frame}

Let us consider a three-phase squirrel cage induction motor with three stator windings $(\mathrm{a}, \mathrm{b}, \mathrm{c}$,$) as shown in Fig. 2.1. The mathematical model of this motor comprises three$ stator and three rotor voltage equations and a second-order mechanical equation of motion. The Induction machine's model is well-known and, for the sake of simplicity, this work will not include the preliminary equations or proofs. Readers are urged to refer to [8] for more details.

Using Krause's notation [8], the voltage equations are expressed in motor notation as the following set of six first-order differential equations,

$$
\begin{aligned}
& v_{a s}=R_{s} i_{a s}+\frac{d \lambda_{a s}}{d t} \\
& v_{b s}=R_{s} i_{b s}+\frac{d \lambda_{b s}}{d t} \\
& v_{c s}=R_{s} i_{c s}+\frac{d \lambda_{c s}}{d t} \\
& v_{a r}=R_{r} i_{a r}+\frac{d \lambda_{a r}}{d t} \\
& v_{b r}=R_{r} i_{b r}+\frac{d \lambda_{b r}}{d t} \\
& v_{c r}=R_{r} i_{c r}+\frac{d \lambda_{c r}}{d t}
\end{aligned}
$$


where variables $v, i, R$, and $\lambda$ represent, respectively, voltage, current, resistance, and magnetic flux of the induction motor and variables with the subscripts $s$ and $r$ also represent corresponding stator and rotor variables.

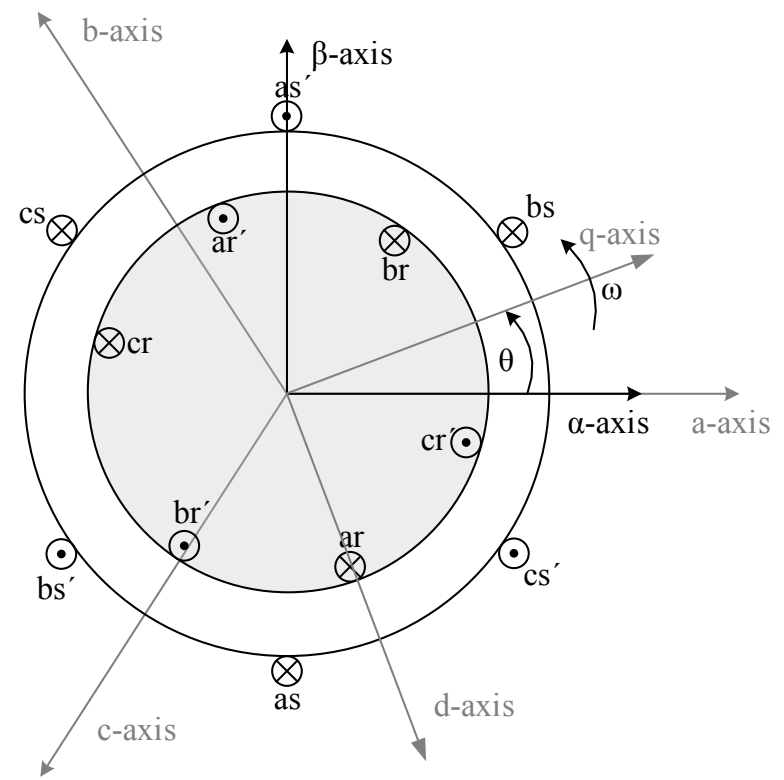

Figure 2.1: Squirrel-cage induction motor with three-phase $a b c$, arbitrary $(q d 0)$, and stationary $(\alpha \beta 0)$ reference frames.

Using a simpler notation are the following Equations (2.7) and (2.8)

$$
\begin{aligned}
\left(\mathbf{f}_{a b c s}\right)^{T} & =\left[\begin{array}{lll}
f_{a s} & f_{b s} & f_{c s}
\end{array}\right] \\
\left(\mathbf{f}_{a b c r}\right)^{T} & =\left[\begin{array}{lll}
f_{a r} & f_{b r} & f_{c r}
\end{array}\right]
\end{aligned}
$$

where $\mathbf{f}$ can be replaced with voltage or current, Equations (2.1) through (2.6) can be rewritten as

$$
\begin{aligned}
& \mathbf{v}_{a b c s}=\mathbf{r}_{s} \mathbf{i}_{a b c s}+p \boldsymbol{\lambda}_{a b c s} \\
& \mathbf{v}_{a b c r}=\mathbf{r}_{r} \mathbf{i}_{a b c r}+p \boldsymbol{\lambda}_{a b c r}
\end{aligned}
$$


where variables $\mathbf{v}$ and $\mathbf{i}$ are used for voltage and current respectively and the subscript of the $a b c$ is used for abc (three-phase reference frame) coordinate system. The parameters associated with the stator and the rotor are named $s$ and $r$, respectively. For a magnetically linear system, in a matrix form, the flux linkages are expressed as

$$
\left[\begin{array}{l}
\boldsymbol{\lambda}_{a b c s} \\
\boldsymbol{\lambda}_{a b c r}
\end{array}\right]=\left[\begin{array}{cc}
\mathbf{L}_{s} & \mathbf{L}_{s r} \\
\left(\mathbf{L}_{s r}\right)^{T} & \mathbf{L}_{r}
\end{array}\right]\left[\begin{array}{l}
\mathbf{i}_{a b c s} \\
\mathbf{i}_{a b c r}
\end{array}\right]
$$

where $\mathbf{L}_{s}, \mathbf{L}_{r}$, and $\mathbf{L}_{s r}$ are defiend as

$$
\begin{aligned}
\mathbf{L}_{s}= & {\left[\begin{array}{ccc}
L_{l s}+L_{m s} & -\frac{1}{2} L_{m s} & -\frac{1}{2} L_{m s} \\
-\frac{1}{2} L_{m s} & L_{l s}+L_{m s} & -\frac{1}{2} L_{m s} \\
-\frac{1}{2} L_{m s} & -\frac{1}{2} L_{m s} & L_{l s}+L_{m s}
\end{array}\right] } \\
\mathbf{L}_{r}= & {\left[\begin{array}{ccc}
L_{l r}+L_{m r} & -\frac{1}{2} L_{m r} & -\frac{1}{2} L_{m r} \\
-\frac{1}{2} L_{m r} & L_{l s}+L_{m r} & -\frac{1}{2} L_{m r} \\
-\frac{1}{2} L_{m r} & -\frac{1}{2} L_{m r} & L_{l r}+L_{m r}
\end{array}\right] } \\
\mathbf{L}_{s r}= & L_{s r}\left[\begin{array}{ccc}
\cos \theta_{r} & \cos \left(\theta_{r}+\frac{2 \pi}{3}\right) & \cos \left(\theta_{r}-\frac{2 \pi}{3}\right) \\
\cos \left(\theta_{r}-\frac{2 \pi}{3}\right) & \cos \theta_{r} & \cos \left(\theta_{r}+\frac{2 \pi}{3}\right) \\
\cos \left(\theta_{r}+\frac{2 \pi}{3}\right) & \cos \left(\theta_{r}-\frac{2 \pi}{3}\right) & \cos \theta_{r}
\end{array}\right] .
\end{aligned}
$$

In Equations (2.12) through (2.14), $L_{l s}$ and $L_{m s}$ are, respectively, the leakage and magnetizing inductances of the stator windings. $L_{l r}$ and $L_{m r}$ are, respectively, the leakage and magnetizing inductances of the rotor windings. $L_{s r}$ is the magnitude of the mutual inductances between stator and rotor windings. $\theta_{r}$ is the angle between the a-axis of the stator and the a-axis of the rotor. At this stage, rotor variables are referred to stator windings by using the suitable turns ratio. Therefore, the new 
equations for rotor voltage, current, and magnetic flux are expressed as:

$$
\begin{aligned}
\mathbf{i}_{a b c r}^{\prime} & =\frac{N_{r}}{N_{s}} \mathbf{i}_{a b c r} \\
\mathbf{v}_{a b c r}^{\prime} & =\frac{N_{r}}{N_{s}} \mathbf{v}_{a b c r} \\
\lambda_{a b c r}^{\prime} & =\frac{N_{r}}{N_{s}} \boldsymbol{\lambda}_{a b c r}
\end{aligned}
$$

where $N_{r}$ is the number of the turn ratio of the rotor and $N_{s}$ is the stator turn ratio. A prime is used to denote referred rotor parameters to the stator windings. In order to refer Equation (2.11) to the stator windings, first, $\mathbf{L}_{r}$ and $\mathbf{L}_{s r}$ in Equations (2.13) and (2.14) must be referred to the stator. Therefore, by defining $L_{m s}=\frac{N_{s}}{N_{r}} L_{s r}$, Equation (2.14) becomes

$$
\mathbf{L}_{s r}^{\prime}=\frac{N_{s}}{N_{r}} \mathbf{L}_{s r}=L_{m s}\left[\begin{array}{ccc}
\cos \theta_{r} & \cos \left(\theta_{r}+\frac{2 \pi}{3}\right) & \cos \left(\theta_{r}-\frac{2 \pi}{3}\right) \\
\cos \left(\theta_{r}-\frac{2 \pi}{3}\right) & \cos \theta_{r} & \cos \left(\theta_{r}+\frac{2 \pi}{3}\right) \\
\cos \left(\theta_{r}+\frac{2 \pi}{3}\right) & \cos \left(\theta_{r}-\frac{2 \pi}{3}\right) & \cos \theta_{r}
\end{array}\right]
$$

from Equations (1.5-44) and (1.5-47) in [8], $L_{m r}$ can be expressed as

$$
L_{m r}=\left(\frac{N_{r}}{N_{s}}\right)^{2} L_{m s}
$$

and if we let

$$
\mathbf{L}_{r}^{\prime}=\left(\frac{N_{r}}{N_{s}}\right)^{2} \mathbf{L}_{r}
$$

the rotor inductance matrix, Equation (2.13), becomes as given in 


$$
\mathbf{L}_{r}^{\prime}=\left[\begin{array}{ccc}
L_{l r}^{\prime}+L_{m s} & -\frac{1}{2} L_{m s} & -\frac{1}{2} L_{m s} \\
-\frac{1}{2} L_{m s} & L_{l r}+L_{m s} & -\frac{1}{2} L_{m s} \\
-\frac{1}{2} L_{m s} & -\frac{1}{2} L_{m s} & L_{l r}^{\prime}+L_{m s}
\end{array}\right]
$$

where

$$
L_{l r}^{\prime}=\left(\frac{N_{r}}{N_{s}}\right)^{2} L_{l r}
$$

Referring the rotor resistance to the stator side can be expressed as

$$
\mathbf{r}_{r}^{\prime}=\left(\frac{N_{r}}{N_{s}}\right)^{2} \mathbf{r}_{r}
$$

Ultimately, the final flux linkage matrix in Equation (2.11) referred to stator windings is

$$
\left[\begin{array}{l}
\boldsymbol{\lambda}_{a b c s} \\
\boldsymbol{\lambda}_{a b c r}^{\prime}
\end{array}\right]=\left[\begin{array}{cc}
\mathbf{L}_{s} & \mathbf{L}_{s r}^{\prime} \\
\left(\mathbf{L}_{s r}^{\prime}\right)^{T} & \mathbf{L}_{r}^{\prime}
\end{array}\right]\left[\begin{array}{l}
\mathbf{i}_{a b c s} \\
\mathbf{i}_{a b c r}^{\prime}
\end{array}\right]
$$

and using Equation (2.9) through (2.10) and (2.24), the newly defined rotor variables, the voltage equations become

$$
\left[\begin{array}{c}
\mathbf{v}_{a b c s} \\
\mathbf{v}_{a b c r}^{\prime}
\end{array}\right]=\left[\begin{array}{cc}
\mathbf{r}_{s}+p \mathbf{L}_{s} & p \mathbf{L}_{s r}^{\prime} \\
p\left(\mathbf{L}_{s r}^{\prime}\right)^{T} & \mathbf{r}_{r}^{\prime}+p \mathbf{L}_{r}
\end{array}\right]\left[\begin{array}{l}
\mathbf{i}_{a b c s} \\
\mathbf{i}_{a b c r}^{\prime}
\end{array}\right]
$$

where $p$ denotes $\frac{d}{d t}$. 


\subsubsection{Induction motor model in an arbitrary $q d 0$ reference frame}

The qd0 transformation is a mathematical transformation in which an electrical three-phase $a b c$ system is transformed to an arbitrary reference frame. The arbitrary reference frame can be changed depending upon where the new reference frame is placed. Motor inductances, for both stator and rotor in this reference frame, appear as constants, therefore, the analysis of this three-phase system is simplified. The transformation matrix was originally proposed by Park and Park's transformation, in which $\mathbf{K}_{s}$ is defined as

$$
\mathbf{K}_{s}=\frac{2}{3}\left[\begin{array}{ccc}
\cos \beta & \cos \left(\beta-\frac{2 \pi}{3}\right) & \cos \left(\beta+\frac{2 \pi}{3}\right) \\
\sin \beta & \sin \left(\beta-\frac{2 \pi}{3}\right) & \sin \left(\beta+\frac{2 \pi}{3}\right) \\
\frac{1}{2} & \frac{1}{2} & \frac{1}{2}
\end{array}\right]
$$

and the derivative of the inverse of Equation (2.26) is expressed as

$$
p\left[\mathbf{K}_{s}^{-1}\right]=\omega\left[\begin{array}{ccc}
-\sin \beta & \cos \beta & 0 \\
-\sin \left(\beta-\frac{2 \pi}{3}\right) & \cos \left(\beta-\frac{2 \pi}{3}\right) & 0 \\
-\sin \left(\beta+\frac{2 \pi}{3}\right) & \cos \left(\beta+\frac{2 \pi}{3}\right) & 0
\end{array}\right]
$$

where $\beta=\theta$ and $\theta$ is the angle between the $a$-axis and $q$-axis of the arbitrary $q d 0$ reference frame. Aligning the q-axis of the arbitrary reference frame with the q-axis of the rotor is called a rotor reference frame. In this reference frame, stator variables must be projected to the rotor axis. For transforming rotor parameters to stator or vice verse, the slip angle (difference angle between rotor and stator position) is used. Therefore, using Park's transformation to transform rotor variables to an arbitrary reference frame will be called $\mathbf{K}_{r}$ with $\beta=\theta-\theta_{r}$ where $\theta_{r}$ is the angle between 
rotor the a-axis and the q-axis of the arbitrary reference frame. In the case of a rotor reference frame, $\theta_{r}=0$. Now, it is possible to transform Equations (2.24) and (2.25) from the $a b c$-coordinate system into the $q d 0$-coordinate system. Therefore, by defining

$$
\mathbf{f}_{q d 0}=\mathbf{K}_{s} \mathbf{f}_{a b c}
$$

and applying Equation (2.28) into Equations (2.24) and (2.25), for stator parameters, yields Equation (2.31)

$$
\begin{aligned}
\mathbf{K}_{s}^{-1} \mathbf{v}_{q d 0 s} & =\mathbf{r}_{s} \mathbf{K}_{s}^{-1} \mathbf{i}_{q d 0 s}+p\left[\mathbf{K}_{s}^{-1} \boldsymbol{\lambda}_{q d 0 s}\right] \\
\mathbf{K}_{s}^{-1} \mathbf{v}_{q d 0 s}= & \mathbf{r}_{s} \mathbf{K}_{s}^{-1} \mathbf{i}_{q d 0 s}+\omega p\left[\mathbf{K}_{s}^{-1}\right] \boldsymbol{\lambda}_{q d 0 s}+\mathbf{K}_{s}^{-1} \frac{d}{d t} \boldsymbol{\lambda}_{q d 0 s} \\
\mathbf{v}_{q d 0 s} & =\mathbf{r}_{s} \mathbf{i}_{q d 0 s}+\omega\left[\begin{array}{ccc}
0 & 1 & 0 \\
-1 & 0 & 0 \\
0 & 0 & 0
\end{array}\right] \boldsymbol{\lambda}_{q d 0 s}+\frac{d}{d t} \boldsymbol{\lambda}_{q d 0 s}
\end{aligned}
$$

$\beta=\theta-\theta_{r}$. In a similar fashion, as Equations (2.29) - (2.31), using $\beta=\theta-\theta_{r}$, the rotor voltage equations become

$$
\mathbf{v}_{q d 0 r}^{\prime}=\mathbf{r}_{r}^{\prime} \mathbf{i}_{q d 0 r}^{\prime}+\left(\omega-\omega_{r}\right)\left[\begin{array}{ccc}
0 & 1 & 0 \\
-1 & 0 & 0 \\
0 & 0 & 0
\end{array}\right] \boldsymbol{\lambda}_{q d 0 r}^{\prime}+\frac{d}{d t} \boldsymbol{\lambda}_{q d 0 r}^{\prime}
$$

In order to derive the stator and rotor equations for the stationary reference frame $\omega=0$, for the reference frame fixed in the rotor, $\omega=\omega_{r}$, and $\omega=\omega_{s}$ is used for the synchronously rotating reference frame. Putting the Equations in a matrix form, we 
have

$$
\left[\begin{array}{c}
v_{q s} \\
v_{d s} \\
v_{0 s} \\
v_{q r} \\
v_{d r} \\
v_{0 r}
\end{array}\right]=\left[\begin{array}{cccccc}
r_{s} & 0 & 0 & 0 & 0 & 0 \\
0 & r_{s} & 0 & 0 & 0 & 0 \\
0 & 0 & r_{s} & 0 & 0 & 0 \\
0 & 0 & 0 & r_{r}^{\prime} & 0 & 0 \\
0 & 0 & 0 & 0 & r_{r}^{\prime} & 0 \\
0 & 0 & 0 & 0 & 0 & r_{r}^{\prime}
\end{array}\right]\left[\begin{array}{c}
i_{q s} \\
i_{d s} \\
i_{0 s} \\
i_{q r}^{\prime} \\
i_{d r}^{\prime} \\
i_{0 r}^{\prime}
\end{array}\right]+\left[\begin{array}{cccccc}
0 & \omega & 0 & 0 & 0 & 0 \\
-\omega & 0 & 0 & 0 & 0 & 0 \\
0 & 0 & 0 & 0 & 0 & 0 \\
0 & 0 & 0 & 0 & \left(\omega-\omega_{r}\right) & 0 \\
0 & 0 & 0 & -\left(\omega-\omega_{r}\right) & 0 & 0 \\
0 & 0 & 0 & 0 & 0 & 0
\end{array}\right]\left[\begin{array}{c}
\lambda_{q s} \\
\lambda_{d s} \\
\lambda_{0 s} \\
\lambda_{q r}^{\prime} \\
\lambda_{d r}^{\prime} \\
\lambda_{0 r}^{\prime}
\end{array}\right]+\frac{d}{d t}\left[\begin{array}{c}
\lambda_{q s} \\
\lambda_{d s} \\
\lambda_{0 s} \\
\lambda_{q r}^{\prime} \\
\lambda_{d r}^{\prime} \\
\lambda_{0 r}^{\prime}
\end{array}\right]
$$

or the expanded form of the matrix above is written as

$$
\begin{aligned}
& v_{q s}=r_{s} i_{s}+\omega \lambda_{d s}+\frac{d}{d t} \lambda_{q s} \\
& v_{d s}=r_{s}-\omega \lambda_{q s}+\frac{d}{d t} \lambda_{d s} \\
& v_{0 s}=r_{s}+\frac{d}{d t} \lambda_{0 s} \\
& v_{q r}^{\prime}=r_{r}^{\prime}+\left(\omega-\omega_{r}\right) \lambda_{d r}^{\prime}+\frac{d}{d t} \lambda_{q r}^{\prime} \\
& v_{d r}^{\prime}=r_{r}^{\prime}-\left(\omega-\omega_{r}\right) \lambda_{q r}^{\prime}+\frac{d}{d t} \lambda_{d r}^{\prime} \\
& v_{0 r}^{\prime}=r_{r}^{\prime}+\frac{d}{d t} \lambda_{0 r}^{\prime}
\end{aligned}
$$

Due to the rotor short-circuit winding of a squirrel cage induction motor, values of $v_{q r}^{\prime}$ and $v_{d r}^{\prime}$ are equal to zero. The transformed flux linkage equations for a magnetically linear system are

$$
\left[\begin{array}{l}
\boldsymbol{\lambda}_{q d 0 s} \\
\boldsymbol{\lambda}_{q d 0 r}^{\prime}
\end{array}\right]=\left[\begin{array}{cc}
\mathbf{K}_{s} \mathbf{L}_{s} \mathbf{K}_{s}^{-1} & \mathbf{K}_{s} \mathbf{L}_{s r}^{\prime} \mathbf{K}_{r}^{-1} \\
\mathbf{K}_{r}\left(\mathbf{L}_{s r}^{\prime}\right)^{T} \mathbf{K}_{s}^{-1} & \mathbf{K}_{r} \mathbf{L}_{r}^{\prime} \mathbf{K}_{r}^{-1}
\end{array}\right]\left[\begin{array}{l}
\mathbf{i}_{q d 0 s} \\
\mathbf{i}_{q d 0 r}^{\prime}
\end{array}\right]
$$

Performing the transformation in the equation above, the current-flux relationship in the $q d 0$ reference frame is expressed as 


$$
\left[\begin{array}{c}
\lambda_{q s} \\
\lambda_{d s} \\
\lambda_{0 s} \\
\lambda_{q r}^{\prime} \\
\lambda_{d r}^{\prime} \\
\lambda_{0 r}^{\prime}
\end{array}\right]=\left[\begin{array}{cccccc}
L_{l s}+L_{m} & 0 & 0 & L_{m} & 0 & 0 \\
0 & L_{l s}+L_{m} & 0 & 0 & L_{m} & 0 \\
0 & 0 & L_{l s}+L_{m} & 0 & 0 & 0 \\
L_{m} & 0 & 0 & L_{l r}^{\prime}+L_{m} & 0 & 0 \\
0 & L_{m} & 0 & 0 & L_{l r}^{\prime}+L_{m} & 0 \\
0 & 0 & 0 & 0 & 0 & L_{l r}^{\prime}
\end{array}\right]\left[\begin{array}{c}
i_{q s} \\
i_{d s} \\
i_{0 s} \\
i_{q r}^{\prime} \\
i_{d r}^{\prime} \\
i_{0 r}^{\prime}
\end{array}\right]
$$

and in equation form

$$
\begin{aligned}
& \lambda_{q s}=\left(L_{l s}+L_{m}\right) i_{q s}+L_{m} i_{q r} \\
& \lambda_{d s}=\left(L_{l s}+L_{m}\right) i_{d s}+L_{m} i_{d r} \\
& \lambda_{0 s}=L_{l s} i_{0 s} \\
& \lambda_{q r}^{\prime}=L_{m} i_{q s}+\left(L_{l r}^{\prime}+L_{m}\right) i_{q r}^{\prime} \\
& \lambda_{d r}^{\prime}=\left(L_{l r}^{\prime}+L_{m}\right) i_{d r}^{\prime}+L_{m} i_{d s} \\
& \lambda_{0 r}^{\prime}=L_{l r}^{\prime} i_{0 r}^{\prime}
\end{aligned}
$$

The stator and rotor voltage in Equations (2.34) - (2.39) and the stator and rotor fluxcurrent relationship in Equations (2.42) and (2.47) are the final induction motor model in the $q d 0$-coordinate system. These equations are used to derive the electromagnetic torque equation.

\subsection{Induction Motor Torque Equation}

In this section, the dynamic equations of an induction motor are used to develop an equation for electromagnetic torque. By assuming the linear characteristics of the 
magnetic material of the core of an induction motor, the windings of the motor can be represented as mutually coupled. Using the energy conversion principles developed in [8], the electromagnetic torque of an induction motor can be obtained by the following formula

$$
T_{e}=\left(\frac{P}{2}\right)\left(\mathbf{i}_{a b c s}\right)^{T} \frac{\partial}{\partial \theta_{r}}\left[\mathbf{L}_{s r}^{\prime}\right] \mathbf{i}_{a b c r}^{\prime}
$$

and, by performing Park's transformation, the electromagnetic torque in the $q d 0$ coordinate is

$$
T_{e}=\left(\frac{P}{2}\right)\left(\left(\mathbf{K}_{p}\right)^{-1} \mathbf{i}_{q d 0 s}\right)^{T} \frac{\partial}{\partial \theta_{r}}\left[\mathbf{L}_{s r}^{\prime}\right]\left(\mathbf{K}_{r}\right)^{-1} \mathbf{i}_{q d 0 r}^{\prime}
$$

using flux-current relationships found in Equations (2.42) - (2.47). The electromagnetic torque expression yields the following equation and is expressed in terms of currents as

$$
T_{e}=\left(\frac{3}{2}\right)\left(\frac{P}{2}\right) L_{m}\left(i_{q s} i_{d r}^{\prime}-i_{d s} i_{q r}^{\prime}\right)
$$

and other equivalent expressions in terms of stator and rotor flux are given as

$$
\begin{aligned}
& T_{e}=\left(\frac{3}{2}\right)\left(\frac{P}{2}\right)\left(\lambda_{q r}^{\prime} i_{d r}^{\prime}-\lambda_{d r}^{\prime} i_{q r}^{\prime}\right) \\
& T_{e}=\left(\frac{3}{2}\right)\left(\frac{P}{2}\right)\left(\lambda_{d s} i_{q s}-\lambda_{q s} i_{d s}\right)
\end{aligned}
$$

The electromagnetic torque in Equation 2.51 will be used in the FOC to produce the electromagnetic torque command from the measured current. 


\subsection{Field-Oriented Control Method}

The FOC method is a common technique to control an induction motor. This control method has a high dynamic response and is commonly utilized in industry. The FieldOriented Control method of controlling an induction motor enables the behaviour of a separately excited DC motor. In many motor drive systems, it is preferable to make the drive act as a torque controller in which the electromagnetic torque produced by the motor could almost instantaneously be made equal to a torque command by the FOC. Equation (2.51) may be expressed as

$$
T_{e}=-\frac{3}{2} \frac{P}{2}\left|\lambda_{q d r}^{\prime}\right|\left|i_{q d r}^{\prime}\right| \sin \theta
$$

where, for any $\lambda_{q d r}^{\prime}$ and $i_{q d r}^{\prime}$, electromagnetic torque is maximized when $\theta=90^{\circ}$ or the rotor flux linkage and the rotor current vectors are perpendicular. Therefore, if we keep $\lambda_{q r}^{\prime}=0$ and $i_{d r}^{\prime}=0$, the rotor flux linkage and rotor current vectors are perpendicular. With new q-axis flux linkage and d-axis current equal to zero, the fluxcurrent relationship in Equations (2.42) - (2.47), without zero-sequence components, become

$$
\left[\begin{array}{c}
\lambda_{q s} \\
\lambda_{d s} \\
0 \\
\lambda_{d r}^{\prime}
\end{array}\right]=\left[\begin{array}{cccc}
L_{l s}+L_{m} & 0 & L_{m} & 0 \\
0 & L_{l s}+L_{m} & 0 & L_{m} \\
L_{m} & 0 & L_{l r}^{\prime}+L_{m} & 0 \\
0 & L_{m} & 0 & L_{l r}^{\prime}+L_{m}
\end{array}\right]\left[\begin{array}{c}
i_{q s} \\
i_{d s} \\
i_{q r}^{\prime} \\
0
\end{array}\right]
$$

therefore, the magnitude of the electromagnetic torque in Equation (2.51) may be expressed as 


$$
T_{e}=-\frac{3}{2} \frac{P}{2} \lambda_{d r}^{\prime} i_{q r}^{\prime}
$$

where, from the equations in (2.54), $i_{q r}^{\prime}=-\frac{L_{m}}{L_{r r}} i_{q s}$ and $\lambda_{d r}^{\prime}=L_{m} i_{d s}$. The values of $i_{q s}$ and $i_{d s}$ are obtained using Park's transformation of the measured currents in the $a b c$ frame. The torque equation can also be written by two parameters of the $q$-axis stator current and the $d$-axis rotor flux as

$$
T_{e}=\frac{3}{2} \frac{P}{2} \frac{L_{m}}{L_{r r}} \lambda_{d r}^{\prime} i_{q s}
$$

By knowing the relationship between the electromagnetic torque and the $q$-axis, the $i_{q s}^{*}$ required to produce the electromagnetic torque by the motor can be calculated. Re-writing 2.56 yields

$$
i_{q s}^{*}=\frac{2}{3} \frac{2}{P} \frac{L_{r r}}{L_{m}} \frac{T_{e}^{*}}{\lambda_{d r}^{\prime}}
$$

where the superscript $*$ represents the reference values in the FOC. Figure 2.2 illustrates the whole FOC algorithm implementation used to control the induction motor. In this design, the $i_{q s}^{*}$ is defined by Equation (2.57). The difference between the rated rotor flux, $\lambda_{d r}^{*}$, and the actual produced rotor flux, $\lambda_{d r}$, of the motor is fed to a proportional-integral (PI) controller. This PI controller's output divided by $\hat{L}_{m}$ yields us the stator d-axis current $i_{d s}^{*}$. The produced rotor flux also can be obtained from the $i_{d s}$ and this value gets compared to the rated flux of the motor. The rated flux can be obtained as following

$$
\lambda_{d r, \text { rated }}=L_{m} I_{m}=L_{m} \frac{V_{p h, p e a k}}{X_{m}}=\frac{V_{p h, p e a k}}{\omega_{b}}
$$


The error between the measured the $d$-axis current, $i_{d s}$, and d-axis reference current,

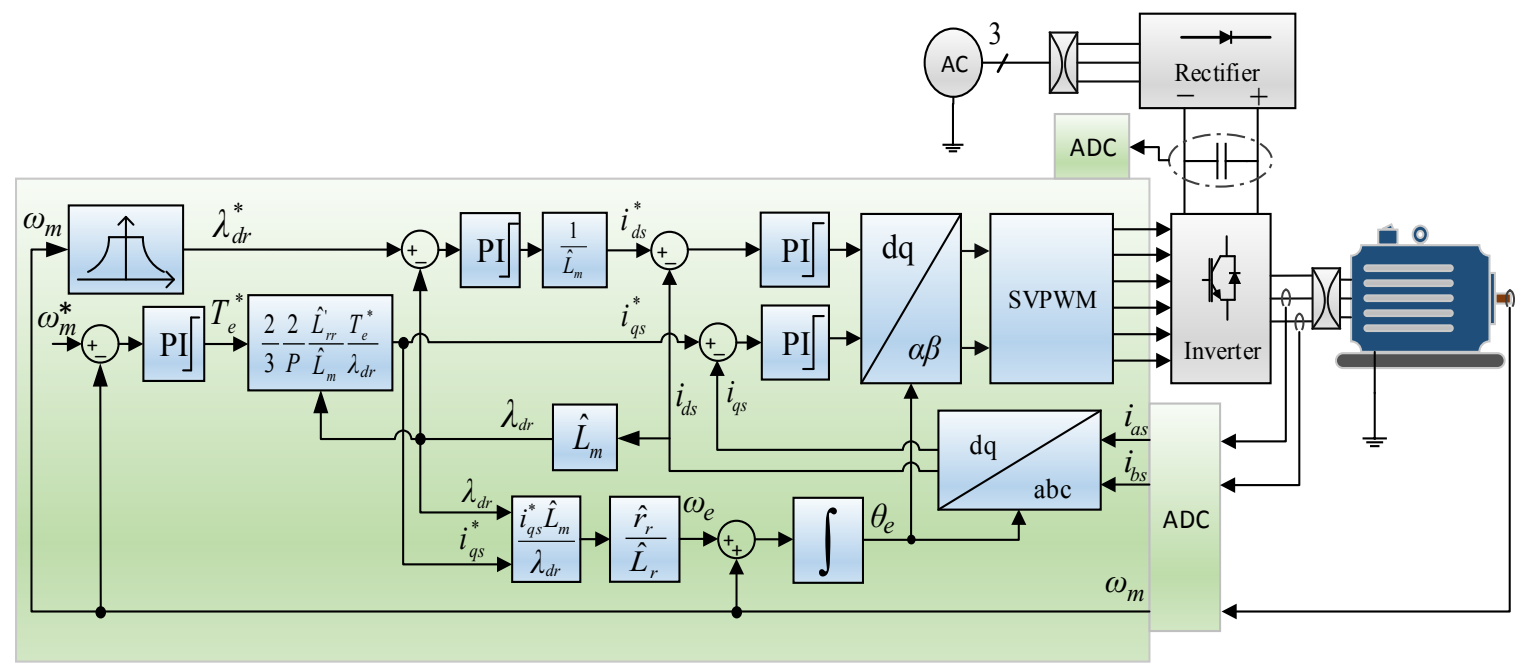

Figure 2.2: Schematic of the field-oriented control with Space-Vector PWM

$i_{d s}^{*}$, will be fed to the PI controller to convert this error to the $d$-axis voltage reference,

$v_{d s}^{*}$. The same procedure is applied to the $q$-axis current, $i_{q s}$, to obtain the $q$-axis voltage reference, $v_{q s}^{*}$. These voltages in the rotating $q d 0$ reference frame, will be transformed into the stationary reference frame $(\alpha \beta 0)$. In the next section, this transformation will be discussed in more detail. The $v_{\alpha}$ and $v_{\beta}$ is fed to a Space-Vector Pulse Width Modulation (SVPWM) block to generate the gating signals for the threephase voltage source inverter. Using SVPWM gives a higher DC-link utilization of the inverter and has a lower total harmonic distortion (THD).

\subsection{SVPWM Background}

Many comprehensive analyses have shown the relationship between Carrier-Based PWM and SVPWM $[9,10]$. The SVPWM techniques have advantages in terms of control flexibility, low harmonics, and better dynamic performance [11]. Furthermore, 


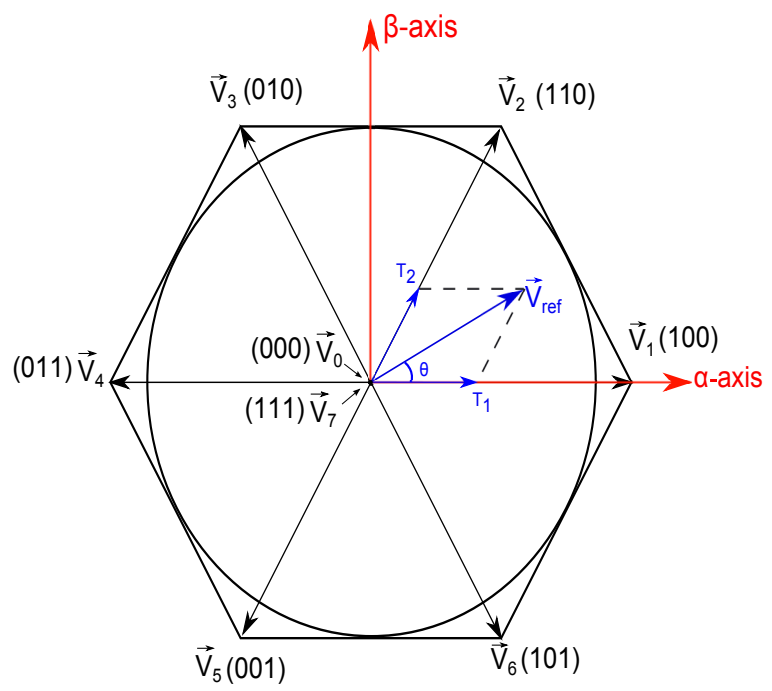

Figure 2.3: Space-vector diagram for a two-level voltage inverter

these real-time modulation techniques are widely used in voltage-source inverters. In this section, SVPWM is briefly reviewed.

\subsubsection{Angle and Voltage Reference Vector}

Assume that a three-phase AC motor is driven by an ideal three-phase AC voltage source expressed as the following space vector

$$
\vec{v}^{*}=\frac{2}{3}\left(v_{a}^{*}+v_{b}^{*} e^{j \frac{2 \pi}{3}}+v_{c}^{*} e^{-j \frac{2 \pi}{3}}\right) .
$$

This space vector can also be represented in the stationary reference frame with two orthogonal axes $(\alpha$ and $\beta$ ). The $\alpha$-axis is aligned with the horizontal direction and a-axis and the $\beta$-axis is in the vertical direction, as shown in Figure 2.3. Hence, the parameters in the $a b c$-reference frame can be transformed into the stationary reference frame, $\alpha \beta 0$, using Clark's transformation [8] 


$$
\mathbf{f}_{\alpha \beta 0}=[\mathbf{C}] \mathbf{f}_{a b c s}
$$

where $\mathbf{C}$ is

$$
\mathbf{C}=\frac{2}{3}\left[\begin{array}{ccc}
1 & -\frac{1}{2} & -\frac{1}{2} \\
0 & \frac{\sqrt{3}}{2} & -\frac{\sqrt{3}}{2} \\
\frac{1}{2} & \frac{1}{2} & \frac{1}{2}
\end{array}\right]
$$

and, we have

$$
\mathbf{v}_{\alpha \beta 0}=[\mathbf{C}] \mathbf{v}_{a b c s}
$$

using Equation (2.28),

$$
\begin{aligned}
\mathbf{v}_{\alpha \beta 0} & =[\mathbf{C}]\left[\mathbf{K}_{s}^{-1}\right] \mathbf{v}_{q d 0} \\
= & \frac{2}{3}\left[\begin{array}{ccc}
1 & -\frac{1}{2} & -\frac{1}{2} \\
0 & \frac{\sqrt{3}}{2} & -\frac{\sqrt{3}}{2} \\
\frac{1}{2} & \frac{1}{2} & \frac{1}{2}
\end{array}\right]\left[\begin{array}{ccc}
\cos (\theta) & \sin (\theta) & 1 \\
\cos \left(\theta-\frac{2 \pi}{3}\right) & \sin \left(\theta-\frac{2 \pi}{3}\right) & 1 \\
\cos \left(\theta+\frac{2 \pi}{3}\right) & \sin \left(\theta+\frac{2 \pi}{3}\right) & 1
\end{array}\right] \mathbf{v}_{q d 0} \\
= & {\left[\begin{array}{ccc}
\cos (\theta) & \sin (\theta) & 0 \\
-\sin (\theta) & \cos (\theta) & 0 \\
0 & 0 & 1
\end{array}\right] \mathbf{v}_{q d 0} }
\end{aligned}
$$

The matrix above is used for transforming the $q d 0$ variables to $\alpha \beta 0$ stationary reference frame. The three-phase current in a healthy three-phase motor is shown in Fig. 2.4a. Using Equation (2.62), the current in $\alpha \beta 0$-coordinate can be obtained. The currents in $\alpha \beta 0$-coordinate are shown in Fig. 2.4b. The $\alpha$-axis current on the 
$\mathrm{x}$-axis and $\beta$-axis current on the $\mathrm{y}$-axis will construct a circle in a healthy three-phase system as shown in Fig. 2.4c.

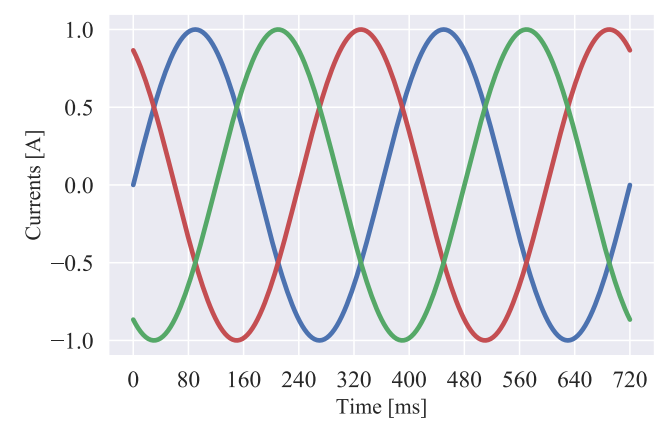

(a) $a b c$-coordinate

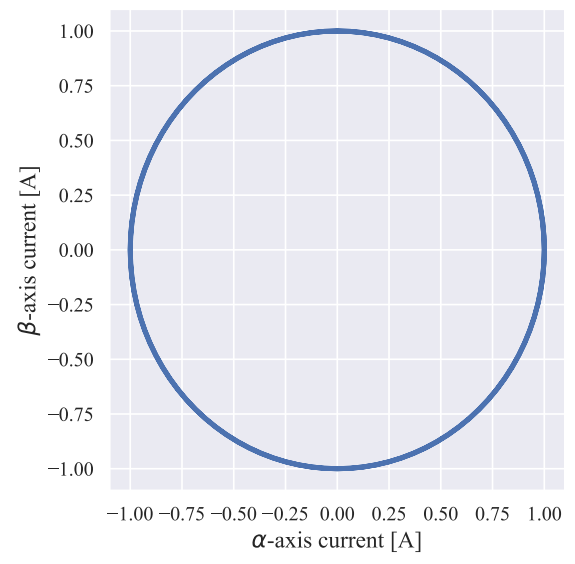

(c) $\alpha$ and $\beta$ axis

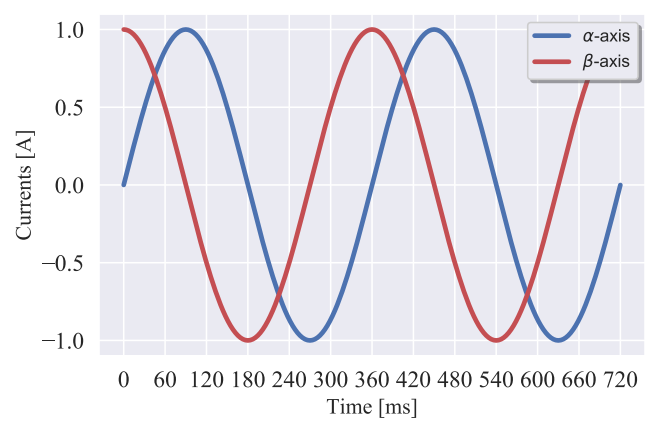

(b) $\alpha \beta 0$-coordinate

Figure 2.4: Illustration of three-phase current in $a b c$ and $\alpha \beta 0$-coordinates

The magnitude and the phase angle of the reference voltage vector are evaluated as

$$
\begin{aligned}
\left\|\vec{v}^{*}\right\| & =\sqrt{\left(v_{\alpha}^{*}\right)^{2}+\left(v_{\beta}^{*}\right)^{2}} \\
\theta & =\tan ^{-1} \frac{v_{\beta}^{*}}{v_{\alpha}^{*}}
\end{aligned}
$$


The magnitude of the desired output voltage, $\vec{V}_{\text {ref }}$, is calculated from the FOC design as an input to the SVPWM design. The desired $\theta$ is calculated using 2.68.

\subsubsection{Modulation Index}

The Fourier series expansion of a symmetrical wave voltage with a peak magnitude of $V_{d c} / 2$ has a fundamental with a magnitude $V_{\max }=2 V_{d c} / \pi$. The modulation index is defined as the ratio of the magnitude of the output voltage generated by the SVPWM to the fundamental peak value of the maximum square wave. Thus, the maximum modulation index of the SVPWM technique is

$$
m_{i}=\frac{\left\|\vec{V}_{\max }^{*}\right\|}{V_{\max }}=\frac{\frac{V_{d c}}{\sqrt{3}}}{\frac{2 V_{d c}}{\pi}}=0.907
$$

The $m_{i}$ will help differentiate the modulation index from the desired voltage reference, $\vec{V}^{*}$, input range.

\subsubsection{Time Calculations}

As shown in Fig. 2.3, $T_{1}$ and $T_{2}$ are the dwell times for vector $T_{1}$ and vector $T_{2}$. The dwell times for the $\alpha$ - and $\beta$-vectors essentially represent the on-time durations of the respective PWM signals during a sampling time. Assuming that the sampling period $T_{s}$ is sufficiently small, the duration times $T_{1}$ and $T_{2}$ of the $\alpha$ - and $\beta$-component vectors can be calculated as

$$
T_{1}=T_{s} a \frac{\sin \left(\frac{\pi}{3}-\theta\right)}{\sin \left(\frac{\pi}{3}\right)}, \quad T_{2}=T_{s} a \frac{\sin (\theta)}{\sin \left(\frac{\pi}{3}\right)}
$$

where $T_{s}$ is the sampling time and $a=\left\|\vec{v}^{*}\right\| /\left(2 V_{d c} / 3\right)$. Taking into consideration the maximum value of $\vec{v}^{*}$ and rearranging the above equations 


$$
\begin{aligned}
& T_{1}=T_{s} \sqrt{3} m_{a} \sin \left(\frac{\pi}{3}-\theta\right) \\
& T_{2}=T_{s} \sqrt{3} m_{a} \sin (\theta) \\
& T_{0}=T_{s}-T_{1}-T_{2}
\end{aligned}
$$

where $m_{a}=\vec{v}^{*} / V_{d c}$ lies in the range of $[0, \sqrt{3} / 3]$. Figure 2.5 illustrates the SVPWM output signals with their corresponding vectors for one period.

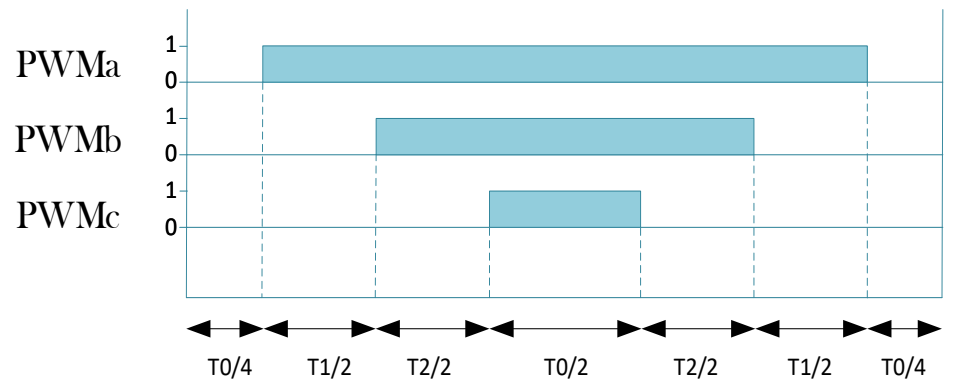

Figure 2.5: Space-vector PWM signals

Figure 2.6 shows a block diagram and signals for the time calculations using (2.71)-(2.73). The two inputs $m_{a}$ and $\theta$, provide the desired magnitude and frequency of the SVPWM output, while $T_{s}$ is the input sampling frequency. The Finite State Machine (FSM) module is used to generate PWM signals according to the times calculated in (2.71)-(2.73).

Now that the functionality of each module in the FOC method, as shown in Fig. 2.2, has been discussed, the following section will provide the FOC simulation results. The PWM signals for a specific duty cycle are generated from the finite-state machine block. Using a low-pass filter, the averaged PWM signals can be illustrated. The three filtered SVPWM signals for the voltage source inverter are shown in Fig. 2.7. 


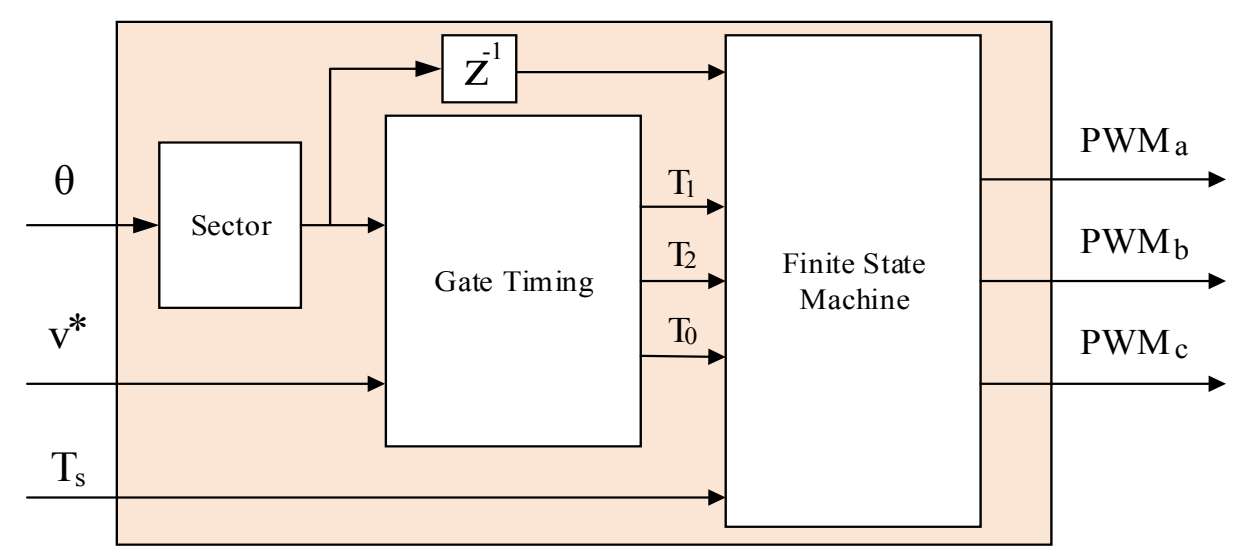

Figure 2.6: Space-vector PWM schematic for calculation of $T_{0}, T_{1}$, and $T_{2}$

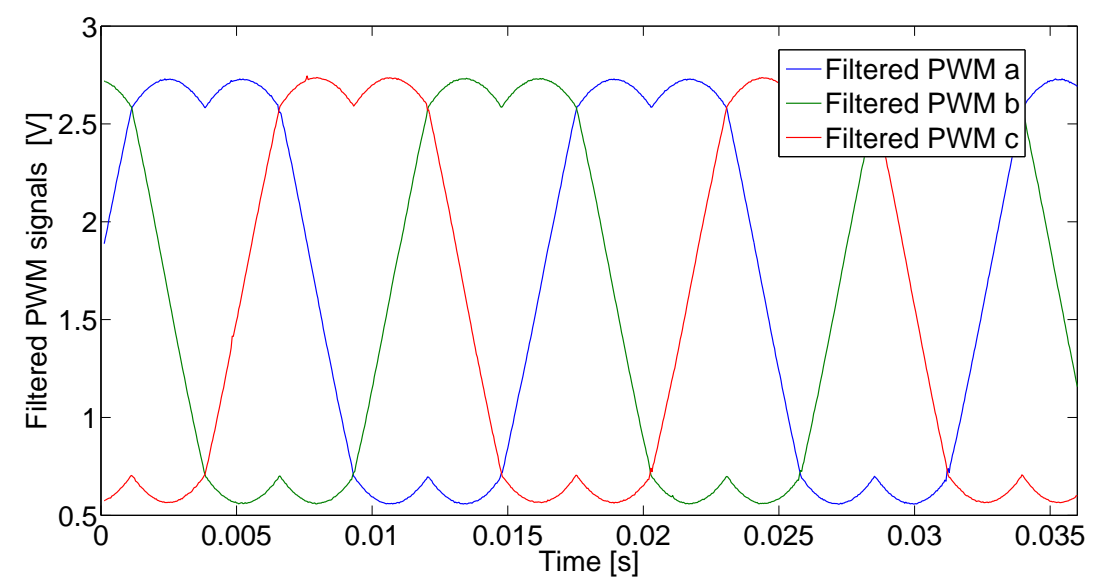

Figure 2.7: SVPWM signals for three-phase voltage source inverter

\subsection{Basic Operation of a Three-Phase Inverter}

In this section, the basic operation of the three-phase inverter and the fundamentals of the common-mode voltage are reviewed. Three-phase inverters consist of three single leg inverters. Each leg consists of two switches and each switch has a anti-parallel diode, as shown in Fig. 2.8.

The voltage source inverter cannot produce a pure sine wave. The PWM signals are applied to the gate-source of the transistor in order to turn $\mathrm{ON}$ and OFF the 


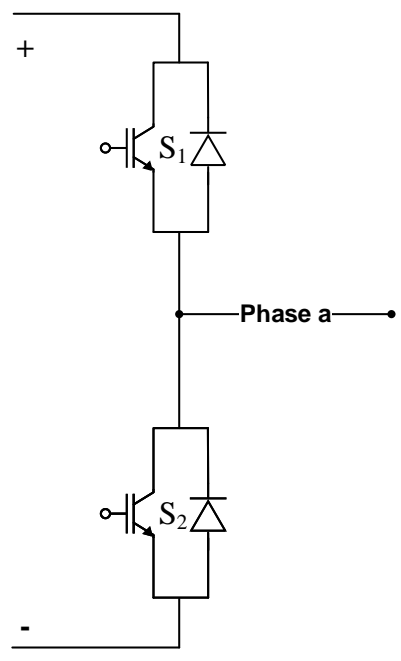

Figure 2.8: The schematic of one leg of the three-phase inverter.

switch. When $S_{1}$ is ON, phase $a$ will have $+V_{D C}$ and when $S_{2}$ is ON, the output voltage of phase $a$ will be zero. If both switches are kept ON, at the same time, the DC-link is shorted. Therefore, dead-time exists where both switches become OFF before turning the other switch ON. When both switches are OFF, the current in phase $a$ has to be able to conduct from either the positive DC-link or from the negative side of the DC-link.

To mitigate this problem, the anti-parallel diodes exist in the switches so that these diodes will allow the current to flow to the DC-link, as shown in Fig. 2.9.

\subsubsection{Common-Mode Voltage in Two-Level Inverter}

There are multiple techniques to generate PWM signals. Some techniques are discontinuous pulse width modulation (DPWM) and some continuous techniques such as sinusoidal PWM (SPWM) and space-vector pulse width modulation (SVPWM) $[12,13]$. The three-phase inverter can generate three pulsed sine waves with a $120^{\circ}$ phase shift. 

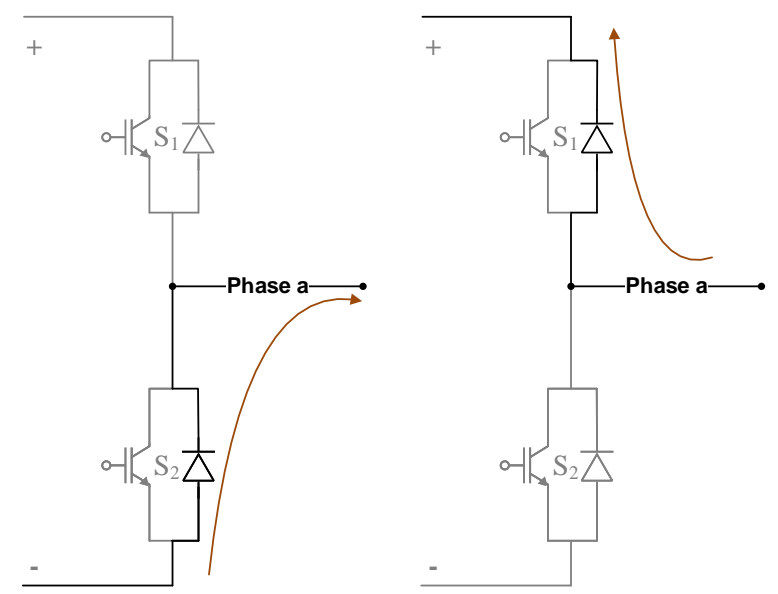

Figure 2.9: Operation of dead-time in one leg of the inverter for both positive and negative current.

Each type of PWM technique, due to the pulsed nature, causes inverters to apply discrete output voltages to the system, which produces undesired common-mode voltage. The definition of common mode voltage is as follows:

$$
V_{n o}=V_{C M V}=\frac{\left(V_{a n}+V_{b n}+V_{c n}\right)}{3}-V_{o}
$$

where $V_{a n}, V_{b n}$, and $V_{c n}$ are the voltage measured from the terminal to the ground of each phase and $V_{o}$ is $\frac{V_{d c}}{2}$, as shown in Fig. 2.10. In Chapter 3, more details about the effect of common-mode voltage and how to reduce it are provided. The Common-Mode Voltage (CMV) is never zero and its value is dependent on the status of the switches. However, in this section, a brief introduction of the CMV is presented. The CMVs are unique and their magnitude at a given time are dependent on the type of the PWM used in the design. The CMV value for SVPWM and SPWM are different.

The instantaneous common-mode voltage for a SVPWM can be calculated as: 


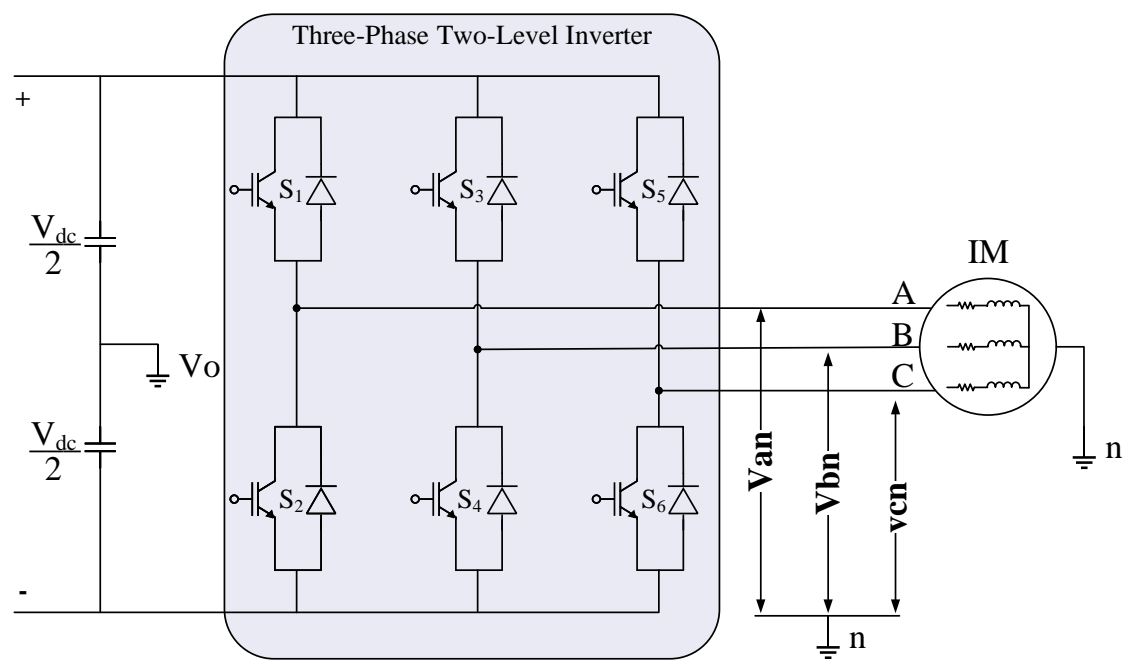

Figure 2.10: Common-mode voltage signals in a three-phase inverter

$$
V_{C M V}=\frac{1}{3}\left(S_{A} V_{a n}+S_{B} V_{b n}+S_{C} V_{c n}\right)
$$

For $\vec{V}_{1}$ as shown in Fig. 2.3

$$
\begin{aligned}
V_{C M V} & =\frac{1}{3}\left(S_{A} V_{a n}+S_{B} V_{b n}+S_{C} V_{c n}\right)-\frac{V_{d c}}{2} \\
& =\frac{1}{3}\left(1 \times \frac{V_{d c}}{2}+0 \times \frac{V_{d c}}{2}+0 \times \frac{V_{d c}}{2}\right)-\frac{V_{d c}}{2}=\frac{V_{d c}}{6}
\end{aligned}
$$

and for all vectors, $\vec{V}_{0}$ through $\vec{V}_{7}$, Table 2.1 presents the corresponding vector's $V_{C M V}$. Figure 2.11 presents the common-mode voltage for a SVPWM period. The $V_{C M V}$ is within $\pm \frac{V_{d c}}{2}$ and for a small values of $T_{1}$ and $T_{2}$, the $V_{C M V}$ can be changed from $-\frac{V_{d c}}{2}$ to $\frac{V_{d c}}{2}$. This sudden voltage change is undesirable and can create common-mode current spikes. 
Table 2.1: Common-mode voltage for all vectors in the SVPWM

\begin{tabular}{|c|c|c|}
\hline Vector & Switch Status & $V_{C M V}$ \\
\hline$\vec{V}_{1}$ & $(100)$ & $-\frac{V_{d c}}{6}$ \\
\hline$\vec{V}_{2}$ & $(110)$ & $\frac{V_{d c}}{6}$ \\
\hline$\vec{V}_{3}$ & $(010)$ & $-\frac{V_{d c}}{6}$ \\
\hline$\vec{V}_{4}$ & $(011)$ & $\frac{V_{d c}}{6}$ \\
\hline$\vec{V}_{5}$ & $(001)$ & $-\frac{V_{d c}}{6}$ \\
\hline$\vec{V}_{6}$ & $(101)$ & $\frac{V_{d c}}{6}$ \\
\hline$\vec{V}_{7}$ & $(111)$ & $\frac{V_{d c}}{2}$ \\
\hline$\vec{V}_{0}$ & $(000)$ & $-\frac{V_{d c}}{2}$ \\
\hline
\end{tabular}

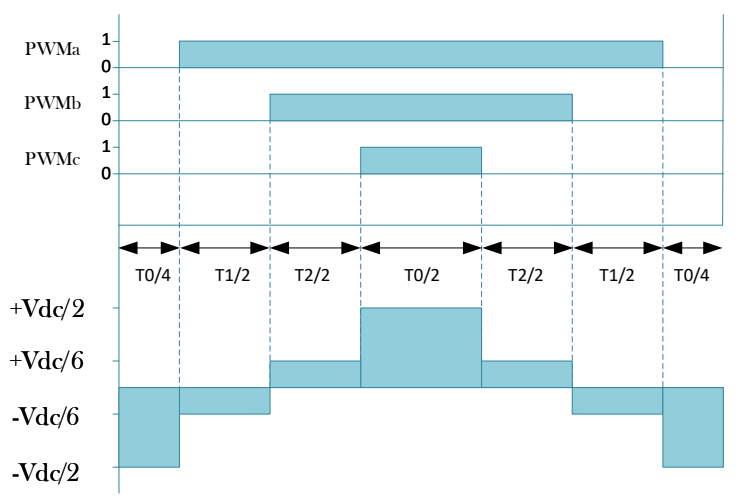

Figure 2.11: The common-mode voltage in SVPWM

\subsection{Field-Oriented Control Simulation}

In this section, Figure 2.12 presents results obtained from simulation of the FOC, inverter, and the motor model. The machine and control parameters are listed in Appendix A. The speed reference and speed response are shown in Fig. 2.12. The speed starts from zero and ramps to $200 \mathrm{rpm}$ and at $t=1 \mathrm{~s}$ ramps down to $-200 \mathrm{rpm}$. As shown in Fig. 2.12, multiple loads have been applied at different operating speeds of the motor while keeping the desired speed constant. The modulation technique of SVPWM has been used. In this simulation, no fault was introduced, and it was only 
run to verify that the simulation produces the expected results. This simulation will be used in Chapter 3 and Chapter 4 for both algorithm functionality and response verification.
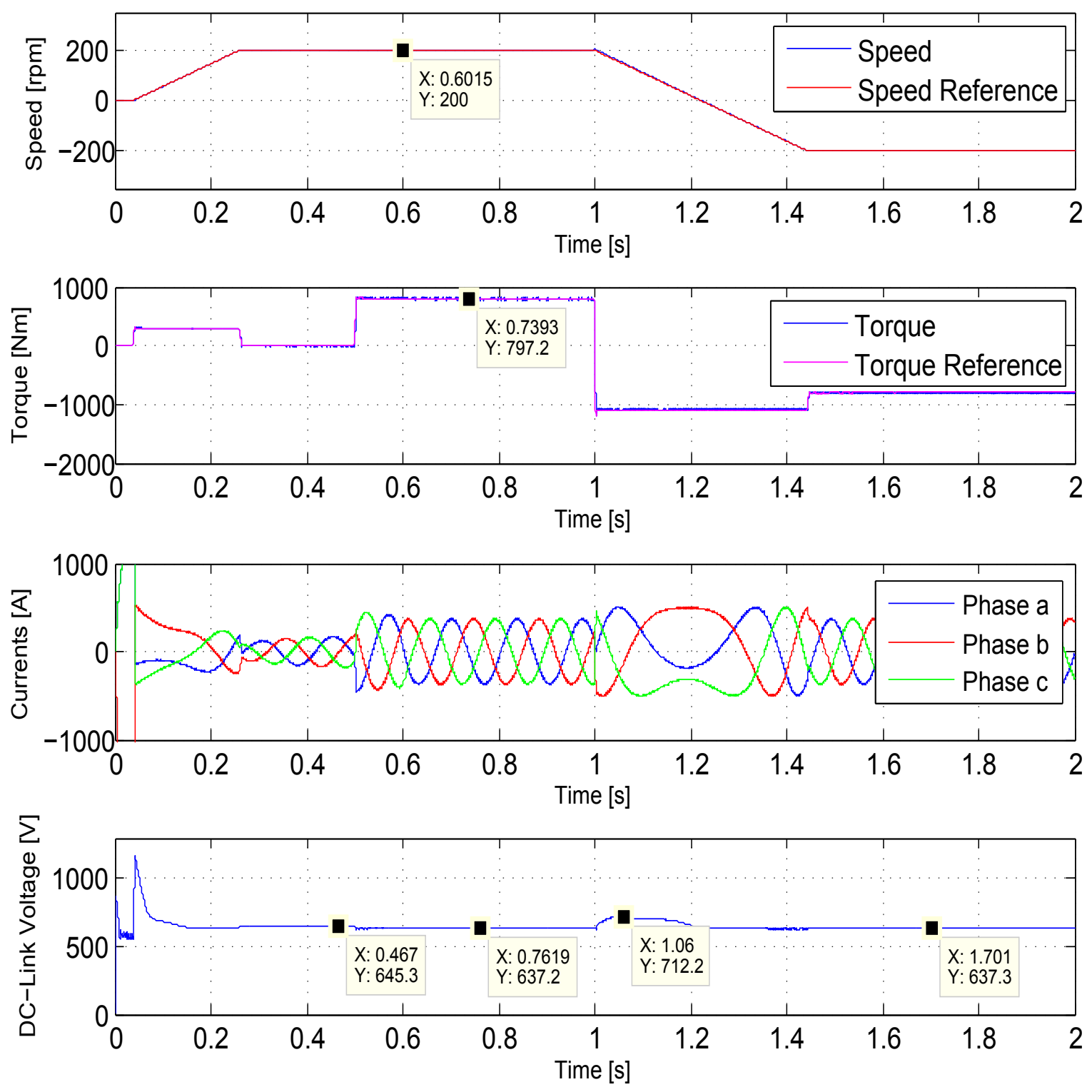

Figure 2.12: Simulation results with the FOC; speed, electromagnetic torque, currents, and DC-link voltage. 


\section{CHAPTER 3}

\section{ACTIVE COMMON-MODE VOLTAGE REDUCTION IN A FAULT-TOLERANT THREE-PHASE INVERTER}

\subsection{Introduction}

The reliability and quality of power electronic systems are of great interest to the electric drive community. Having a healthy and continuous operation of a power electronic system such as a VFD, unified power quality correction, or a hybrid electric vehicle is becoming more important. The operation of three-phase inverters in adjustable speed $\mathrm{AC}$ motor drives has a direct impact on the driven electric motors. The dynamic performance of electrical motors has been improved with the emergence of fast switching semiconductor devices such as IGBTs and MOSFETs. These semiconductor switches are controlled by the switching patterns. However, the switching patterns introduce a common-mode voltage (CMV), which causes conducted and radiated electromagnetic interference (EMI) emissions [14] and bearing breakdowns [15].

Moreover, the switching semiconductor devices in power electronics are also prone to failure. The main causes for faults in power electronics systems are semiconductor switch and capacitor failures [3]. The different faults require various remedial topology. A fault-tolerant system has remedial topologies for different type of faults, such as a switch short circuiting, a switch unable to open, a permanently open switch, 
or a failure of the gate driver circuit of the switch. In a fault-tolerant topology, any remedial solution, for either a short-circuit or an open-circuit fault adds more components to the power electronic system. In the case of both faults, the number of extra components required for the remedial topology increases substantially [16]. This chapter reviews the current state-of-the-art of fault-tolerant strategies for reliability concerns after explaining the common-mode voltage reduction methods for quality issues.

\subsection{Literature Review on Common-Mode Voltage}

In Chapter 2, the reasoning why there exists a CMV was explained. One of the main quality concerns about three-phase inverters are reducing the CMV to avoid EMI pollution and bearing breakdowns. Common-mode voltage (CMV) is defined as the instantaneous sum of the voltages from the stator winding phases to the midpoint of the DC-link, as shown in Fig. 2.11. Many studies have been completed to reduce CMV [1, 17-26]. They can be categorized as utilizing common-mode choke/transformers, cascaded inverters [17], filters [18,19], active cancellation using an extra leg [1,20], and software approaches [21-26]. Non-software methods $[1,17-20]$ reduce the CMV, but they increase the cost and complexity of the system.

Software methods, such as modulation techniques, require less complexity and change in the system [21-26]. A proper modulation technique reduces CMV to a limited voltage. Three modulation techniques were proposed by [21]. Only Near State Pulse Width Modulation (NSPWM3) is suitable for practical implementation. In this modulation technique, active zero-states are used to create a zero voltage, as shown in Figure 3.1. In the active zero-vector method, $V_{6}$ and $V_{3}$ are applied for a 
duration of $\frac{T_{0}}{2}$. This modulation uses only non-zero states in order to limit the CMV to $\pm \frac{V_{d c}}{6}$, as shown in Fig. 3.2. The duration of this active zero-vector method is $\frac{T_{0}}{2}$. The same modulation technique is called Active Zero-State Pulse Width Modulation (AZSPWM1) in [24], where a comparison among the three methods, especially in regard to the modulation index range, was reported. Some modified versions of these techniques have been reported in [22], where they focus on the mitigation of the dead-time effect.

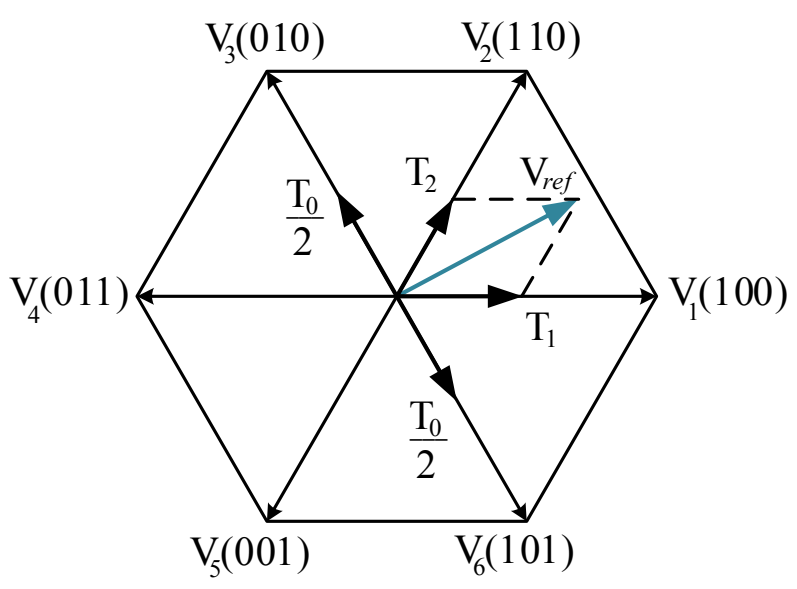

Figure 3.1: Active Zero-State pulse width modulation

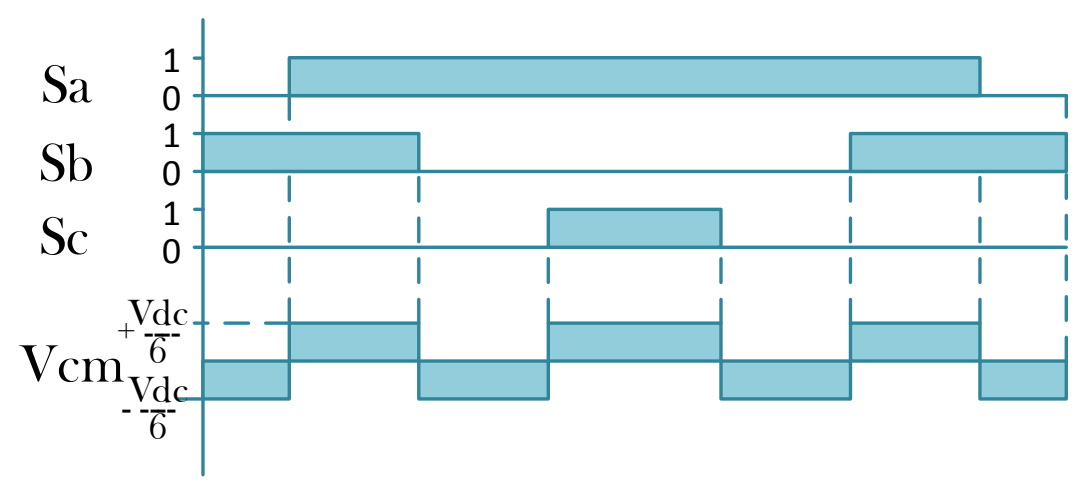

Figure 3.2: Common-mode voltage and switches status, AZSPWM modulation in sector I [1] 
The reduced CMV can be eliminated completely with filters as has been studied in [1]. In this work, a modified version of the proposed method in [1] is implemented for CMV reduction, as shown in Fig 3.3. This circuit is a four-leg three-phase inverter. This method requires the satisfaction of the following equation during each sampling period.

$$
V_{1}+V_{2}+V_{3}+V_{4}=0
$$

where $V_{i}$ and $i=1, . .4$ are the pole voltages of each leg. This equation cannot be satisfied using the conventional space vector modulation where the zero vector voltage is used. Thus, NSPWM3 (or AZSPWM) is used to satisfy Equation (3.1). The status of the gates $S_{d}$ and $\bar{S}_{d}$, as shown in Fig. 3.4, are used to satisfy this equation.

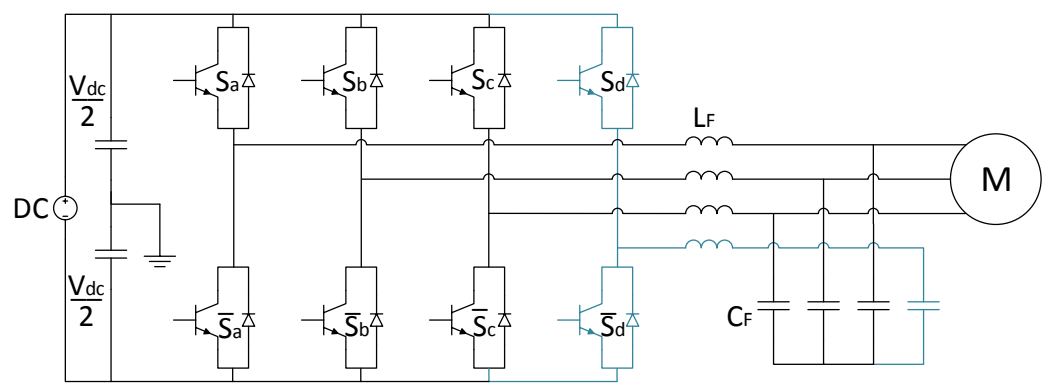

Figure 3.3: The Circuitry of the four-leg inverter showing the proposed method for CMV reduction.

They can be calculated using the following equation,

$$
S_{d}=S_{a} \oplus S_{b} \oplus S_{c}
$$

where $\oplus$ represents the xor operation and also $S_{d}$ and $\bar{S}_{d}$ are complementary. The maximum Common-Mode Voltage Reduction (CMVR) is possible if both switches are working. The top switch, $S_{d}$ applies $+\frac{V_{d c}}{2}$ to the system and $-\frac{V_{d c}}{2}$ can be applied using the bottom switch. The switch $S_{d}$ is connected to the fourth $C_{F}$. The size 


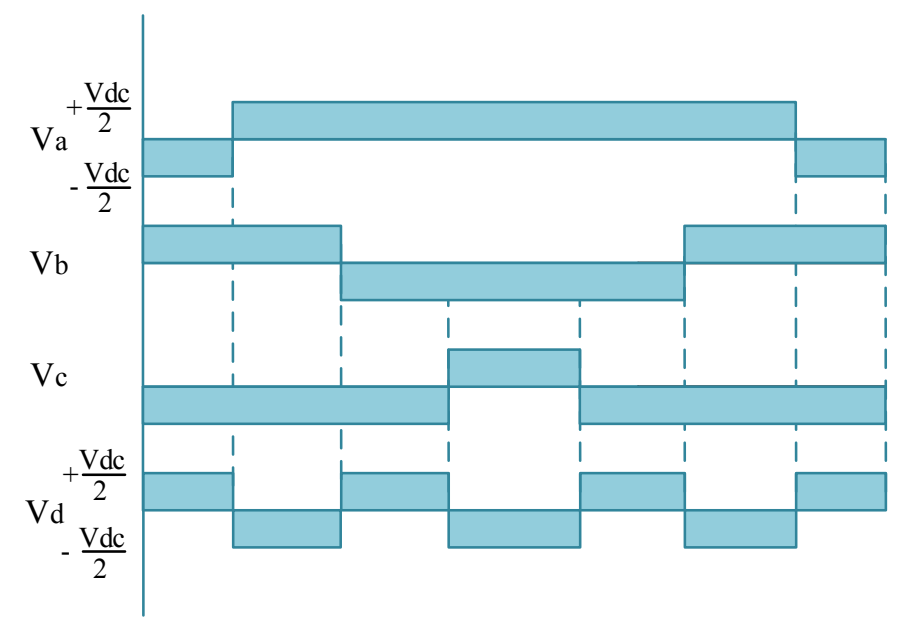

Figure 3.4: Voltage of the line to midpoint DC-link; common-mode voltage reduction proposed in [1]

of the $C_{F}$ is explained in the filter design section. This can help balance the phase voltage at the fundamental frequency.

Filters are used to ensure that the sum of each phase voltage will tend to zero and filter any high switching frequency. By using (3.2) for switch $S_{d}$, the (3.1) can be satisfied.

Harmonics of a signal such as current or voltage are frequency components in the signal that are at integer multiples of the frequency of the signal. Fourier analysis of a periodic signal presents these components and they are harmonics of the signal. Harmonic distortion shows how the signal is distorted due to the harmonics. The total harmonic distortion (THD) analysis has been carried out for both topologies. Figure 3.5 shows the magnitude and the harmonic analysis of the CMV for three-leg and four-leg inverters.

Figure 3.5a illustrates CMV pulses and the corresponding THD analysis is shown in Fig. 3.5b. Figures 3.5c and 3.5d illustrate signal and THD. The CMV of the four-leg topology does not contain any voltage pulse, so there is no $\frac{d v}{d t}$. This is due 
to the voltage applied by the leg $d$. Thus, the four-leg topology is expected to have a lower THD. The less numbers of $\frac{d v}{d t}$ yields lower THD as shown in Fig. 3.5d.

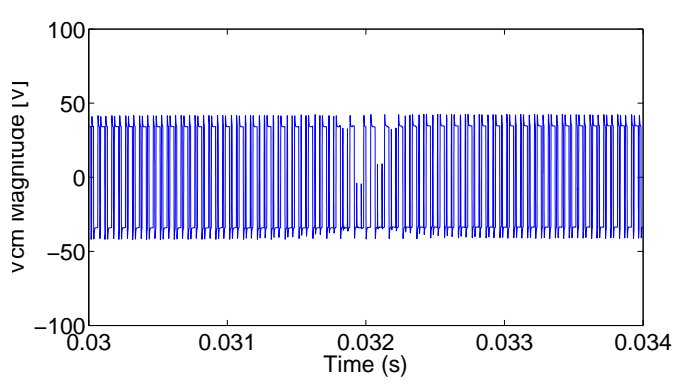

(a) Three-Leg Topology Magnitude

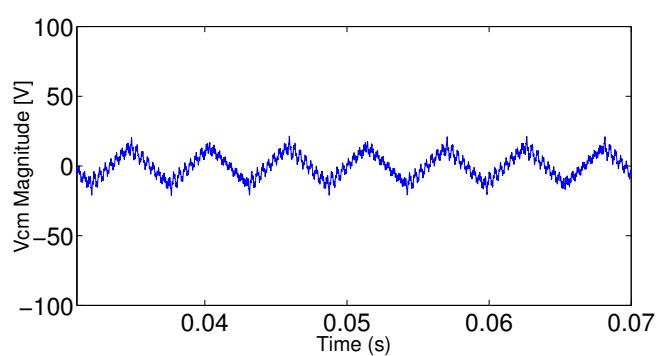

(c) Four-Leg Topology Magnitude

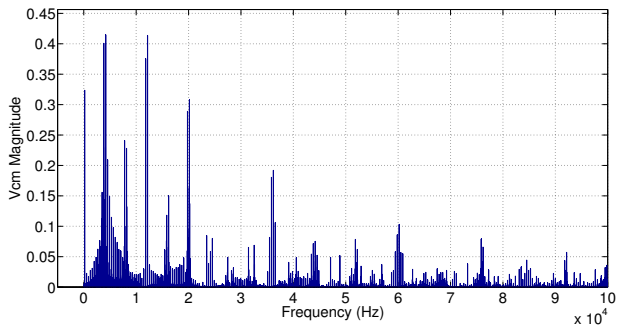

(b) Three-Leg Normalized Harmonics

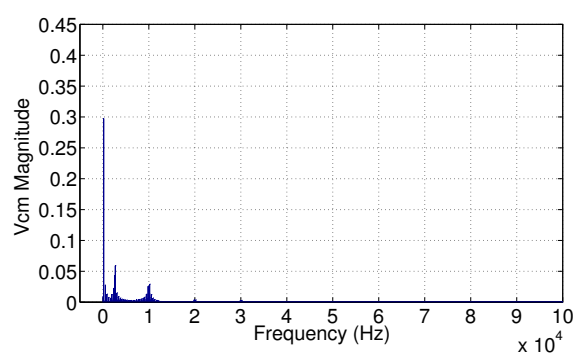

(d) Four-Leg Normalized Harmonics

Figure 3.5: Common-mode voltage using AZSPWM in different topologies

The literature review and the proposed CMVR aspect of the design has been explained in this section. Another aspect of the design is to have a fault tolerant inverter so that the fourth leg can be used when a fault is detected. In the following section, a background and a proposed design for the fault tolerant systems are given.

\subsection{Literature Review of the Fault-Tolerant Systems}

During a switch fault, the faulty switch must be isolated from the healthy system. In a three-leg three-phase inverter, once the faulty switch or leg is isolated, the drive has 
only two legs (phases) to operate. In a three-phase motor drive, the continuation of the system requires either a redundant leg or a new control algorithm where only two of the remaining healthy phases of the inverter are utilized. Each of these remedial strategies (redundant leg, two-phase operation) are reviewed in the following section.

\subsubsection{Two-Phase Operation as a Remedial Strategy}

Many remedial strategies have been proposed to improve the reliability of the electric motor drive systems after detecting a fault $[2,4,5]$. In case of a switch fault at any phase, the corresponding phase will be connected to the midpoint of the DC-link. The authors in [4] propose isolating a leg and connecting the winding of an induction machine to the midpoint of a DC-link, as shown in Figs. 3.6 and 3.7.

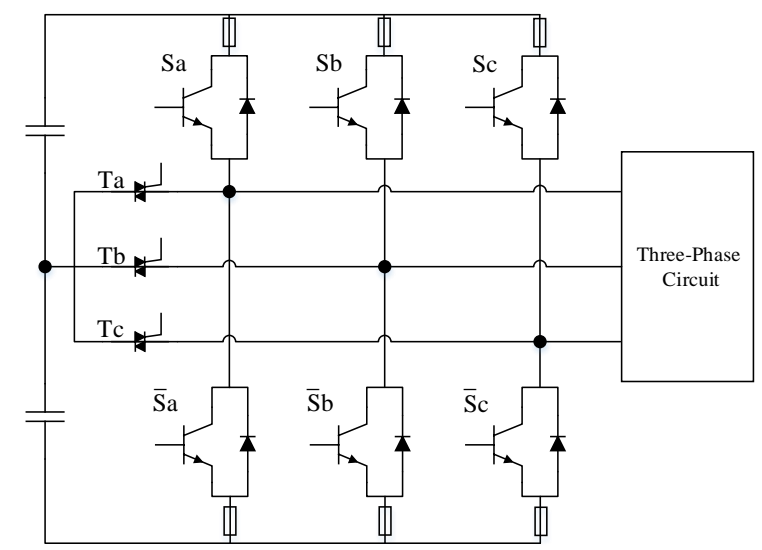

Figure 3.6: Circuit connected to DC-link mid-point

The circuit shown in Fig. 3.6 has fuses at each side of each leg. This circuit can isolate the whole leg and it is suitable for open-switch, short-circuited switch faults. This topology cannot isolate motor open-phase or short-circuited faults. 


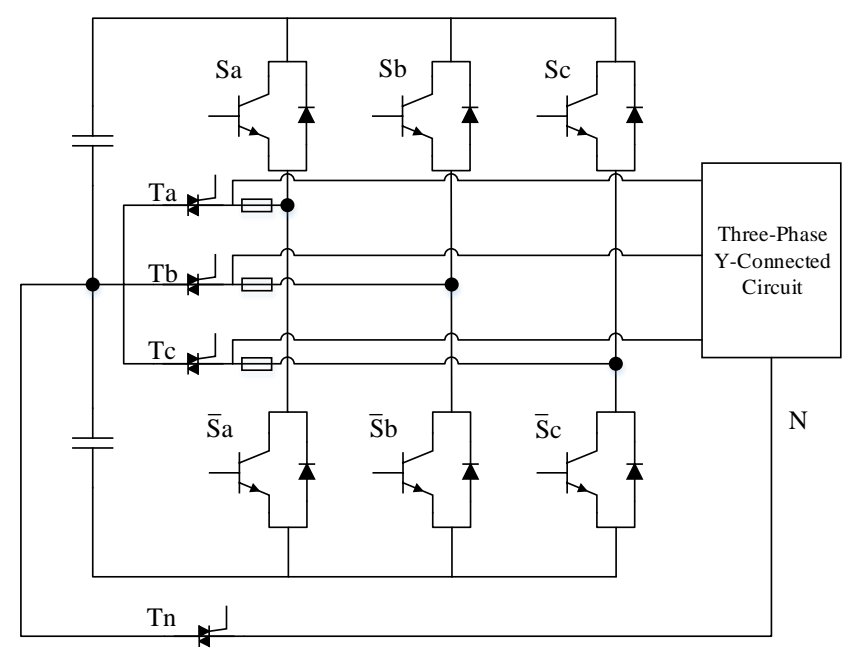

Figure 3.7: The neutral $(\mathrm{N})$ of the circuit connected to the DC-link mid-point.

By connecting the midpoint of the DC-Link to the neutral of the motor, one-phase or two-phase operation of the induction motor can be achieved. This connection eliminates the DC current caused by the fault, and the common-mode voltage, at the three-phase circuit. To achieve this, thyrister Ta must be turned on in case of a fault in leg phase $a$.

In this topology, there are no fuses for motor phase separation. Therefore, the open-phase or short-circuited phase fault cannot be mitigated. To isolate a faulty phase, the circuit shown in Fig. 3.7 is used. In this method, the neutral of the three-phase circuit must be accessible. Also, since the fuses are not located at each side of the phase leg, this circuit cannot isolate the leg when both switches are shortcircuited. However, remedial topologies for open-switch, one short-circuited switch, open-phase, and short circuited-phase are possible.

The idea of continuing the operation of a three-phase motor with only healthy two phases can be achieved by shifting the input command currents by $60^{\circ}$ from each 
other, which is $30^{\circ}$ for each phase. The stator rotating magnetomotive force (MMF) when the phases are healthy can be calculated as

$$
\mathcal{F}_{s}=N_{s} i_{a}+a N_{s} i_{b}+a^{2} N_{s} i_{c}
$$

where $a=1 \angle 120^{\circ}$ and $N_{s}$ is the number of winding turns per phase. The MMF when there is a fault in phase $a$ is:

$$
\mathcal{F}_{s}=N_{s} \cos (\theta-2 \pi / 3) i_{b}+N_{s} \cos (\theta+2 \pi / 3) i_{c}
$$

where $\theta$ is the electrical angle of the rotating MMF. To have a $60^{\circ}$ phase difference, we add $-\frac{\pi}{6}$ to phase $b$ and add $\frac{\pi}{6}$ to phase $c$. Therefore, consider input command currents of $i_{b}=I_{m} \cos (\omega t-5 \pi / 6)$ and $i_{c}=I_{m} \cos (\omega t+5 \pi / 6)$ and substituting them into (3.4), we get

$$
\mathcal{F}_{s}=\left(\frac{\sqrt{3}}{2}\right) N_{s} I_{m} \cos (\theta-\omega t)
$$

Comparing (3.5) and MMF from the healthy condition, $\mathcal{F}_{s}=(3 / 2) N_{s} I_{m} \cos (\theta \omega t)$, it is evident that for the same rating power, the currents must be increased by $\sqrt{3}$. Table 3.1 presents all of the angles that must be used for the post-fault operation.

These topologies, Fig. 3.6 and 3.7, can produce the same electromagnetic torque as before if both the DC-link voltage and switch ratings are increased by $\sqrt{3}$. Otherwise,

Table 3.1: Angles for the post-fault operation

\begin{tabular}{|c|c|c|c|}
\hline Fauled Phase & $\angle i_{a}$ & $\angle i_{b}$ & $\angle i_{c}$ \\
\hline Phase $a$ & - & $-5 \frac{\pi}{6}$ & $5 \frac{\pi}{6}$ \\
\hline Phase $b$ & $\frac{\pi}{6}$ & - & $\frac{\pi}{2}$ \\
\hline Phase $c$ & $-\frac{\pi}{6}$ & $-\frac{\pi}{2}$ & - \\
\hline
\end{tabular}


the produced electromagnetic torque has to be reduced by the factor of $\sqrt{3}$. This torque reduction is not desirable in some applications where a constant torque is required. In these topologies and their variants studied in $[2,5]$, access to the neutral of the motor stator winding or midpoint of the DC-link is required.

\subsubsection{Utilizing Redundant Leg Remedial Strategies}

Another remedial topology is to use an extra leg (phase) which can be used during the post-fault period, as shown in Figure 3.8. Employment of an extra leg in threephase inverters can provide full-load operation during the post-fault period without increasing the DC-link voltage or requiring access to the midpoint of the DC-link.

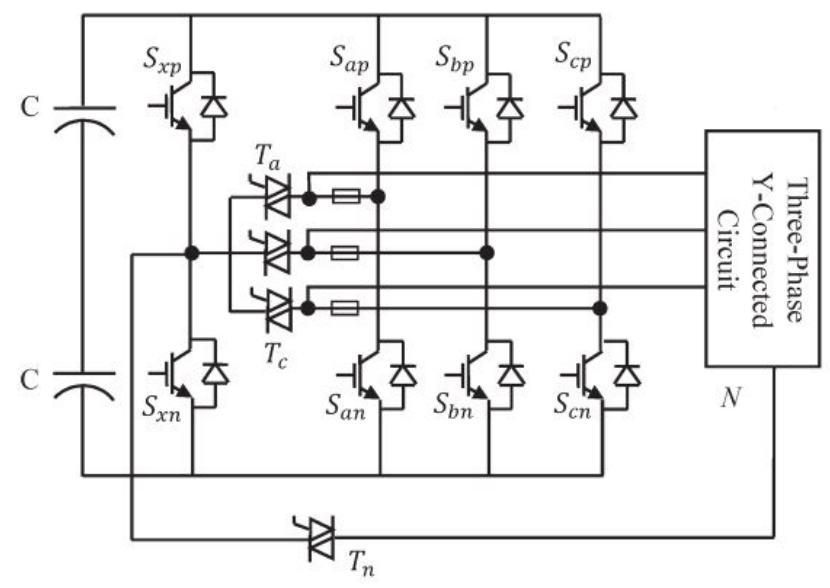

Figure 3.8: Inverter circuit with a redundant leg [2].

Most of the remedial topologies tend to isolate the whole leg of the inverter where a faulty switch is located. The circuit in 3.8 is similar to the circuit in Fig. 3.7. However, the neutral of the three-phase circuit and the phases of the inverter are connected to the extra leg instead of to the mid-point of the DC-link. This circuit is 
suitable for a fault tolerant topology where full power delivery to the load is required and the mid-point of the DC-link is not reachable.

In this section, some examples of fault tolerant topologies have been presented. These topologies do not cover all possible faults in the inverter and motor phases. The circuit proposed by [6] covers all the faults and can isolate a single switch from the circuit. The drawback of the circuit in [6] is the existence of common-mode voltage. The proposed circuitry is explained in detail in the following section. This work proposes a new circuit that not only covers all of the faults in the inverter and phase of the motor, but also reduces the common-mode voltage.

\subsection{A Proposed New Remedial Strategy}

In this paper, a fault-tolerant topology [6] is combined with a common-mode voltage reduction topology [1] in such a way that CMVR can be done even if there is a faulted switch in the four-leg inverter or a fault in the motor phases. The priority for choosing this fault-tolerant system is to have a system cover all four possible fault types, which include a short-circuited switch (short-switch), an open-circuited switch (open-switch), a short-circuited phase (short-phase), and an open-circuited phase (open-phase). The proposed topology is shown in Fig 3.9. An extra leg can also help reduce the CMV, as mentioned in the previous section. A thorough output capacity for inverter faults for different fault-tolerant topologies is studied in [5]. The only topology that is able to isolate a single switch from the circuit is the topology proposed in [6]. In this method, a faulted switch can be isolated by firing its corresponding thyristor. This scheme may be best understood by discussing the modes of operation in the following subsections. 


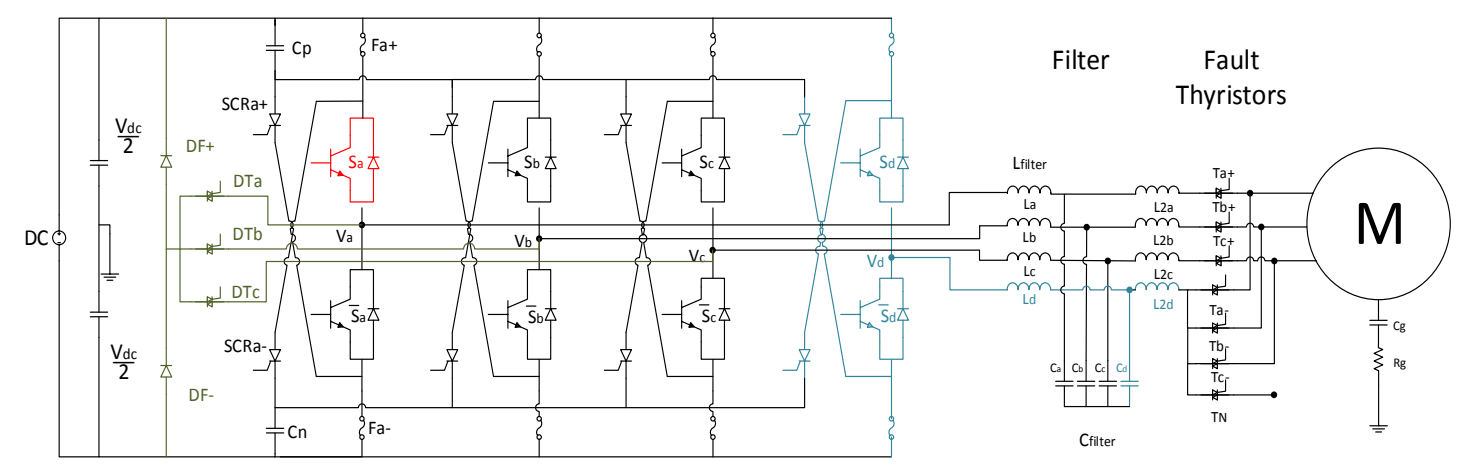

Figure 3.9: The circuitry of the proposed topology

\subsubsection{Pre-Fault Operation Mode}

Figure 3.9 shows components of this topology which consist of switches, filters, fault thyristors, and load. When the system is healthy, the fault thyristors, $T_{a}+, T_{b}+$, and $T_{c}+$ are on, and thyristors $T_{a^{-}}, T_{b^{-}}$, and $T_{c^{-}}$are off. This allows the three-phase inverter to operate normally. During normal operation, the three legs, $a, b$ and $c$, are supplying the load and the fourth leg, $d$, is reducing the common-mode current substantially, as shown in Fig. 3.5d. In this mode, CMV and the harmonic content of CMV are greatly reduced.

\subsubsection{Reconfiguration Mode}

In this mode, the circuit is reconfigured so that the inverter can continue supplying three-phase voltages to the load with minimum interruption. To avoid any damage and for safety reasons, all switches are turned off when a fault is detected. As an example, if there is a failure in switch $S_{a}$, this switch is isolated by firing the SCRa-. This will cause the fuse Fa+ to burn out. After isolation of the faulted switch in phase $a$, the fault thyristors shown in Fig. 3.9, associated with the faulted phase, 
need to be reconfigured. In this example, fault thyristor $T_{a}+$ will be turned off and $T_{a^{-}}$will be turned on. Figure 3.11 shows a post-fault circuit of short-switch of the motor phase fault after the reconfiguration mode.

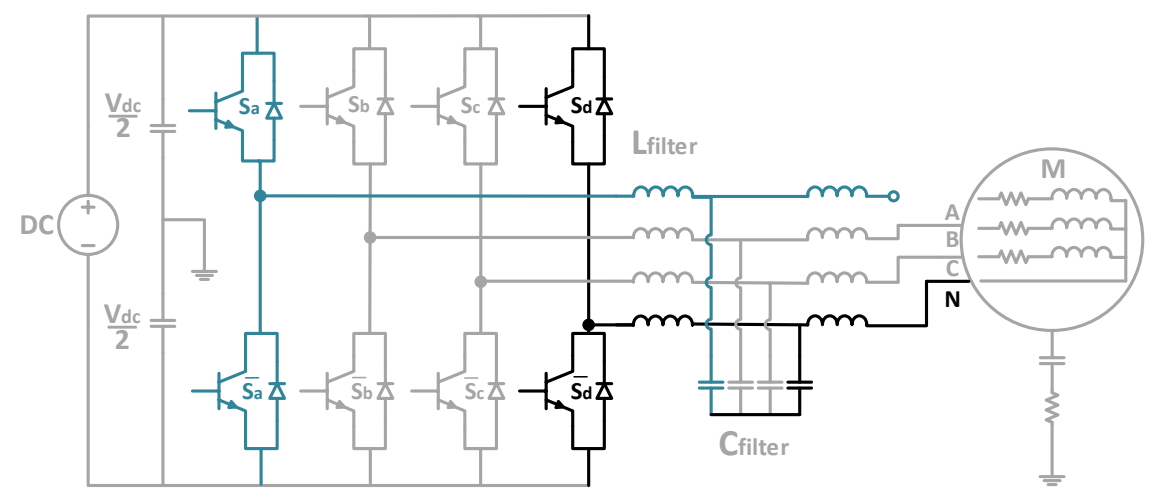

Figure 3.10: Post-Fault circuit for short-circuited or phase-open (two-phase operation)

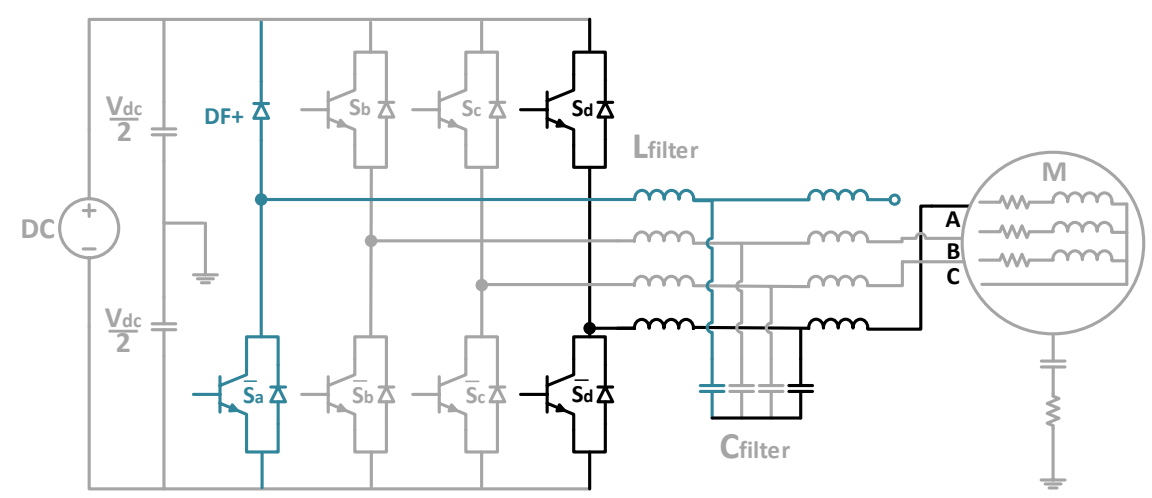

Figure 3.11: Post-Fault circuit for switch-short or open-switch

\subsubsection{Post-Fault Operation Mode}

After reconfiguring the fault thyristors, the fourth leg has been replaced with the faulty leg (in this case phase $a$ ) and the gating signals (PWM signals) of phase $a$ will now control the switches in leg $d$. This allows full operation of the inverter. Full three-phase power can be delivered to the load. In using this scheme, $C_{a}, L_{a}$, and 
$\bar{S}_{a}$ can now be utilized. These components can now be used to reduce CMV. By switching $\bar{S}_{a}$, there will be some insignificant currents in this leg (at point Va). Both positive and negative currents can flow through diodes DF + and DF- if it required. These diodes are involved in the circuitry by firing one of the thyristors $D T a, D T b$, or DTc in the reconfiguration mode. The increase in cost by adding these diodes will be insignificant.

\subsubsection{Filter Design}

Filters are used to reduce the current distortion injected to a load, or to the grid utility in the case of grid-connected systems. An LCL filter is used in this design to attenuate higher order harmonics and to reduce EMI noise. The values of $C_{a}$ and $L_{a}$ used in this design have been analyzed. Thorough analysis of LCL filter designs are studied in [27]. In the proposed design, considering the allowable current ripple of $14 \%, L_{1 a-d}$ can be found as given

$$
L_{a-d}=\frac{V_{d c}}{6 f_{s}(14 \%) I_{\max }}
$$

where $I_{\max }$ is the rated current and $f_{s}$ is the switching frequency. The resonance frequency calculated is within the constraint of $10 f<f_{\text {res }}<0.5 f_{s}$ and the resonant frequency can be calculated using the following equation

$$
2 \pi f_{r e s}=\sqrt{\frac{L_{a-d}+L_{2 a-d}}{L_{a-d} L_{2 a-d} C_{a-d}}}
$$

where $L_{2 a-d}=0.4 L_{a-d}$. The obtained values using $I_{\max }=100 \mathrm{~A}$ are given in Table 3.2. Multiple simulations have been carried out by using the values found by the 
formula in (3.7).

\subsection{Simulation Results}

A three-phase load has been simulated using three parallel resistors and inductances. Figures 3.12 - 3.13 show simulation of the DC-link voltage, CMV, common-mode current (CMC), and the current responses to short-switch, open-switch, and openphase failures. Table 3.2 provides the parameters used for these simulations. Typical detection time for an open-circuit fault is on the order of milliseconds and that of a short-circuit fault is on the order of microseconds. Since the purpose of this chapter is not fault diagnosis, the detection time of $3 \mathrm{~ms}$ for an open-circuit failure and $3 \mu \mathrm{s}$ for a short-circuit failure are chosen. In all of the simulation case studies, the faults are cleared at $t=0.04 \mathrm{~s}$ and they occur in phase $a$. In the following subsections, faults in phase and faults in switch are presented, respectively.

Table 3.2: Parameters and values used in the simulation

\begin{tabular}{c|l||c|l}
\hline \hline Parameters & Value & Parameters & Value \\
\hline$C_{d c}$ & $2 \times 1 \mathrm{mF}$ & Switching Frequency $\left(f_{s}\right)$ & $10 \mathrm{kHz}$ \\
$L_{a-d}$ & $0.25 \mathrm{mH}$ & $C_{g}$ & $3 \mathrm{nF}$ \\
$L 2_{a-d}$ & $0.1 \mathrm{mH}$ & Load Inductance & $1.04 \mathrm{mH}$ \\
$C_{a-d}$ & $15 \mu \mathrm{F}$ & Load Resistance & $0.81 \Omega$ \\
$V_{d c}$ & $210 \mathrm{~V}$ & $R_{g}$ & $900 \Omega$ \\
\hline
\end{tabular}

\subsubsection{Phase Faults}

In the case of an open-phase or short-phase fault in phase $a$ of the motor, thyristor $T_{a}+$ will be turned off so that the faulted phase is isolated, as shown in Fig 3.10. Two-phase and single-phase operations are the two possible remedial topologies when a fault occurs in one of the phases in a three-phase motor. Two-Phase operation 
requires connecting the neutral of the motor to an extra leg, which can be done by thyristor $T_{N}$. In this type of operation, CMV does not exist. However, in one-phase operation, when the neutral of the motor is not available, the CMV has one of the values of $0,+\frac{V_{d c}}{2}$, or $-\frac{V_{d c}}{2}$, which can be eliminated by a proper switching of leg $a$. The gating signals of switches in leg $a$ cannot remain the same as in the pre-fault period. During this fault, for leg $a$, changing the gating signals $S_{a}$ and $\bar{S}_{a}$ to $\bar{S}_{b}$ and $S_{b}$ and for leg $d$ changing the gating signal from $S_{d}$ and $\bar{S}_{d}$ to $\bar{S}_{c}$ and $S_{c}$ yields to elimination of the CMV. The CMV during post-fault remains with zero pulse, as shown in Fig 3.12. Once the fault has been cleared, the new two-phase operation command presented in Table 3.1 is used for the motor control. During post-fault two-phase operation, the currents are shifted by $180^{\circ}$, as shown in Fig 3.12 .
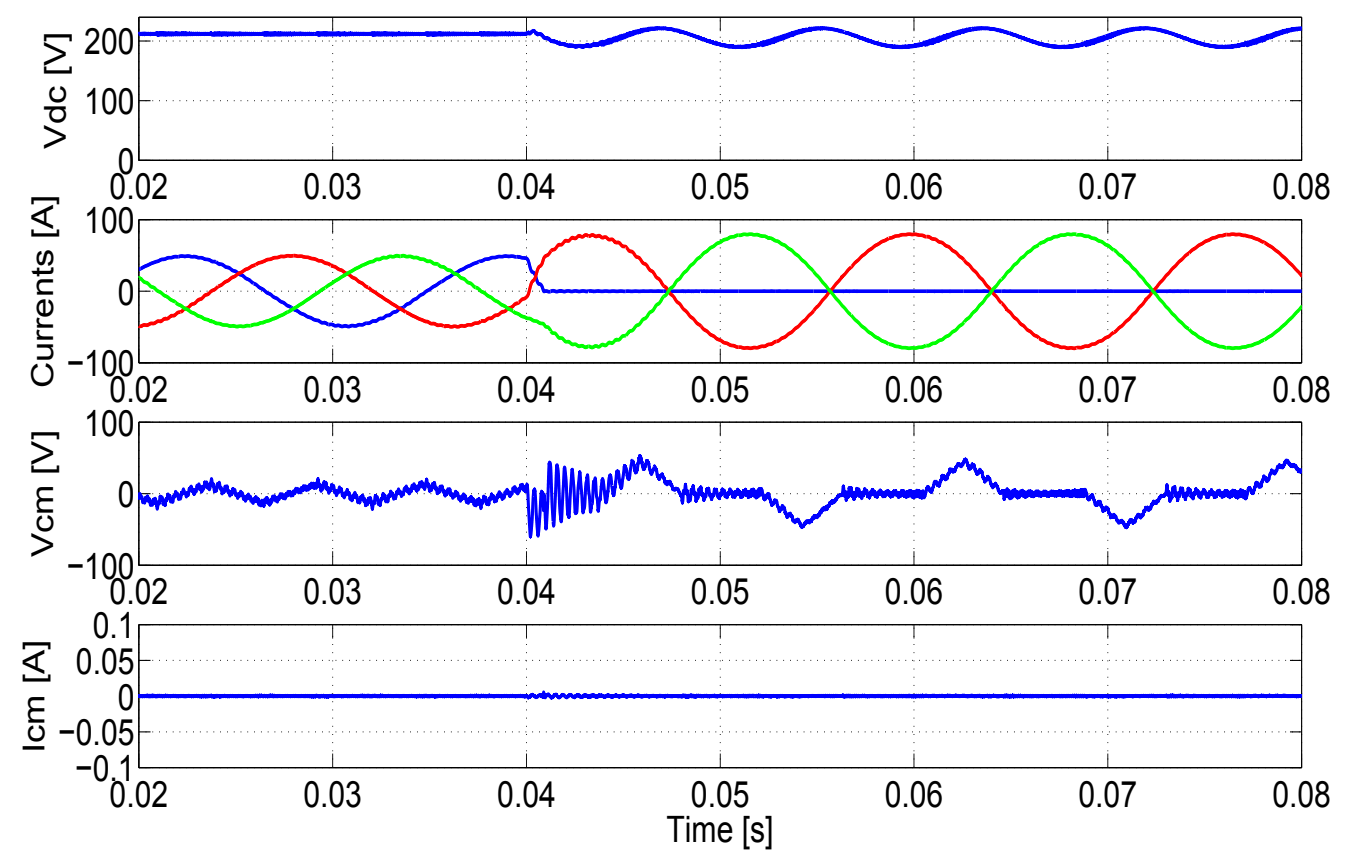

Figure 3.12: Open-circuit phase $a$ failure

The procedure for the open-phase fault remediation of the proposed circuit has 
been presented in this subsection. In the following subsection, the switch fault postfault operation is explained.

\subsubsection{Switch Faults}

Once the fault has been diagnosed, the faulty switch is isolated from the faulted leg and the healthy switch in the faulted leg can still be used to reduce the common-mode voltage, as shown in Fig. 3.11 and 3.14. Two scenarios of the open-switch and short-circuited fault have been simulated. In both cases, the faulty switch gets isolated and the new circuit is configured so that the motor drive can deliver full power to the load. As shown in Fig. 3.13 and 3.14, the CMV and CMC in this type of fault cannot be zero. However, the the CMV and CMC are reduced by utilizing the remaining healthy switch of the phase $a, \bar{S}_{a}$.

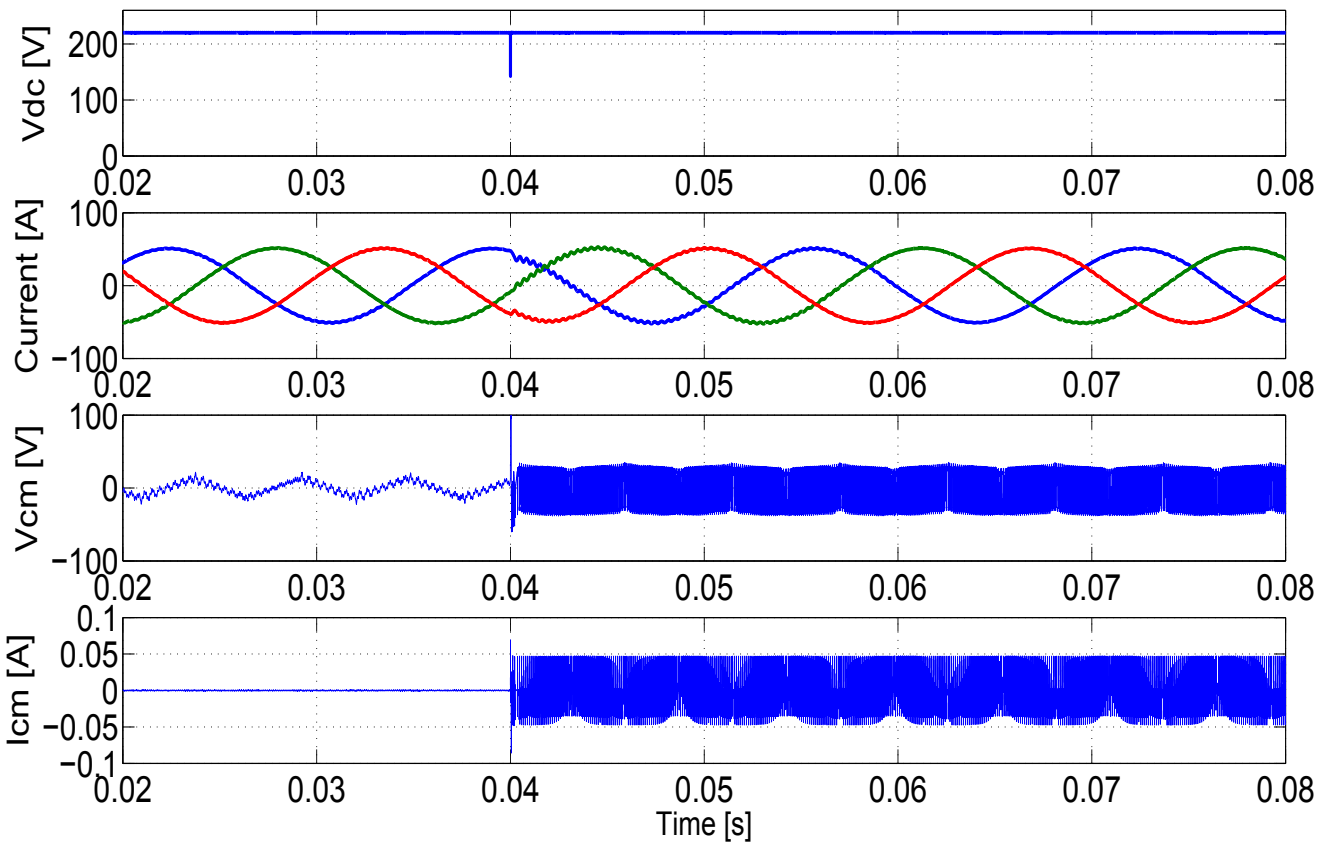

Figure 3.13: Short-circuit switch failure 


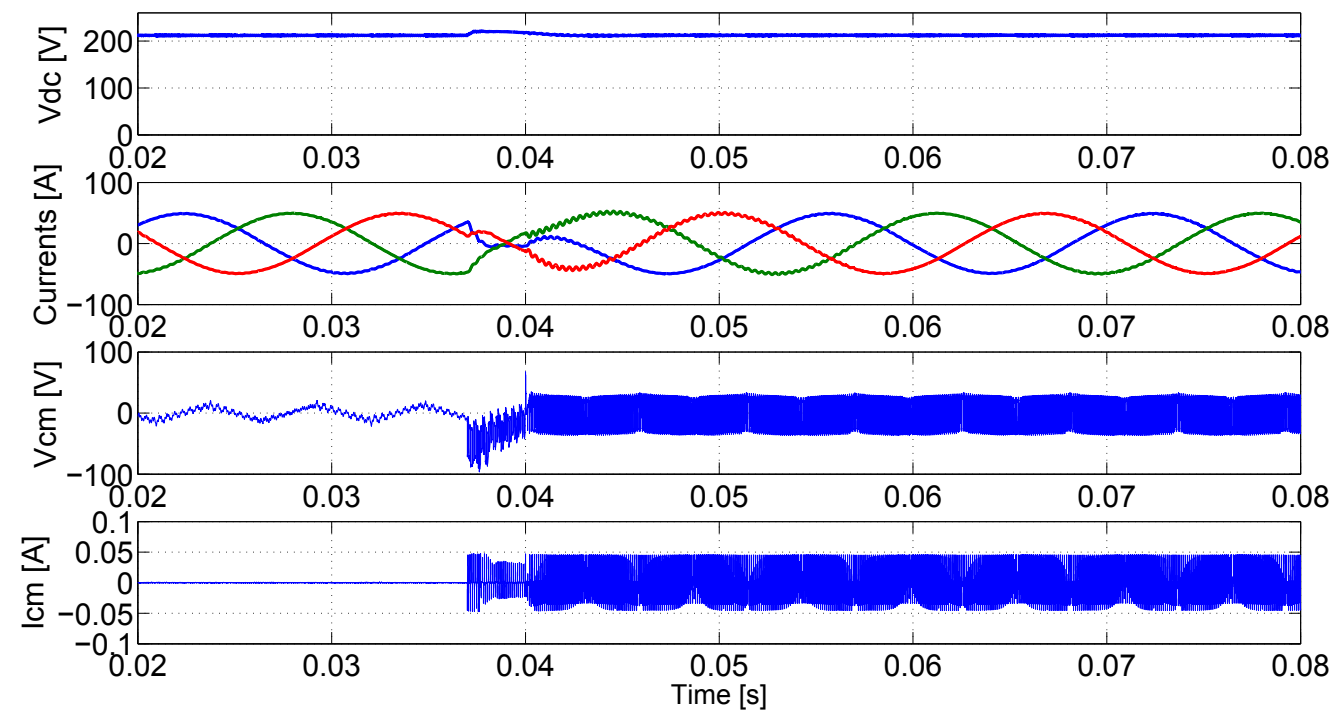

Figure 3.14: Open-circuit switch failure

The magnitude and number of spikes in the $\mathrm{CMC}$ has been reduced due to the reduced magnitude and the number of $d v / d t$ in the CMV. The CMV and the CMC are reduced by about $25 \%$ in magnitude and by around $50 \%$ reduction in the number of $d v / d t$ caused by CMV compared to the three-leg topology.

The effect of using the healthy switch, in this example $\bar{S}_{a}$, on CMV and CMC can be seen in Fig. 3.15a and 3.15b. The gating signal of switch $\bar{S}_{a}$ now will be the same as $\bar{S}_{d}$ during pre-fault. In Fig 3.16a, the magnitude of the current in each leg is shown. Figure $3.16 \mathrm{~b}$ shows the THD of the load current at different modulation indexes.

This remedial technique is capable of mitigating all of the aforementioned faults. However, the design of this inverter becomes more complex compared to that of a conventional two-level three-phase inverter. The efficiency of this design must also be calculated and compared to the other remedial techniques considering the triacs are in series. 

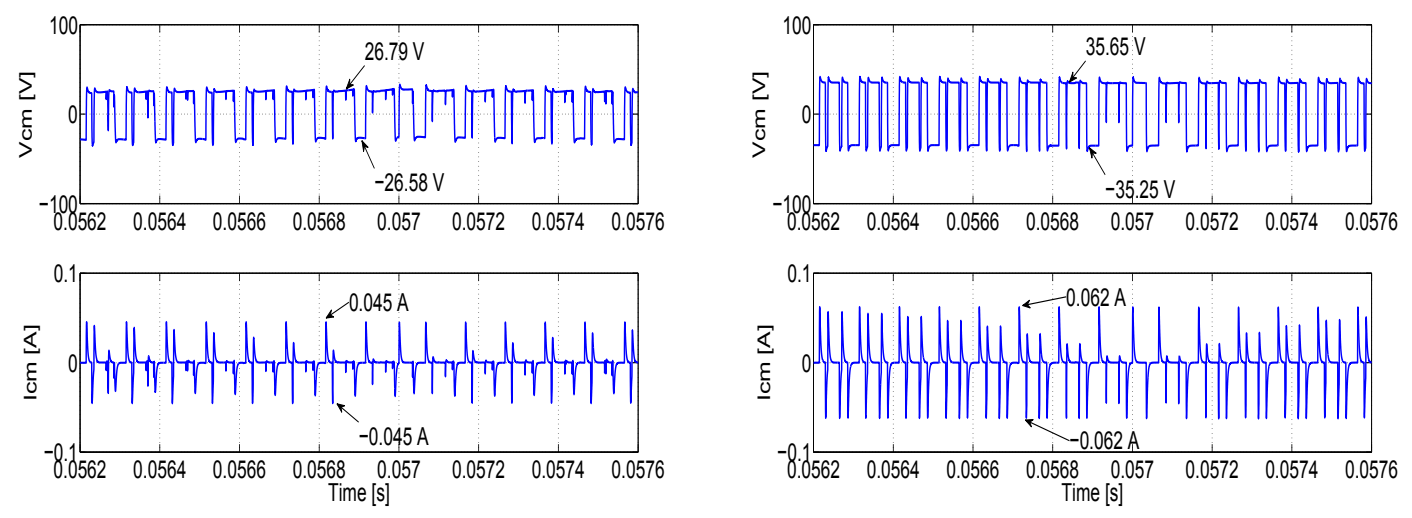

(a) CMV and CMC with utilizing $\bar{S}_{a}$

(b) CMV and CMC without utilizing $\bar{S}_{a}$ (threeleg inverter)

Figure 3.15: Comparison of CMV and CMC

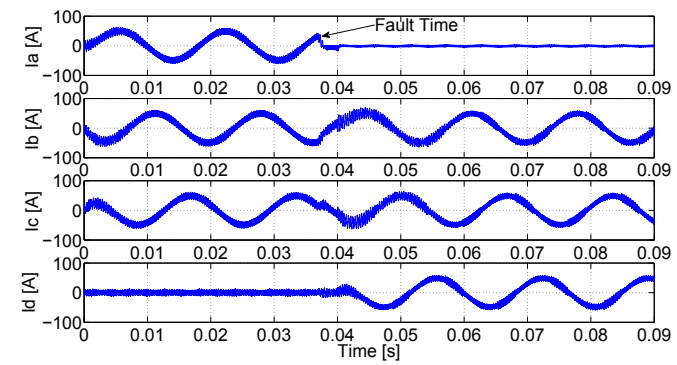

(a) The current in each leg during pre- and postfault

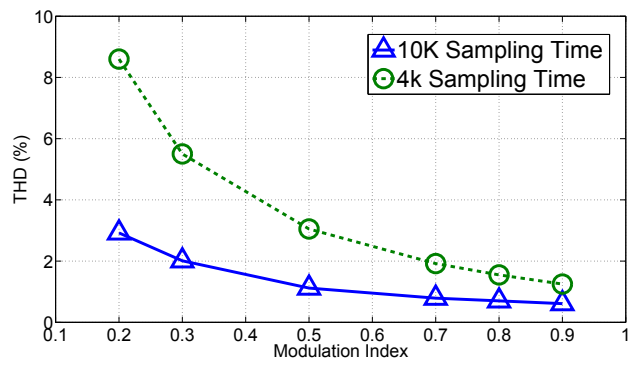

(b) THD for multiple modulation index

Figure 3.16: Four-leg inverter currents and THD

\subsection{Conclusion}

The proposed fault-tolerant topology is able to clear open-switch, short-switch, shortphase, and open-phase faults. The common-mode voltage and current are minimum during pre-fault. Using this topology and the new switching strategy, common-mode voltage and current are eliminated during post-fault operation of the inverter for openphase and short-phase faults and reduced for open-switch and short-switch faults. 


\section{CHAPTER 4}

\section{NEW OPEN-SWITCH FAULT DETECTION METHOD}

\subsection{Introduction}

Variable-frequency drives (VFDs) are widely used for the control of electrical machines such as induction motors (IMs) or permanent-magnet synchronous motors (PMSMs). These VFDs are essential components in many industries, where they are used to control the speed and electromagnetic torque of electrical machines such as in electric vehicles. Similar to other electrical devices, these drives are subject to failure due to several types of faults, such as a switch fault or a DC-link fault [3]. These faults can yield catastrophic consequences if proper remedial action is not immediately taken. Moreover, any unexpected interruption of a VFD could lead to an expensive halt of a production line. Figure 4.1 shows a simplified VFD system with a two-level voltage source inverter, a DC-link capacitor, switches, an electrical motor, and a mechanical load attached. Faults can occur in any one component of the whole system.

In the two-level voltage source inverter, there are six controlled switches which apply the desired three-phase voltage waveforms to the motor. A fault at any switch, for instance switch $S_{1}$, could cause the switch either to remain conducting as a shortcircuited switch or to remain open, where no current is conducted through the switch and it acts as an open switch. Localizing the faulty switch will help the system take 


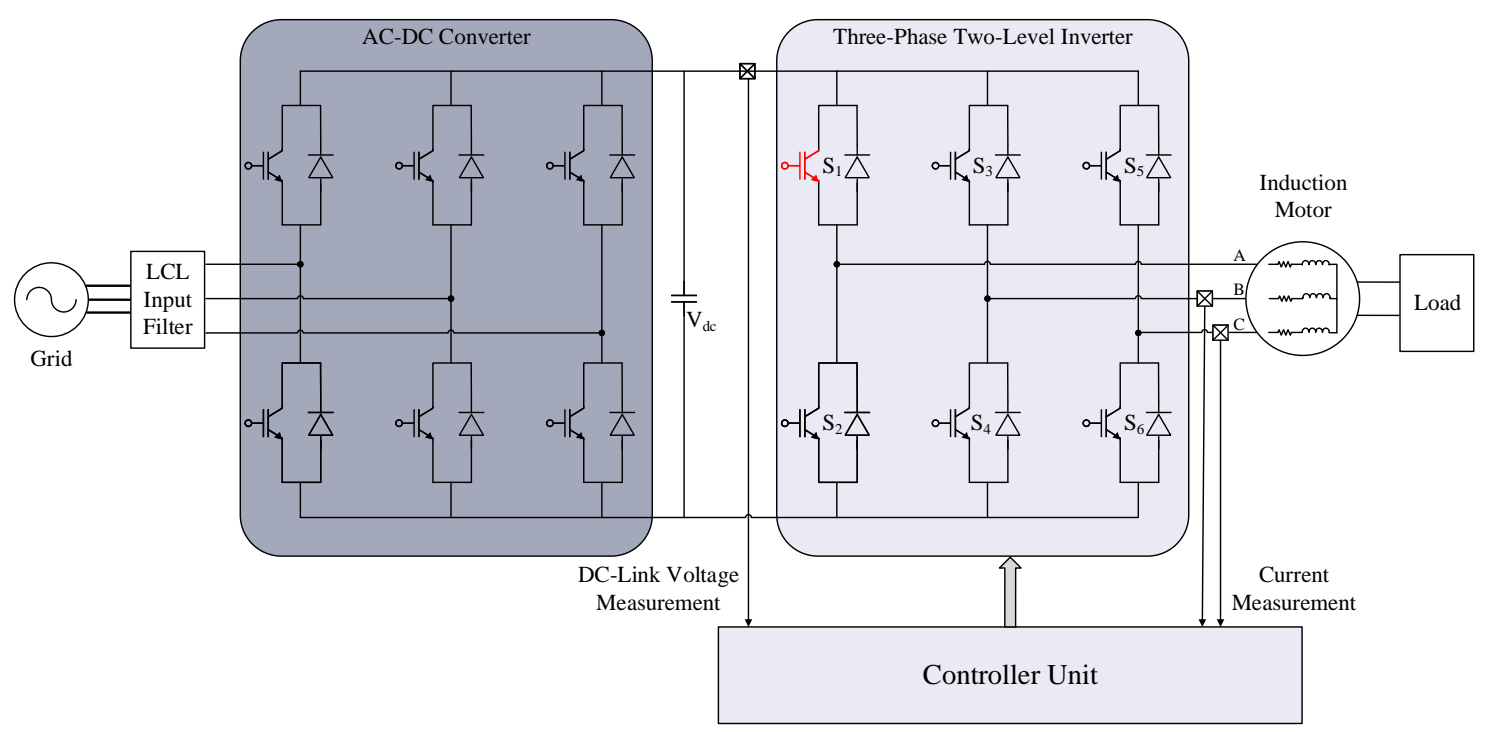

Figure 4.1: Simplified three-phase two-level inverter with fault at switch $S_{a}$

an appropriate remedial action in order to remain operating and safe.

Fault-tolerant systems exist that provide temporary solutions for avoiding an unexpected stop of the production line. Fault-tolerant drive systems are designed to minimize such incidents and also to deliver power to consumer loads in a reliable manner. These fault-tolerant systems use diagnostic techniques to detect the occurrence of a faulty state. New detection methods are often being investigated and implemented [28-36]. These detection methods are based on measuring currents [28-31], sensing the voltage output [32], utilizing the applied reference voltage vector [33,34] and using artificial neural networks $[35,36]$. These methods identify and localize one or multiple faulted switches in a three-phase voltage-source inverter. Various techniques of IGBT open-switch fault detection have been summarized in [37]. Fault detection is usually done in three domains: time domain, frequency domain, and time and frequency domain, where the measured currents and voltages are processed in either the time or frequency domains to extract the fault signature. In this work, the time domain 
is used to extract the faulty signatures.

The open-switch circuit faults in a three-phase inverter lead to a DC current offset in both the healthy and the faulty phases of the inverter which is connected to the motor windings. Figure 4.2 depicts three-phase currents of an induction motor during the pre- and post-fault condition for an open-switch $S_{1}$. During the post-fault period $(t>2.907 s)$, the positive current cannot flow through the $S_{1}$ switch because $S_{1}$ is faulty. The switches $S_{1}$ and $S_{2}$ are located in phase $a$. By taking the average of phase $a$, it can be seen that a DC current will be introduced in the load. The interaction between this DC component and the rotating field generates a pulsating torque at the stator current frequency. Therefore, the DC current reduces the maximum average torque available to the drive. An unequal stress on the remaining healthy components of the drive may be applied due to this DC current, which may lead to a secondary fault.

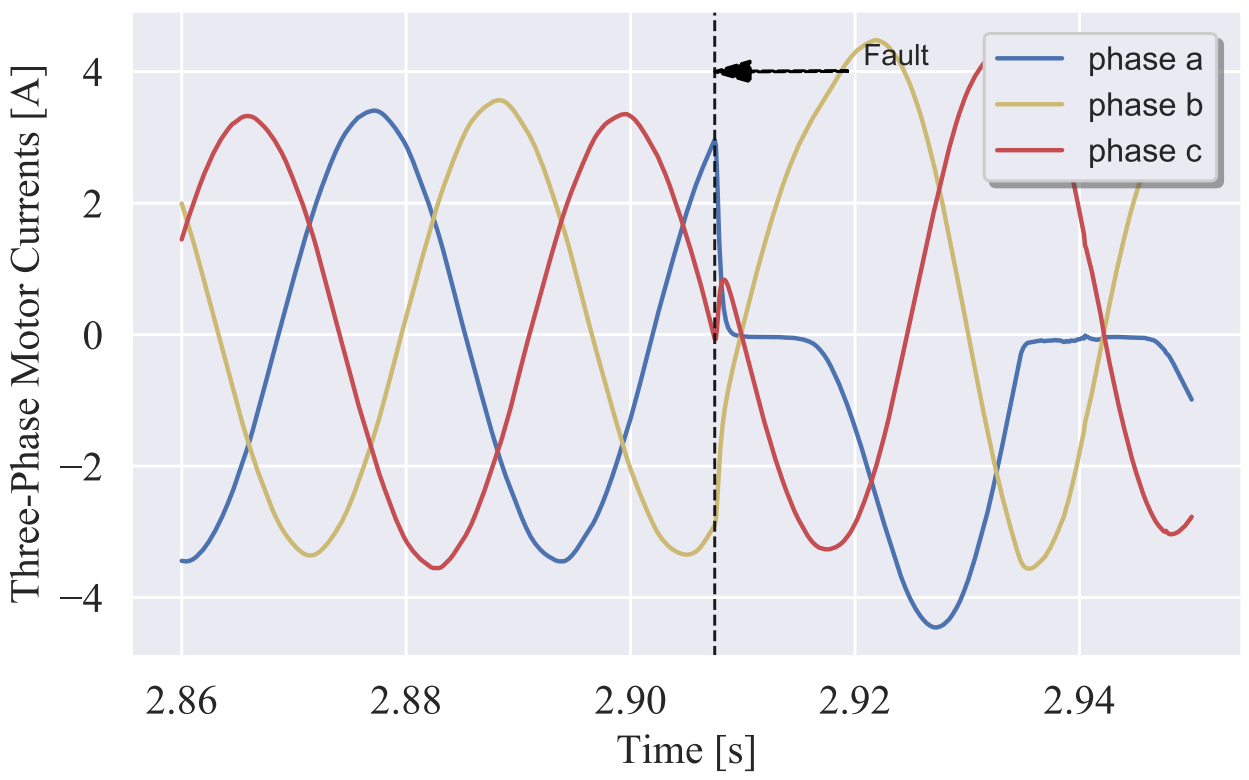

Figure 4.2: Simulation of three-phase current in pre- and post-fault period 
This work presents a new fault diagnosis algorithm to detect an open-switch that is based on three-phase currents measurements of a voltage source inverter. The measured data is then processed to extract fault signatures. The obtained three-phase currents and their covariance values are continuously monitored so that any deviation from a healthy signature will be an indication of a fault occurrence. In a balanced three-phase system, the currents induced in the motor are symmetrical and shifted by $120^{\circ}$ from each other. By computing the absolute value of the covariance of the normalized three-phase currents, a new signal is obtained that oscillates six times faster than any of the phase currents. Using this fast oscillating signal along with current values as the signatures in a look-up table, a fast detection algorithm is possible.

This chapter is organized as follows: The related work and literature review is presented in section 4.2. The methodology of the proposed technique is discussed in section 4.3. The experimental results and discussions are presented in section 4.4 .

\subsection{Literature Review of the Open-Switch Fault}

The author of [3] reports a survey where $38 \%$ of the faults in a VFD are caused by power switch failures. Faults on IGBT switches or the DC-Link capacitor in electrical drives could cause explosions inside the drives and their surroundings if they are not detected soon enough.

Two types of invasive and non-invasive approaches are used for fault detection in the literature. In any fault detection method, it is desirable to detect the anomaly as fast as possible. Should the detection algorithm require data for one period of the motor current, the detection time will vary for different operating speeds. For 
a 4-pole, $60-\mathrm{Hz}$ machine, the nominal speed is about $1800 \mathrm{rpm}$. and in a VFD, the frequency of the motor current can vary from $1 \mathrm{~Hz}$ to $60 \mathrm{~Hz}$. Therefore, if the speed of the motor is around $1 \mathrm{~Hz}$, the detection algorithm may take a few seconds to detect a fault.

Several approaches for the detection of an open-switch fault have been developed [28-37]. These approaches are reviewed in this section, particularly, from their methodology aspects and their time required for a detection. Most of these works utilize the Concordia transformation to convert the three-phase coordinate system $(a b c)$ to an $(\alpha \beta)$ coordinate system. The currents in the $\alpha \beta$-coordinate system for each faulty switch are shown in Figure 4.3. During the pre-fault period, each current trajectory describes a circle and during the post-fault period, describes a half-circle with a straight line. This straight-line angle changes depending upon which one of the six switches is faulty.

Fault localization in Mendez [28] is done by averaging each phase of the current over one period. A Park vector transformation is used to calculate the magnitude and phase angle of the current. Based on these calculated values, a look-up table identifies the faulty switch. Since the averaged current is a load-dependent parameter, by normalizing this current, a slight improvement in reliability can be obtained. However, it still performs poorly for small currents. The detection time of this method and the normalized DC method have been reported to take about $20 \mathrm{~ms}$ and $18 \mathrm{~ms}$ [29], respectively.

Peuget et al. [30] proposed two knowledge-based techniques based on the analysis of the current-vector trajectory (slope, $\Psi$ ) and the instantaneous frequency in a faulty mode of operations. To find the current-vector trajectory, utilizing $i_{\alpha}$ and $i_{\beta}$, the following formula is used: 

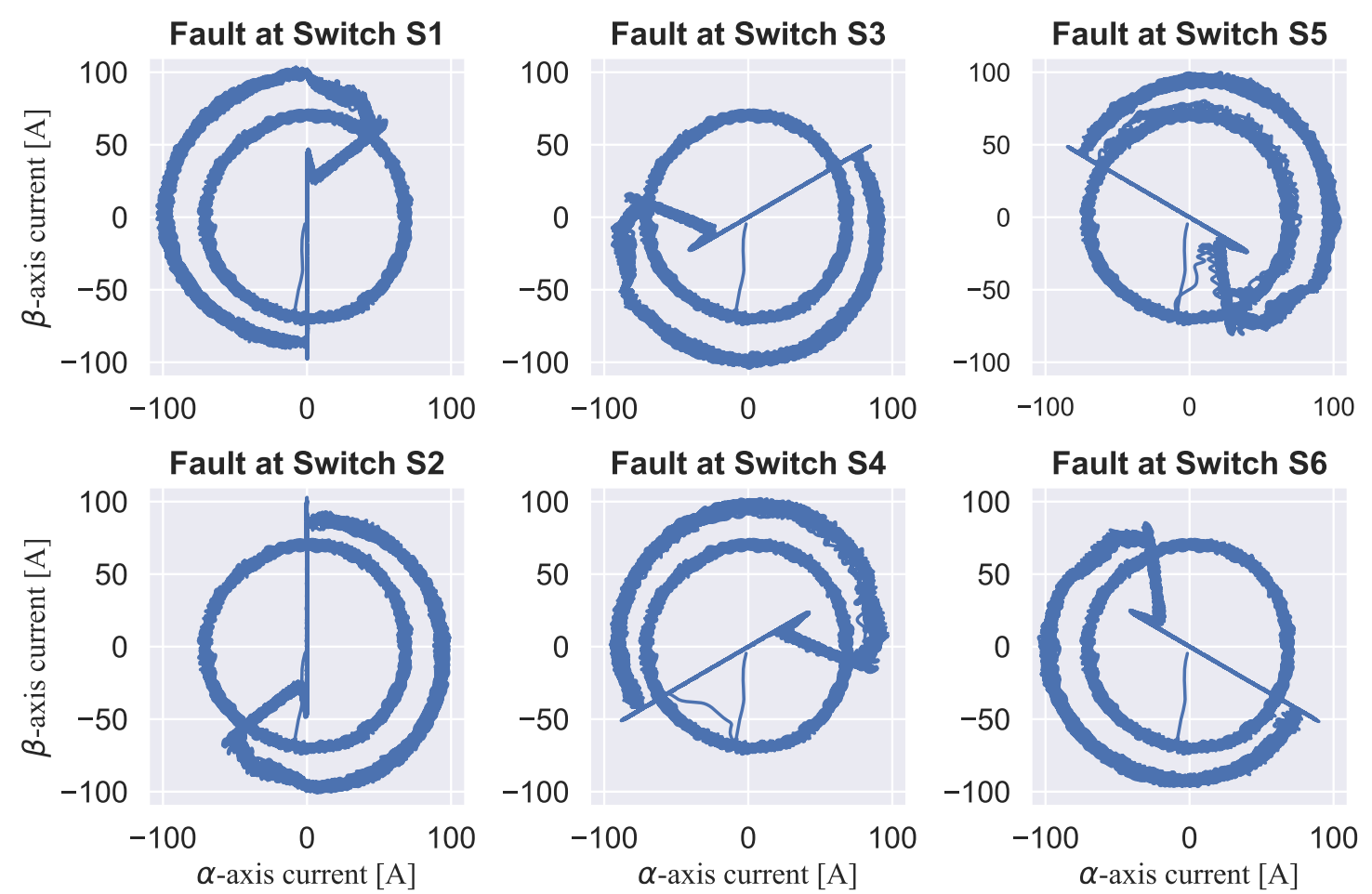

Figure 4.3: Healthy and faulty current in $\alpha \beta$-plane for each fault; $\omega_{r}=900 \mathrm{rpm}$ and $T e=79 \mathrm{Nm}$

$$
\Psi=\frac{i_{\alpha_{k}}-i_{\alpha_{k-1}}}{i_{\beta_{k}}-i_{\beta_{k-1}}}
$$

where $k$ and $k-1$ are the current and previous time samples. The observation of the current trajectories shows that $\Psi$ is constant during half of the current period, as shown in Fig. 4.3. Its specific value is used to determine the faulty switch. This technique requires one full current period of the fundamental frequency for detection.

Trabelsi et al. [31] have expanded the slope technique proposed by [30] to detect two simultaneous open-switch faults. This modified slope method uses a Schmitt trigger to localize the faulty transistor. In reference [32], a fast detection time has been obtained by an approach where three voltage sensors in the lower switches 
are installed. This method is considered invasive due to the additional circuitry required. The authors in [33] propose an approach based on utilizing a reference voltage vector. A Field-Oriented Control (FOC) simulation for an IM has been carried out to demonstrate its effectiveness. The authors in [34] have utilized the same approach as in [33] and its effectiveness is verified by implementing this method. Their simulations and experiment results for low speeds and transient conditions have not been documented. This method detects the fault in about one period of the fundamental stator current for all operating speeds.

The authors in [35] and [36] use Artificial Neural Networks (ANNs) for fault classification with current and voltage signals as their detection signals. A model-based drive system [35] is used to simulate the voltage and current of each phase. This data is used for fault identification and ANN training. The authors also use a field-oriented closed-loop control algorithm to obtain the current and voltage needed for training. In reference [36] a three-phase RL circuit is used to simulate the pre- and post-fault currents and voltages needed to train an ANN. Both of these methods are invasive approaches since they require additional voltage sensors in real-time applications.

Model-based techniques are outstanding if an accurate model of the process can be obtained. In an induction motor drive, an accurate model of the whole system is difficult to obtain. If the detection method is dependent on the machine parameters, a change in any of the parameters of the machine, such as an increase in temperature or utilizing a different machine, lead to a false identification.

Table 4.1 summarizes the algorithms and compares their detection time and methodology. It can be noted that their detection times are in the range of $16 \mathrm{~ms}$ to $20 \mathrm{~ms}$, except for the invasive approach in [32]. 
Table 4.1: Fault detection timing for different methods in literature

\begin{tabular}{ccc}
\hline Methods & Detection Time & Detection Parameter \\
\hline \hline Park Vector Method [28] & $>20 \mathrm{~ms}$ & Three-Phase Current \\
\hline $\begin{array}{c}\text { Normalized DC } \\
\text { Current Method [29] }\end{array}$ & $18.4 \mathrm{~ms}$ & Three-Phase Current \\
\hline Slope Method [30] & $38.3 \mathrm{~ms}$ & Three-Phase Current \\
\hline $\begin{array}{c}\text { Modified Slope } \\
\text { Method [31] }\end{array}$ & $20 \mathrm{~ms}$ & Three-Phase Current \\
\hline $\begin{array}{c}\text { Sensing Voltage Across } \\
\text { the Lower Switch [32] }\end{array}$ & $2.7 \mathrm{~ms}$ & $\begin{array}{c}\text { Lower Switch } \\
\text { Voltage sensors }\end{array}$ \\
\hline $\begin{array}{c}\text { Voltage vector location } \\
\text { Based Model [33,34] }\end{array}$ & $16 \mathrm{~ms}$ & $\begin{array}{c}\text { Three-Phase Voltage } \\
\text { Reference Location }\end{array}$ \\
\hline Model Based ANN Method [35] & $16 \mathrm{~ms}$ & $\begin{array}{c}\text { Three-Phase Voltage } \\
\text { and Current }\end{array}$ \\
\hline Model Based ANN Method [36] & $20 \mathrm{~ms}$ & $\begin{array}{c}\text { Three-Phase Voltage } \\
\text { and Current }\end{array}$ \\
\hline
\end{tabular}

\subsection{Proposed Method}

This work proposes a non-invasive standalone solution for localizing a faulty switch in a two-level inverter using only the three-phase currents. This solution is independent of motor characteristics such as motor parameters. The detection time varies based on when the response of the current gets distorted and these currents are different from the healthy three-phase currents. For instance, when a fault happens on one of the top switches $(S 1, S 2$, and $S 3)$ and if their corresponding phase currents are negative, the effect of the fault is negligible. Therefore there will be a delay in detecting the fault. An important criterion in comparing the detection time is when a fault occurs. For instance, in switch $S 1$, a fault can occur when $i_{a}$ is positive or negative, which has a profound impact on the detection time. The term fault-angle will be used to describe the status of the phase current when a fault occurs. Figure 4.4 illustrates current of the phase $a, i_{a}$, with its fault-angle. To evaluate the algorithm performance 


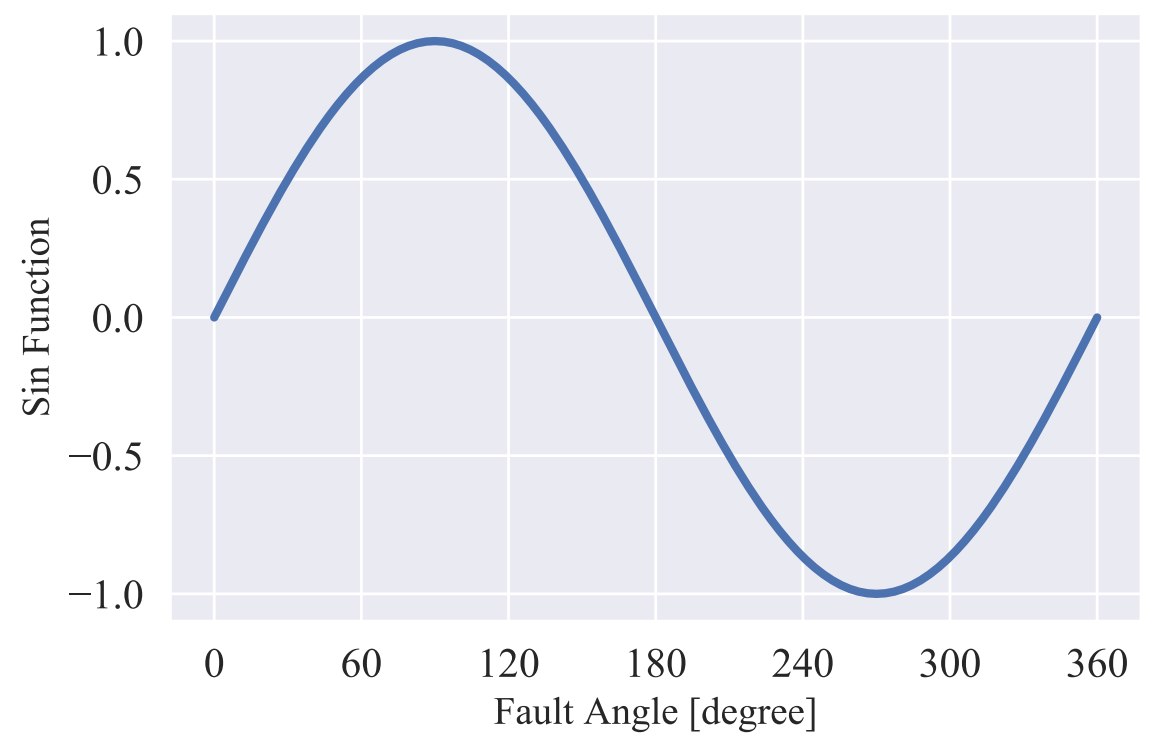

Figure 4.4: Fault angle illustration with respect to the phase $a$

in terms of detection time, multiple faults will be introduced at different fault angles.

In a healthy system, the instantaneous values of the currents in a three-phase induction motor can be found using

$$
\begin{aligned}
& i_{a}(t)=\Re\left\{I_{m} \angle(\omega t)\right\} \\
& i_{b}(t)=\Re\left\{I_{m} \angle\left(\omega t-\frac{2 \pi}{3}\right)\right\} \\
& i_{c}(t)=\Re\left\{I_{m} \angle\left(\omega t+\frac{2 \pi}{3}\right)\right\}
\end{aligned}
$$

where $\omega$ is the synchronous angular velocity of the motor currents. The data sets obtained from the three-phase currents are utilized to create a new detection feature. This feature is obtained by multiplying two of the phases together. The matrix, $\mathbf{M} \in \mathbb{R}^{\mathbf{2} \times \mathbf{3}}$, has two columns, where the first column of this matrix consists of all of the instantaneous values of the three-phase current, $i_{a}(t), i_{b}(t)$, and $i_{c}(t)$. The second 
column consists of multiplication of two of the phases together, $i_{a}(t) i_{b}(t), i_{b}(t) i_{c}(t)$, and $i_{c}(t) i_{a}(t)$. Since these currents are multiplied by each other, the second column constructs data points with a frequency higher than the frequency of the original currents. By using this higher frequency feature, a faster detection is possible. The instantaneous values of $i_{a}(t), i_{b}(t)$, and $i_{c}(t)$ and their multiplications are used to construct the matrix shown in Equation (4.18). The matrix $M$ is represented as follows

$$
\mathbf{M}=\left[\begin{array}{cc}
i_{a}(t) & i_{a}(t) i_{b}(t) \\
i_{b}(t) & i_{b}(t) i_{c}(t) \\
i_{c}(t) & i_{c}(t) i_{a}(t)
\end{array}\right]
$$

and, to make the notation easier, is written

$$
\mathbf{M}=\left[\begin{array}{ll}
X & Y
\end{array}\right]
$$

where vectors $X$ and $Y$, respectively, represent the first and second column of the matrix M. There are multiple features that can be extracted from the matrix $\mathbf{M}$. These features will be used in developing the proposed detection algorithm. This algorithm is discussed in more detail in the following subsections. It consists of three main parts which, are Data Processing, Covariance Computation, and Fault Signatures. The first operation is to send the raw data (three-phase currents) to the Data Processing module where the data is normalized and filtered. The second operation is Covariance Computation where the absolute value of the covariance and it's mean of the normalized data is computed. In the third module, Fault Signatures, a look-up table is presented, where the mean of the absolute covariance signal along with current values determines and localizes an open-switch fault. 


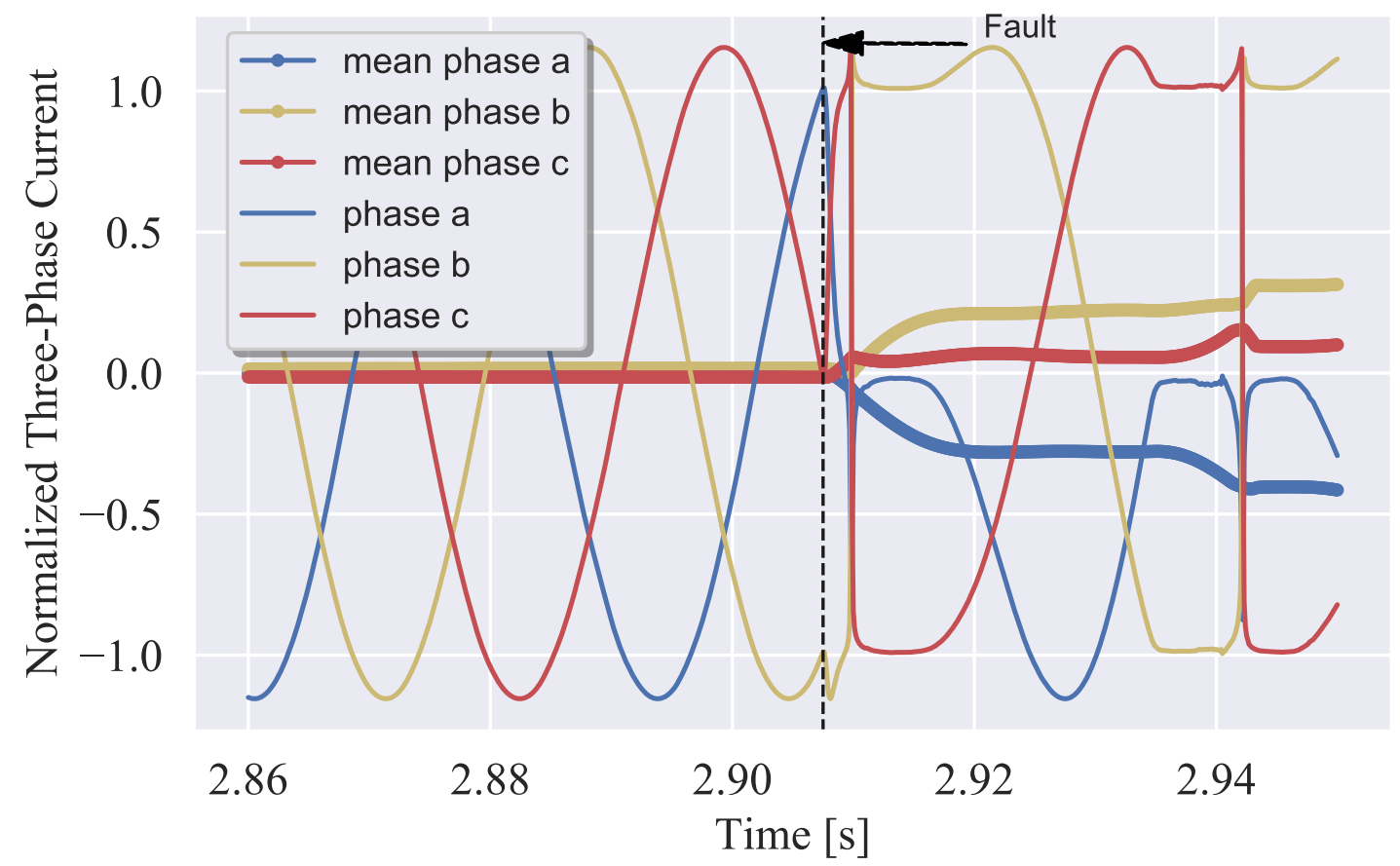

Figure 4.5: Experimental: the normalized three-phase current during pre- and postfault at switch $S_{1}$

\subsubsection{Data Processing}

In this module, the three-phase currents are sampled and the matrix $M$ is constructed. Each column of the matrix $\mathrm{M}$ is normalized by making its mean equal to zero and its standard deviation equal to one. To illustrate the effect of an open-switch fault at switch $S_{1}$, a simulation has been carried out. Figure 4.5 depicts a normalized threephase current during both pre- and post-fault periods. These currents are normalized. It can be noted that since the fault happened at $S 1$, there is no positive current at phase $a$. The fault-angle in this fault is about 70 degrees. 


\subsubsection{Covariance Computation}

One of the important features for a faster fault detection is the absolute value of the covariance matrix $\mathbf{M}$. For a given matrix $\mathbf{M} \in \mathbb{R}^{\mathbf{n} \times \mathbf{2}}$, the formula used for calculating the covariance is obtained as

$$
\operatorname{COV}(\mathbf{M})=\frac{\sum_{i=1}^{n}\left(X_{i}-\bar{x}\right)\left(Y_{i}-\bar{y}\right)}{n-1}
$$

where $\bar{x}$ and $\bar{y}$ denote the instantaneous means of $X$ and $Y$, respectively, and $n$ represents the number of observations in the matrix. The covariance of the matrix M presented in Equation (4.18) has three observations and two variables. Therefore, the covariance of this matrix yields a two-by-two matrix and it is represented as

$$
\operatorname{COV}(\mathbf{M})=\left[\begin{array}{ll}
C_{X X} & C_{X Y} \\
C_{Y X} & C_{Y Y}
\end{array}\right]
$$

Since the input signals in matrix $\mathbf{M}$ are normalized, the co-variance of each observation vector by itself always equals one. Therefore, the $C_{X X}$ and $C_{Y Y}$ are always one and $C_{X Y}$ and $C_{Y X}$, in a healthy state, are always equal to

$$
C_{X Y}=C_{Y X}=A \cos \left(3 \omega t+\frac{\pi}{3}\right)
$$

where $\omega$ is the synchronous angular velocity of the motor currents and $A$ represents the magnitude of the sinusoid. This magnitude is 1 due to normalization. The absolute value of the $\operatorname{COV}(\mathbf{M})$ is then 


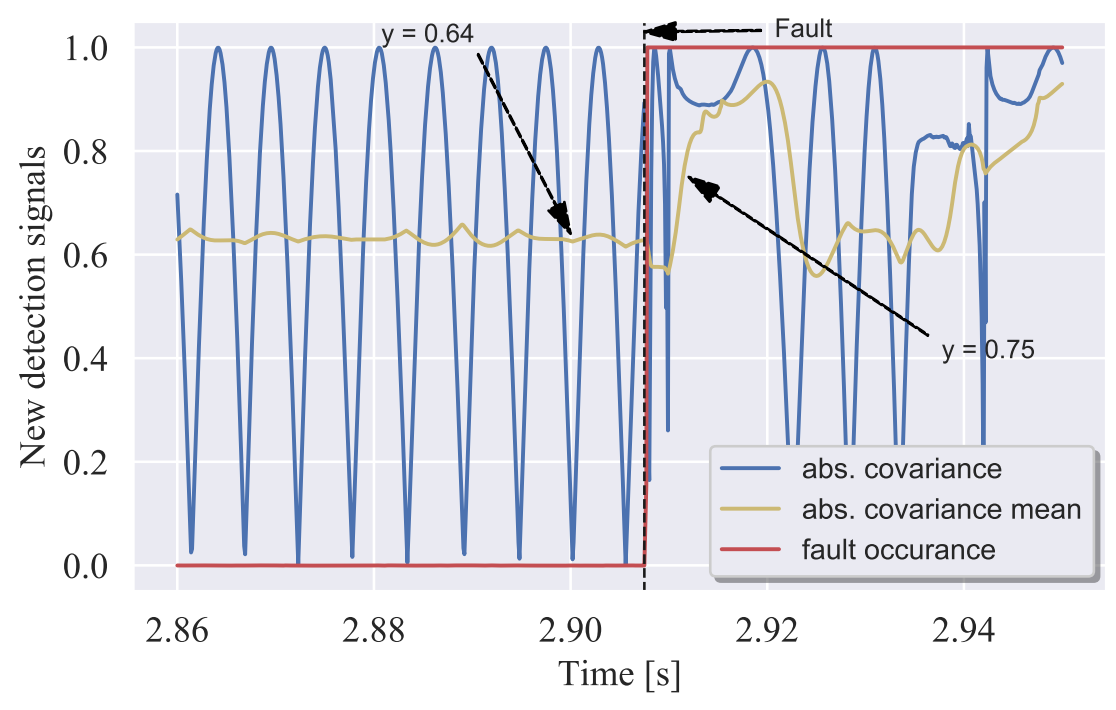

Figure 4.6: Experimental: co-variance value and its mean during pre- and post-fault

$$
|\operatorname{COV}(\mathbf{M})|=\left[\begin{array}{cc}
1 & \left|\cos \left(3 \omega t+\frac{\pi}{3}\right)\right| \\
\left|\cos \left(3 \omega t+\frac{\pi}{3}\right)\right| & 1
\end{array}\right]
$$

It can be noted that $C_{X Y}$ is a sinusoid and varies three times faster than the operating frequency. By taking the absolute value of the matrix, $\left|C_{X Y}\right|$ will be a full wave sinusoid with a frequency six times the operating frequency. The mean of the $\left|C_{X Y}\right|$ is calculated to be $\left|\bar{C}_{X Y}\right|=(2 / \pi) A \approx 0.636$. Figure 4.6 presents $\left|C_{x y}\right|$ with its mean, $\left|\bar{C}_{X Y}\right|$. Once the fault occurs, the covariance value $\left|C_{x y}\right|$ will have a higher value and its mean increases to about 0.8 , as shown in Fig 4.6. The value of $\left|C_{X Y}\right|$ does not vary in value during a transient, making it a very reliable indication of the occurrence of a fault. There are multiple signals that are required for this algorithm to identify the fault and localize the faulty switch. These signals are $\left|\bar{C}_{X Y}\right|$, the instantaneous and mean values of $i_{a}(t), i_{b}(t)$, and $i_{c}(t)$ and they are used in the algorithm look-up table. 
Table 4.2: Fault localization look-up table

\begin{tabular}{|l|l|l|l|l|}
\hline \multirow{5}{*}{$\left|C_{X Y}\right|>0.75$} & $\left|i_{a}\right|<0.2$ & $\left(\left|i_{b}\right|>0.9\right) \&\left(\left|i_{c}\right|>0.9\right)$ & $i_{a, a v g}<-0.1$ & Fault $S_{1}$ \\
\cline { 4 - 5 } & & & $i_{a, a v g}>0.1$ & Fault $S_{2}$ \\
\cline { 4 - 5 } & $\left|i_{b}\right|<0.2$ & $\left(\left|i_{a}\right|>0.9\right) \&\left(\left|i_{c}\right|>0.9\right)$ & $i_{b, a v g}<-0.1$ & Fault $S_{3}$ \\
\cline { 4 - 5 } & & & $i_{b, a v g}>0.1$ & Fault $S_{4}$ \\
\cline { 4 - 5 } & $\left|i_{c}\right|<0.2$ & $\left(\left|i_{a}\right|>0.9\right) \&\left(\left|i_{b}\right|>0.9\right)$ & $i_{c, a v g}<-0.1$ & Fault $S_{5}$ \\
\cline { 3 - 5 } & & $i_{c, a v g}>0.1$ & Fault $S_{6}$ \\
\hline
\end{tabular}

\subsubsection{Fault Signatures}

In the previous computation module, the response of the three-phase current and the absolute covariance mean were shown during the post-fault period for switch $S_{1}$. Two of the features that indicate a fault in the system are $\left|\bar{C}_{X Y}\right|$ and magnitude of the faulty phase. For any open-switch fault $\left|\bar{C}_{X Y}\right|$ becomes greater than 0.8 and the faulty phase current is around zero, as shown in Fig. 4.5. Instead of using a zero value, a band of \pm 0.2 (PU) is chosen to avoid a false flag due to a rounding problem in the computation or noise in the system. Since $i_{a}=0$ in the post-fault period, the relationship between $i_{b}$ and $i_{c}$ will always be $i_{b}=-i_{c}$. Also, values of $i_{c}$ and $i_{b}$ are either greater than 0.9 or smaller than -0.9 . Therefore, using a very fast indicator of the absolute co-variance along with values of the normalized current, a fast fault detection is possible.

These responses are different for each switch fault. Since multiple open-switch fault scenarios exist, a look-up table containing multiple signatures is necessary. Table 4.2 illustrates all of the possible faults in the inverter and their signatures.

In Figure 4.7(c), the detection time for the proposed method and the Park vector method $[28,29]$ are compared. To compare these two methods, multiple faults at different fault angles and at different operating speeds have been introduced in the three-phase inverter and the performance of the two algorithms are evaluated. The 

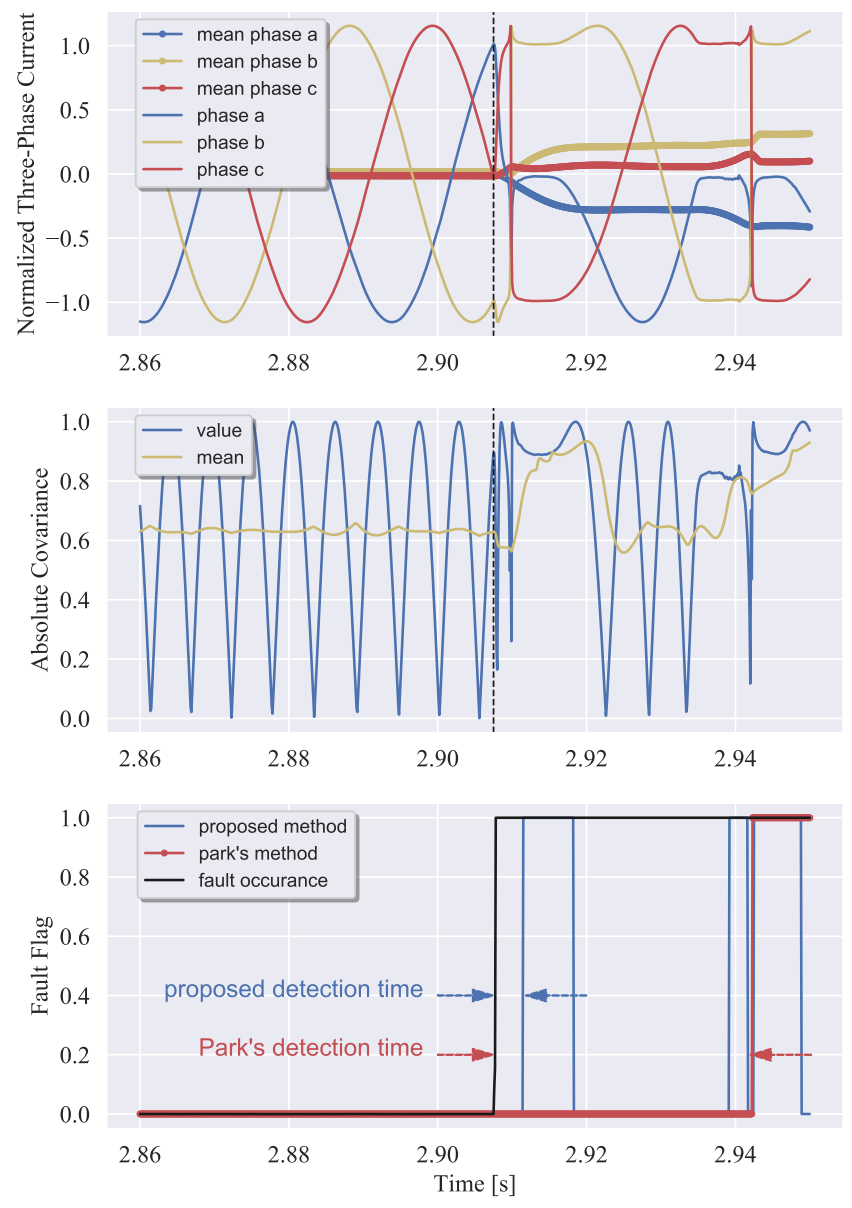

Figure 4.7: Experimental results: (a) three-phase currents and their mean; (b) absolute value of the co-variance; (c) fault flag for park vector and the proposed vector method

results of these comparisons are provided in the next section.

\subsection{Results}

\subsubsection{Simulation Results}

The overall system of the three-phase inverter and the load model have been simulated in order to verify the effectiveness and functionality of the proposed algorithm, using 
SIMULINK. The simulation models consist of speed and torque control commands, gate control signals using space-vector pulse width modulation (SVPWM), and a three-phase induction motor. A 10-hp motor is modeled as the load. Figure 4.7(a) illustrates the normalized three-phase currents and their mean. During the simulation an open-switch fault is applied to the inverter by keeping the switch gate signal zero, at switch $S 1$, and keeping a normal operation of the complementary switch $S 2$. A fault is introduced at $t=2.907 \mathrm{~s}$ as shown in 4.7 (a).

Figure $4.7(\mathrm{~b})$ shows the absolute covariance value and its mean, $\left|\bar{C}_{X Y}\right|$. The proposed method uses $\left|\bar{C}_{X Y}\right|$ to determine whether a fault is present in the system or not. It can be observed that the average of the $\left|C_{X Y}\right|$ is around 0.636 and as soon as the fault occurs, the mean reaches 0.8. In Fig. 4.7(c), the detection time of the two methods, the Park vector method $[28,29]$ and the proposed method, have been compared. The results were obtained for an operating frequency of $f=30 \mathrm{~Hz}$. It is evident that the proposed method detects the fault sooner than the Park averaged method. To evaluate this algorithm thoroughly, multiple faults with different fault angles were tested from $0^{\circ}$ to $360^{\circ}$. A total of sixteen fault angles were chosen as our test cases, as shown in Fig. 4.8. Four operating frequencies of $15 \mathrm{~Hz}, 30 \mathrm{~Hz}, 45 \mathrm{~Hz}$, and $60 \mathrm{~Hz}$ were chosen as the operating frequency in these simulations. Therefore, sixteen simulations for each operating speed yielded a total of 64 simulations which have been carried out for each switch. It is worth noting that the simulation results for all of the switches are similar and, therefore, only the results for $S 1$ suffice. Figure 4.8 depicts the detection time obtained for the simulations that were carried out for the different fault angles at multiple operating frequencies. In all of the cases, the proposed method has a superior performance. Between fault angles of $60^{\circ}$ and $120^{\circ}$, the detection time is much lower than the Park vector method. 

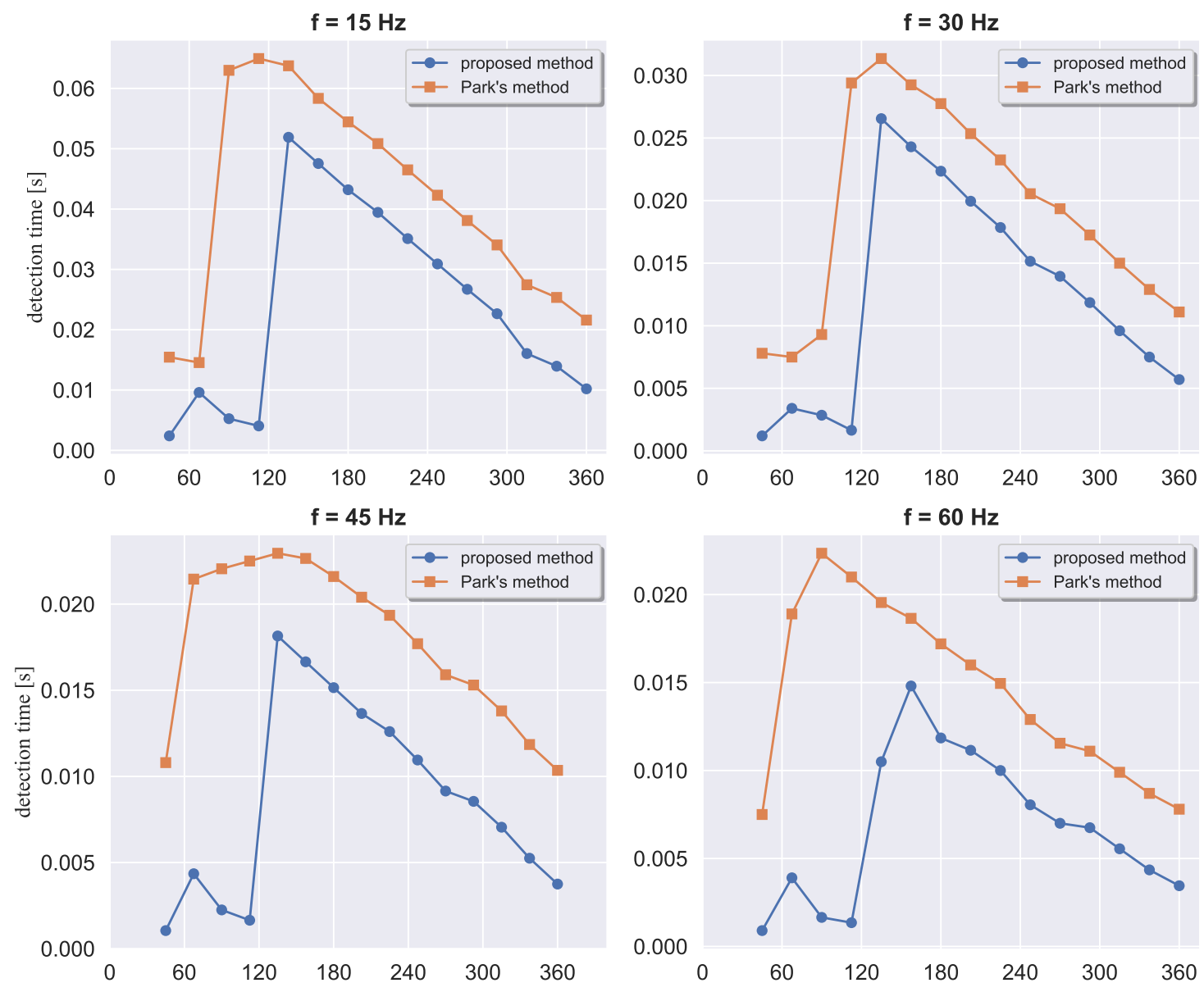

Figure 4.8: Simulation results of the detection time for different fault-angle at different operating frequencies at switch $S 1$

\subsubsection{Experimental Results}

In these experiments, two motors are coupled to each other. The 130 Watt, four pole, three-phase AC motor is used to obtain the desired shaft speed and the 250 Watt, 42 Volt DC motor is used to apply a load torque to the shaft. A three-phase voltagesource inverter is required to carry out the experiment. A three-phase inverter, 500 VDC with maximum current output of 21 Arms per phase has been developed at the Power Lab at Boise State University. This inverter has six isolated and independent 

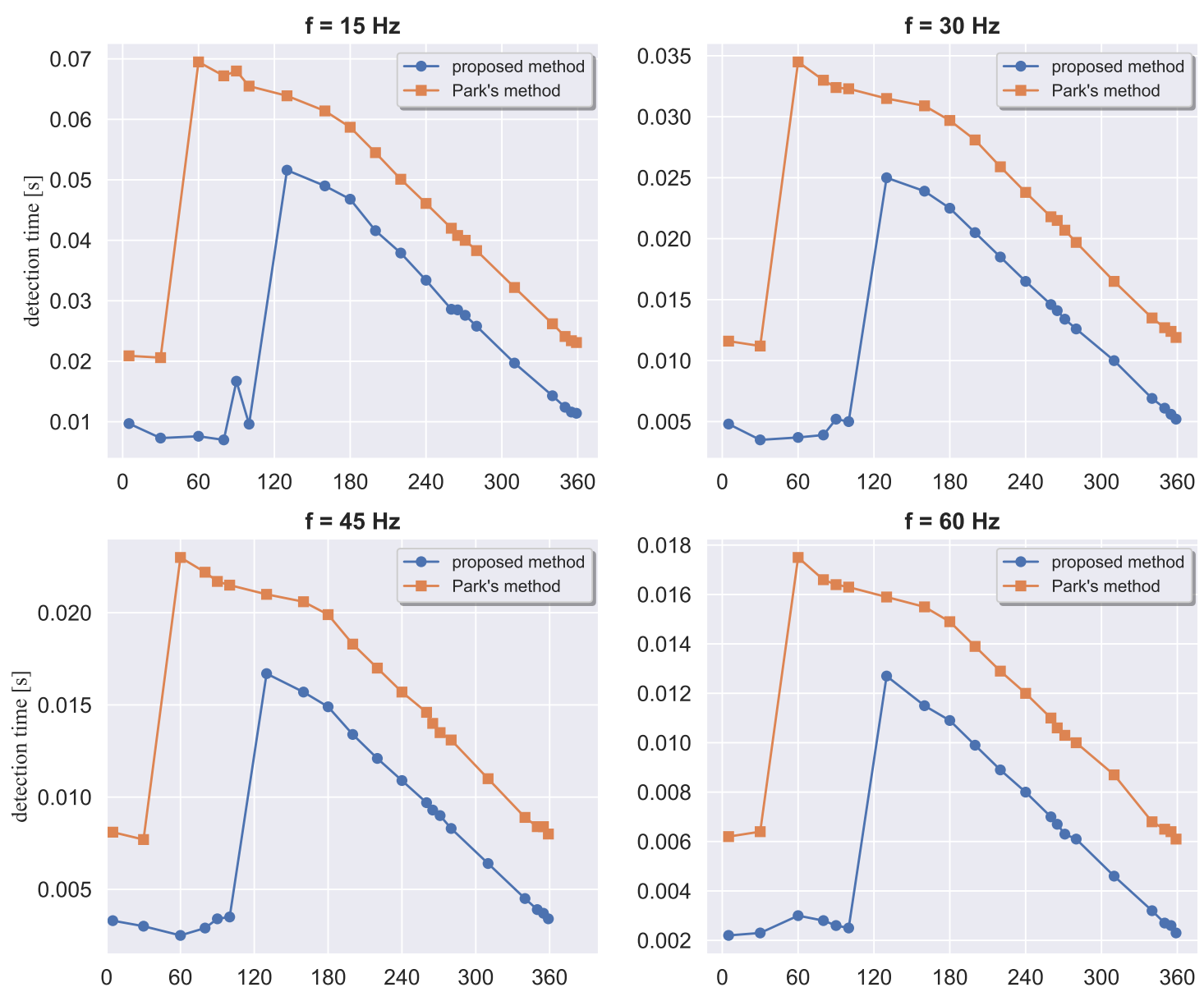

Figure 4.9: Experimental results of the detection time for different firing angle at different operating frequencies at switch $S 1$

MOSFETs. Each of the switches can be controlled independently. The main feature of this board, compared to the commercially-available inverters in the Power Lab, is that all of its switches can be controlled independently and the capacitor connected to the bootstrap must be stable for the duration of the short-circuited or open-switch fault. This feature allows turning off one of the switches at any given time in order to evaluate the developed detection algorithm.

A high-frequency three-phase gate driver of IR2136 was used for applying gatesource voltages of the MOSFETs. In designing the gate driver, specifically for 


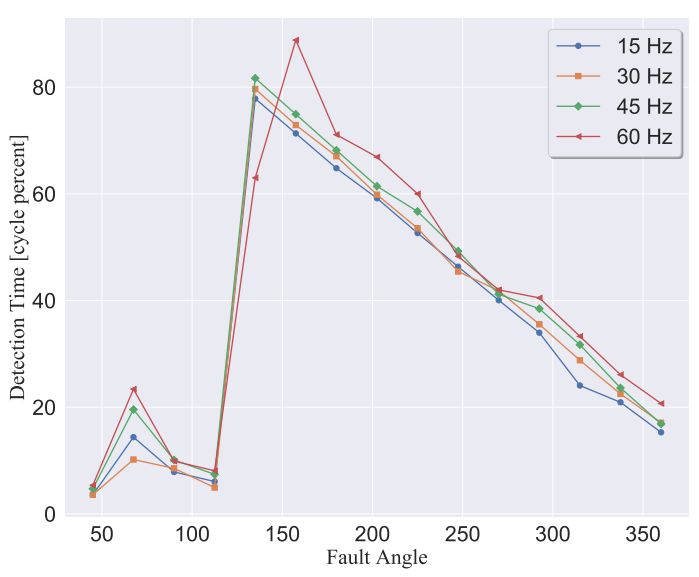

(a) Simulation results of the detection time

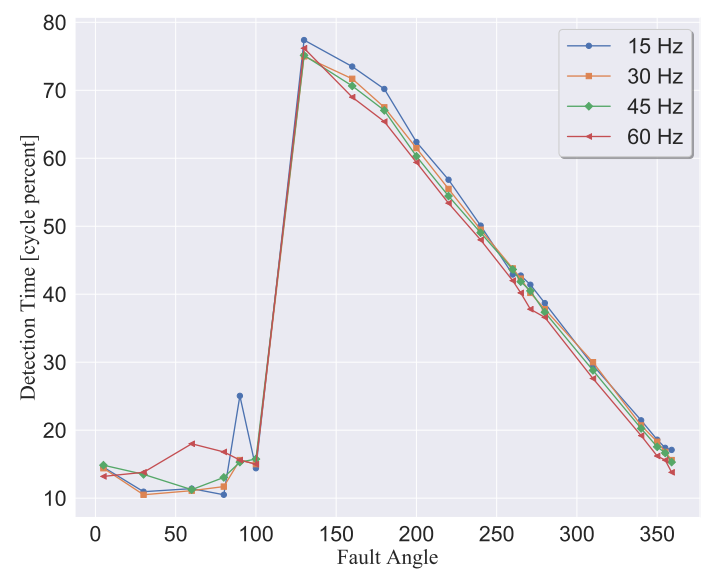

(b) Experimental results of the detection time

Figure 4.10: Detection time in percentage of a cycle for different firing angle at different operating frequencies at switch $S 1$

studying faults, the size of $C_{b s}$, capacitor between $V_{B}$ and $V_{S}$ in IR2136, is important. The voltage across $C_{b s}$ must be stable and constant for the duration of the fault. In this design, the capacitor size of $220 \mu F$ has been used. This value has shown enough stability to keep the voltage across $C_{b s}$ stable and constant for the duration of the fault.

The control system of the three-phase induction motor has been implemented on a Field-Programmable Gate Array (FPGA) where the SVPWM signals are generated. The controller in the FPGA uses the appropriate PWM signals to control the speed of the motor by applying the proper voltages from the three-phase inverter. The Hall-effect sensors of the board measure the phase currents of the load. In order to be able to apply the fault at the desired fault angle, the three-phase currents in $a b c$-coordinate system are converted to the $\alpha \beta$-coordinate system and obtain the angle. Once the target angle is reached, the fault signal is issued. The amount of time required for the detection algorithm for each target angle is calculated and logged. In 
these experiments, dSPACE 1104 is used for capturing and storing the three-phase currents and fault related signals.

Overall the simulation results match the experimental result very well. The detection time of the proposed algorithm is much lower than the conventional method, especially at fault angles between from 60 to 120 degrees, as shown in Figures 4.8 and 4.9. In Figure 4.10, the simulation and experimental results at different frequencies are presented collectively using a different y-axis variable. In these two figures, the fault detection time has been scaled as a percentage of a full period which has the advantage of being independent of the study frequency. This figure shows that all of the previous graphs at different frequencies are now almost superimposed on each other, except for some minor discrepancies.

\subsection{Extension to a Five-Phase Drive System}

In the previous section, the absolute covariance (A-COV) method was applied to a three-phase induction motor drive, where the functionality and performance of the algorithm was verified and compared to the Park vector method. In this section, the A-COV method is applied to a five-phase motor for the detection of an open-switch fault. A detailed analysis of the five-phase motor, similar to Chapter 2, can be found in [8]. Utilizing a five-phase model, several simulations have been carried out to investigate the performance of the A-COV method. The simulation results show that a strong fault signature signal can be achieved using the A-COV method, which is a reliable indication of an open-switch fault. In this section, the simulation results of different fault angles for multiple operating frequencies are presented. In the following, this strong fault indicator is used along with the instantaneous and mean values of 
the phases currents to detect and to localize an open-switch fault.

\subsubsection{A-COV Methodology for a Five-Phase Motor}

In a healthy system, the instantaneous values of the currents in a five-phase permanent magnet synchronous motor (PMSM) can be expressed as

$$
\begin{aligned}
& i_{a}(t)=\Re e\left\{I_{m} \angle(\omega t)\right\} \\
& i_{b}(t)=\Re e\left\{I_{m} \angle\left(\omega t-\frac{2 \pi}{5}\right)\right\} \\
& i_{c}(t)=\Re e\left\{I_{m} \angle\left(\omega t-\frac{4 \pi}{5}\right)\right\} \\
& i_{d}(t)=\Re e\left\{I_{m} \angle\left(\omega t+\frac{4 \pi}{5}\right)\right\} \\
& i_{e}(t)=\Re e\left\{I_{m} \angle\left(\omega t+\frac{2 \pi}{5}\right)\right\}
\end{aligned}
$$

where $\omega$ is the angular frequency of the stator currents. The stator currents in the $\alpha \beta$-coordinates can be calculated using

$$
\left[\begin{array}{c}
i_{\alpha} \\
i_{\beta}
\end{array}\right]=\frac{2}{5}\left[\begin{array}{ccccc}
1 & \cos \left(\frac{2 \pi}{5}\right) & \cos \left(\frac{4 \pi}{5}\right) & \cos \left(\frac{6 \pi}{5}\right) & \cos \left(\frac{8 \pi}{5}\right) \\
1 & -\sin \left(\frac{2 \pi}{5}\right) & -\sin \left(\frac{4 \pi}{5}\right) & -\sin \left(\frac{6 \pi}{5}\right) & -\sin \left(\frac{8 \pi}{5}\right)
\end{array}\right]\left[\begin{array}{c}
i_{a} \\
i_{b} \\
i_{c} \\
i_{d} \\
i_{e}
\end{array}\right]
$$

To apply a fault at a specific fault angle, these five-phase currents in the abcdecoordinates are converted to $\alpha \beta$-coordinates using (4.16). The phase angle of the space vector can be calculated using $\tan ^{-1}\left(i_{\beta} / i_{\alpha}\right)$. An open-switch fault can be applied at any specific fault angle. In a five-phase, five-wire motor system operation, 
the following equation always holds:

$$
i_{a}(t)+i_{b}(t)+i_{c}(t)+i_{d}(t)+i_{e}(t)=0
$$

The captured data sets from the five-phase currents are utilized to create a new detection feature which is constructed in a similar fashion to the three-phase case. Hence, the feature vector is constructed by multiplying two of the phases together. The matrix, $\mathbf{M} \in \mathbb{R}^{\mathbf{2} \times 5}$, has two columns where the first column of this matrix consists of all of the instantaneous values of the five-phase current, $i_{a}(t), i_{b}(t), i_{c}(t), i_{d}(t)$, and $i_{e}(t)$. The second column consists of the multiplication of two adjacent phase currents together as $i_{a}(t) i_{b}(t), i_{b}(t) i_{c}(t), i_{c}(t) i_{d}(t), i_{d}(t) i_{e}(t)$, and $i_{e}(t) i_{a}(t)$. Since these currents are multiplied by each other, the second column constructs data points with a frequency higher than the frequency of the original currents, as in the case of the three-phase motor. In matrix form, the matrix $\mathbf{V}$, is represented as

$$
\mathbf{V}=\left[\begin{array}{cc}
i_{a}(t) & i_{a}(t) i_{b}(t) \\
i_{b}(t) & i_{b}(t) i_{c}(t) \\
i_{c}(t) & i_{c}(t) i_{d}(t) \\
i_{d}(t) & i_{d}(t) i_{e}(t) \\
i_{e}(t) & i_{e}(t) i_{a}(t)
\end{array}\right]
$$

and, to alleviate the notation, we write it

$$
\mathbf{V}=\left[\begin{array}{ll}
X & Y
\end{array}\right]
$$

where vectors $X$ and $Y$, respectively, represent the first and second column of the 
matrix $\mathbf{V}$. There are multiple features that can be extracted from matrix $\mathbf{V}$. In this work, a covariance feature is constructed as a two-by-two matrix by

$$
\operatorname{COV}(\mathbf{V})=\left[\begin{array}{ll}
C_{X X} & C_{X Y} \\
C_{Y X} & C_{Y Y}
\end{array}\right]
$$

The covariance of matrix $\mathbf{V}$ has been defined in (4.7). Since the input signals in this matrix are normalized, the covariance of each observation vector by itself always equals one. Therefore, unlike in a three-phase system, the $C_{X X}$ and $C_{Y Y}$ are always one and $\left|C_{X Y}\right|$ and $\left|C_{Y X}\right|$ are always equal to zero. Hence,

$$
\left|C_{X Y}\right|=\left|C_{Y X}\right|=0
$$

when there is a fault, the value of $\left|C_{X Y}\right|$ approaches 1. Figure 4.11 illustrates the behavior of $\left|C_{X Y}\right|$ for pre- and post-fault.

It is worth noting that, once a fault happens, the feature $\left|C_{X Y}\right|$, has a double-hump shape with one peak smaller than the other. A more in-depth space-vector analysis of the currents may explain the double-hump shape. Even though the smaller hump would be a good indicator of a fault, the bigger hump peak is used as the main fault signature in this work.

The phase current of the corresponding faulty switch is zero due to a switch malfunctioning. For instance, in case of an open-switch fault at switch $S_{3}$, the $i_{b}=0$ during the positive cycle and Eq. (4.17) becomes

$$
i_{a}+i_{c}+i_{d}+i_{e}=0
$$



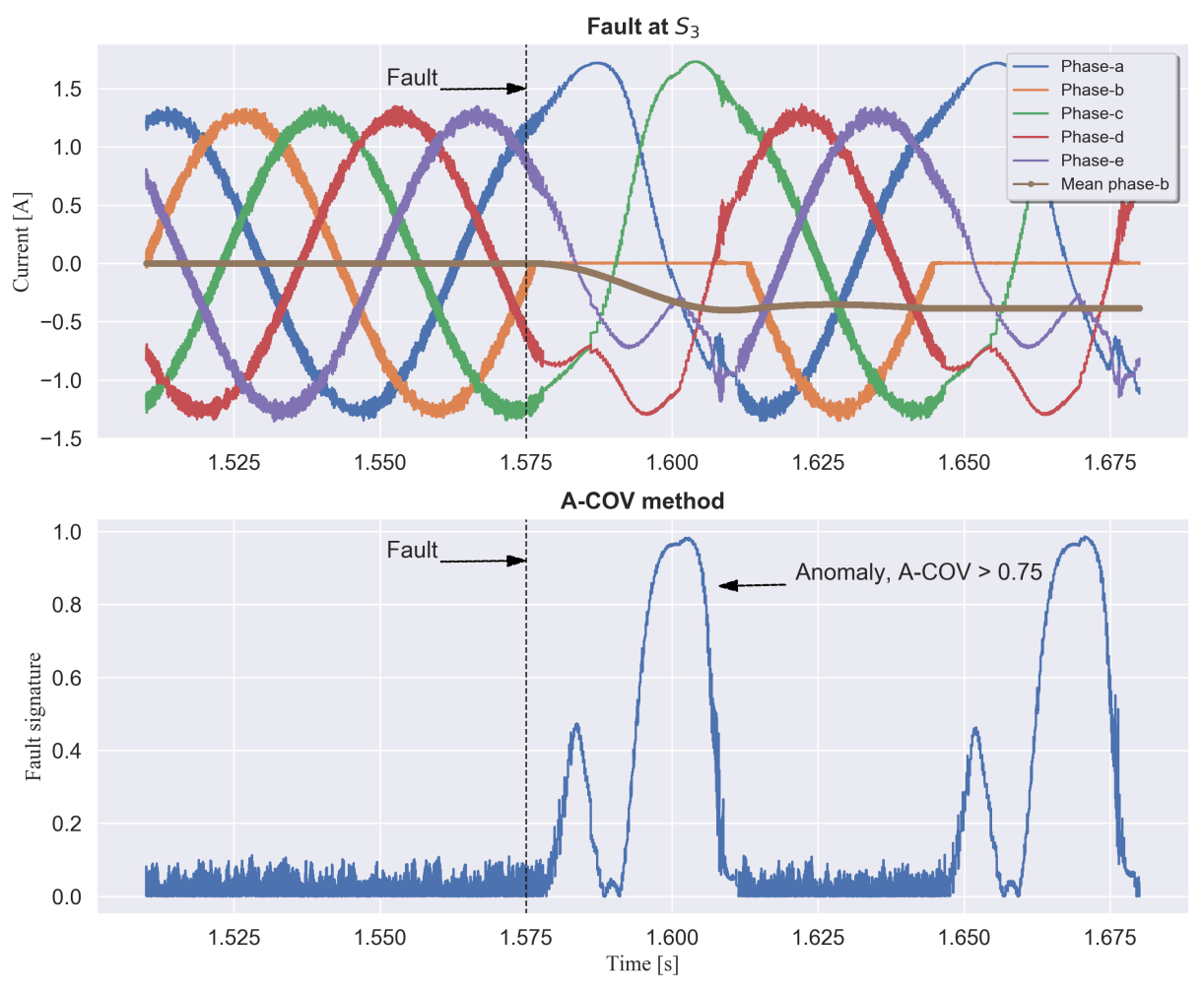

Figure 4.11: Simulation of the $C_{X Y}$ and five-phase motor currents during pre- and post-fault conditions

From Figure 4.11, it can be observed that, during the fault, the phase-c current is positive and the phase currents $i_{a}, i_{d}$, and $i_{e}$ are negative. Since the current of phase- $b$ is zero during a positive half cycle, the mean of phase- $b$ becomes negative. Taken together, these fault signatures are used to detect an open-switch fault $S_{3}$. All of the signal condition used for the open-switch fault detection of the $S_{3}$ are shown in Fig. 4.12.

By simulating an open-switch fault for each switch, the responses of the remaining four phase currents can be documented and stored in a look-up table. This look-up table can be used to determine and localize the faulty switch. Table 4.3 presents the behavior of the remaining four-phase currents. The last column of Table 4.3, shows 


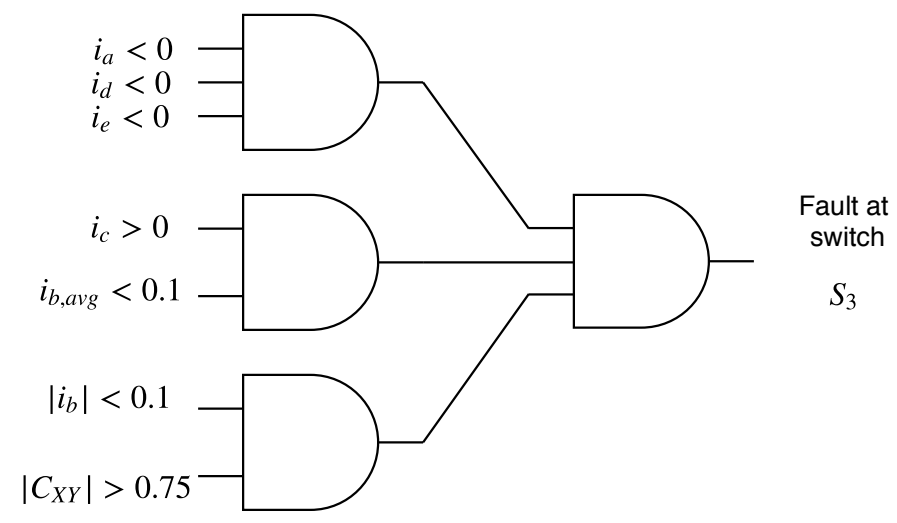

Figure 4.12: Detection logic gates for an open-switch fault at $S_{3}$

the corresponding switch faults.

Table 4.3: Fault localization look-up table for a five-phase inverter

\begin{tabular}{|c|l|l|l|l|l|}
\hline \multirow{5}{*}{$\left|i_{a}\right|<0.1$} & $i_{a, a v g}<-0.1$ & $i_{b}>0$ & $\left(i_{c} \& i_{d} \& i_{e}\right)<0$ & Fault $S_{1}$ \\
\cline { 2 - 6 } & $i_{a, a v g}>0.1$ & $i_{b}<0$ & $\left(i_{c} \& i_{d} \& i_{e}\right)>0$ & Fault $S_{2}$ \\
\cline { 2 - 6 } & \multirow{2}{*}{$\left|i_{b}\right|<0.1$} & $i_{b, a v g}<-0.1$ & $i_{c}>0$ & $\left(i_{d} \& i_{e} \& i_{a}\right)<0$ & Fault $S_{3}$ \\
\cline { 2 - 6 } & $i_{b, a v g}>0.1$ & $i_{c}<0$ & $\left(i_{d} \& i_{e} \& i_{a}\right)>0$ & Fault $S_{4}$ \\
\cline { 2 - 6 } & \multirow{2}{*}{$\left|i_{c}\right|<0.1$} & $i_{c, a v g}<-0.1$ & $i_{d}>0$ & $\left(i_{a} \& i_{b} \& i_{e}\right)<0$ & Fault $S_{5}$ \\
\cline { 2 - 6 } & $i_{c, a v g}>0.1$ & $i_{d}<0$ & $\left(i_{a} \& i_{b} \& i_{e}\right)>0$ & Fault $S_{6}$ \\
\cline { 2 - 6 } & \multirow{2}{*}{$\left|i_{d}\right|<0.1$} & $i_{d, a v g}<-0.1$ & $i_{e}>0$ & $\left(i_{a} \& i_{b} \& i_{c}\right)<0$ & Fault $S_{7}$ \\
\cline { 2 - 6 } & $i_{d, a v g}>0.1$ & $i_{e}<0$ & $\left(i_{a} \& i_{b} \& i_{c}\right)>0$ & Fault $S_{8}$ \\
\cline { 2 - 6 } & \multirow{2}{*}{$\left|i_{e}\right|<0.1$} & $i_{e, a v g}<-0.1$ & $i_{a}>0$ & $\left(i_{b} \& i_{c} \& i_{d}\right)<0$ & Fault $S_{9}$ \\
\cline { 3 - 6 } & $i_{e, a v g}>0.1$ & $i_{a}<0$ & $\left(i_{b} \& i_{c} \& i_{d}\right)>0$ & Fault $S_{10}$ \\
\hline
\end{tabular}

The second column in this table is the absolute value of the corresponding phase current. During a fault, this current becomes zero, due to the quantization error and noise, a threshold value of 0.1 instead of zero is used, as in the case of the three-phase system. 


\subsubsection{Simulation Results}

A five-phase permanent-magnet synchronous machine (PMSM) has been modeled in SIMULINK. The motor parameters are $R_{s}=0.12 \Omega, L_{\ell s}=0.00135 \mathrm{H}, J=$ $0.002 \mathrm{~kg} . \mathrm{m}^{2}$, Friction $=0.02$ N.m.s, and $p=4$ poles $/$ phase. The sampling time of $T_{s}=2 \mu \mathrm{s}$ is used. In the following section, two aspects of detection timing and reliability tests are presented.

\section{Detection Timing Report}

As in the case of the three-phase motor, at every $\frac{\pi}{16}$ fault angle, a fault is introduced in the system and the detection time is recorded. The test cases have been designed to carry out tests at multiple frequencies of operation. These frequencies are $15 \mathrm{~Hz}$, $30 \mathrm{~Hz}, 45 \mathrm{~Hz}$, and $60 \mathrm{~Hz}$. The results shows a similar pattern when compared to the results obtained for a three-phase motor. There is a sudden increase in detection time around a fault angle of $120^{\circ}$. Also, the higher the frequency of the operation, the faster the detection time, as expected.

\section{Reliability Tests Report}

In this subsection, the reliability of the algorithm during a transient state where a mechanical load is applied to the five-phase motor is tested. During these simulations, the goal is to test the algorithm for false positives. Hence, no switch fault is applied to the five-phase motor. In this experiment, Figure 4.14 shows a complete simulation results, where the five-phase currents, the torque applied to the motor, the A-COV signal, and the fault flag signal are illustrated. These simulations are done by keeping the frequency of the operation constant at $15 \mathrm{~Hz}, 30 \mathrm{~Hz}, 45 \mathrm{~Hz}$, and $60 \mathrm{~Hz}$. The 

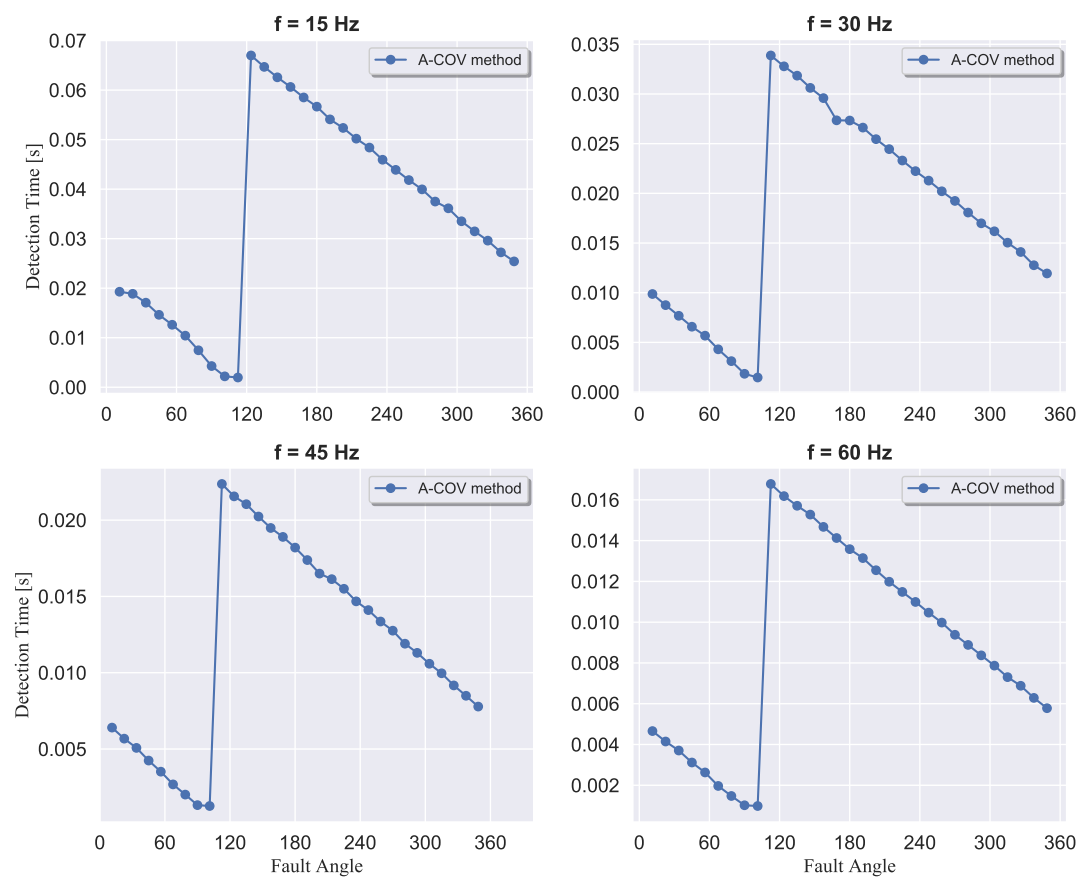

Figure 4.13: Simulation results of detection time for different frequency for a fivephase motor

torque applied is located in the second column of Fig. 4.14. The random values of the torque are within $[-11,11]$ N.m interval. There are eight torque values which are chosen randomly and these values are applied to the five-phase motor at eight random times during the simulation. There are four more simulation results available in Appendix ??. The following MATLAB script is used to generate the values for torque and time.

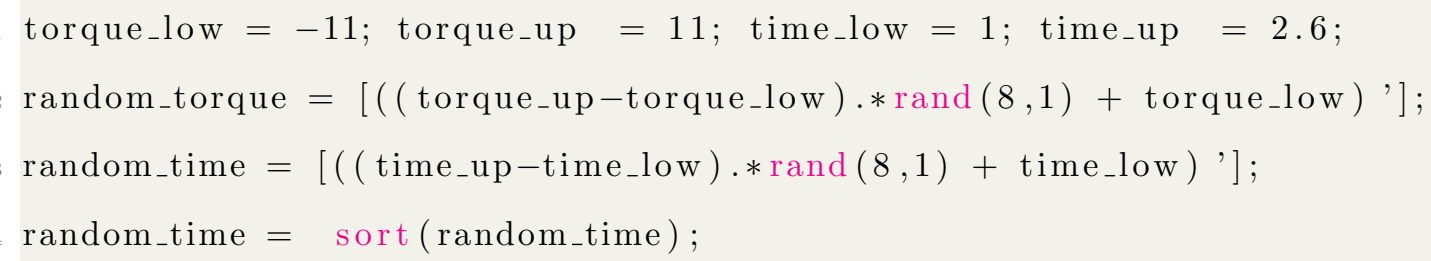

The rand function is uniformly distributed in the interval $(0,1)$. The third column is the A-COV signal. In all of the simulations, the A-COV signal value stays zero or is 

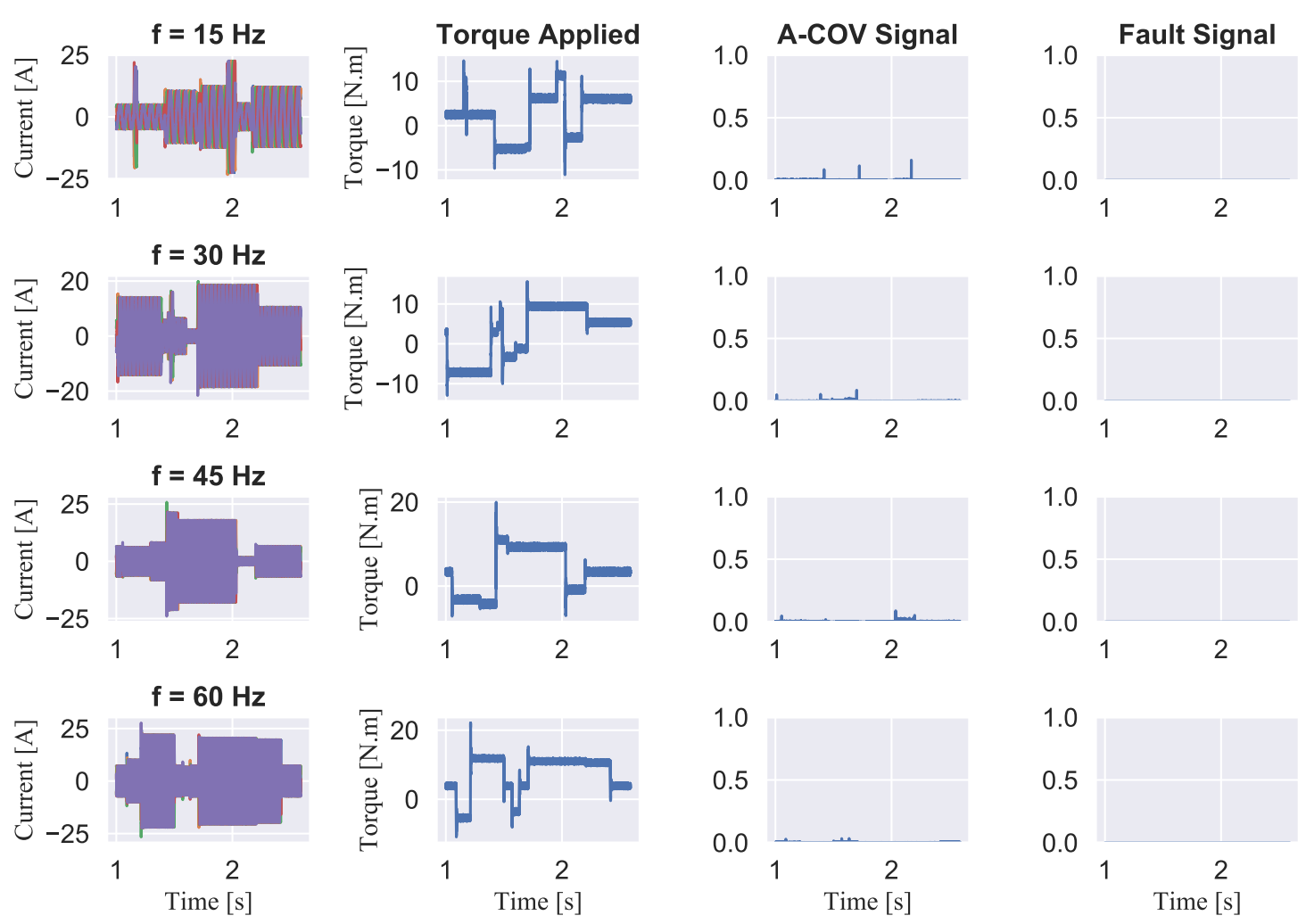

Figure 4.14: Simulation of A-COV and fault signal with random torque command at random times

relatively small compared to the anomaly value of 0.75 .

\section{Precision, Recall, F-score}

The criteria for evaluating the performance of the algorithm is defined as Precision, Recall, and F-Score. In the analysis of detection or classification, F-Score is used to evaluate the accuracy of the test results. To compute an F-score value, two parameters of precision and recall are required.

Table 4.4 demonstrates the parameters used for calculating the Precision and Recall. For instance, if our test results predict Good for a Good Sample (healthy signal), it is called True Positive and if the test results predict bad for a bad signal, it 
is called True Negative. Precision is the number of correct positive results divided by

Table 4.4: Test and evaluation parameter definition

\begin{tabular}{|c|c|c|}
\hline & Good Sample & Bad Sample \\
\hline Predicted Good & True Positive & False Positive \\
\hline Predicted Bad & False Negative & True Negative \\
\hline
\end{tabular}

the number of all positive results detected (True Positive and False Positive Samples), and Recall is the number of correct positive results divided by the number of positive results that should have been returned. Therefore, definitions used for Precision, Recall, and F-Score are as follows:

$$
\begin{aligned}
\text { Precision } & =\frac{\text { True Positive }}{\text { True Positive }+ \text { False Positive }} \\
\text { Recall } & =\frac{\text { True Positive }}{\text { True Positive }+ \text { False Negative }}
\end{aligned}
$$

Then, F-score is expressed as

$$
\text { F-Score }=2 \times \frac{\text { Precision } \times \text { Recall }}{\text { Precision }+ \text { Recall }} \text {. }
$$

There are seventy simulations that have been carried out for each of the four operating frequencies. Therefore, the 280 simulations and 8 different torque values for each simulation, then, the total of 2240 data sets have been completed in order to analyze the detection precision and recall. The precision and accuracy results for these simulations are $100 \%$. Therefore, the F-score is $100 \%$. 


\subsection{Conclusion}

In this work, a new detection algorithm has been introduced and explained. The functionality of this method is verified by an experimental setup for various fault angles and different operating speeds of a three-phase induction motor. The performance of this new algorithm has been compared to another non-invasive method described in the literature. The proposed algorithm has a superior performance in terms of detection time. This method, as mentioned earlier, is a non-invasive method and can be implemented easily on a microcontroller for the fault detection in the motor drive. The proposed method has been applied to five-phase PMSM and it was shown that this method can also be used in the five-phase PMSM. 


\section{CHAPTER 5}

\section{DESIGN IMPLEMENTATION ON SOC}

In this chapter, a new hardware/software design and implementation of an Induction Motor (IM) drive control topology is presented. Power electronic applications such as three-phase inverters require an accurate switching frequency for the gating signals of the switches. This design uses a System-on-Chip ( $\mathrm{SoC})$ approach implemented on a Field Programmable Gate Array (FPGA). The SoC includes the FPGA fabric and an on-chip processor. The on-chip processor is used for high-level programing, while the FPGA programmable fabric is used to create precise gating signals and fast parallel calculations for a three-phase inverter. These signals are generated in the hardware side of the design. Floating-point calculations and the control flow of the whole design are managed by the SoC. This method is suitable for any power electronic application where precise gating signals are required.

The vast number of inputs-outputs (IOs) in the FPGA allows designers to generate several and various numbers of gating signals, since in microcontrollers the number of PWM signals and their timings are limited. In this work, the SoC platform for power electronic applications is introduced and published in [38] and also implemented for the verification of the detection algorithm introduced in Chapter 4. The methodology used in this novel solution is explained and experimental results are presented. 


\subsection{Introduction}

Alternating-Current (AC) motors are widely used in industry. Their high performance allows for an accurate control of electric machines. The current trend in industry requires monitoring the performance and status of the motor through the use of popular networking protocols such as Controller Area Network (CAN) and Ethernet. Controlling a motor drive using FPGAs has become a common trend for many years. In some recent research work [39-41], the multiple benefits of using FPGAs in motor drive applications have been enumerated. Incorporating FPGAs in the networking protocols and utilizing other real-time human interface software allows for designing more sophisticated and more complex motor control schemes. Developing such interfaces increases the need for more FPGA on-chip resources or requires an additional microprocessor. Therefore, a system-on-chip approach allows more resources to be readily available for use by the design engineer in order to meet the timing and control complexity requirements.

In many power electronic applications, a flexible switching frequency is crucial to the design. Complementary switching in two or multi-level inverters, where each inverter leg has at least two IGBTs operating in a complementary fashion, requires precise dead-time allocation. An FPGA-based design allows for an accurate and

flexible programming of this dead-time for power devices with different switching frequencies. Moreover, the parallel computing nature of FPGAs provides high-precision gating signals to all switches in the design simultaneously and in a parallel fashion. Compensation methods have been proposed to mitigate the effect of dead-time on the design [42-44]. Another key aspect of using FPGAs is their capability for parallel computing as an accelerator where iterations or matrix multiplications could be 
pipelined to achieve a faster solution. As trigonometric functions are extensively used in power electronic applications, they could be implemented in hardware if there is a need for faster computation.

An FPGA is a good candidate for an application characterized by simple data objects and extensive parallelism. Contrary to floating-point operations on FPGAs [45] that demand more resources and have limited performance, a processor is a good candidate for managing design flow and floating-point calculations with readily available libraries. In this work, a novel design methodology utilizing both hardware and software resources is first explained. The remainder of this chapter is organized as follows. Section 5.2 presents a literature review and other relevant research work that have been carried out in the field of electrical motor drives. Section 5.3 provides a background on the functionality of a Zynq-based SoC. Section 5.4 explains the approach utilized in this chapter to develop this IM control application. Finally, the design evaluation and conclusion are given in Sections 5.5.

\subsection{Literature Review of Hardware/Software codesign}

A significant amount of research has highlighted the benefits of using FPGAs in a variety of application platforms such as automotive, industrial automation, aerospace applications, etc. Hardware/software codesign in power electronic applications has been presented in [46-48]. The authors in [46] have provided guidelines for SoC sensorless AC drive applications and presented an efficient hardware/software partitioning procedure. In their study, the final design was implemented on a soft-core processor (MicroBlaze) after some optimization criteria were met. Unlike our hardcore Zynq-based SoC, debugging soft-core designs is more difficult and is inefficient 
for real-time applications.

The authors in [47] used the Vivado High Level Synthesis Tool from Xilinx to implement a full-state feedback controller for a buck converter in an FPGA. A highlevel programming language is used to facilitate the design and simulation process of the controllers, where High-Level-Synthesis (HLS) [49] plays a vital role in converting a high-level code into a synthesized design compatible with FPGAs.

In reference [48], the authors have presented an implementation of Field-Oriented Control (FOC) of an AC motor, where they estimate the position of the rotor using an Extended Kalman Filter (EKF). In their design they used MATLAB as a third-party compiler solution to generate part of their code, a domain specific processor designed for automotive applications, an FPGA, and an interface circuitry between the FPGA and the processor.

Motor control requires precise calculations when estimating speed in sensorless motor control, implementing PI controllers, and estimating voltage magnitude. These values are used to create the gating signals for an inverter to apply proper voltages to the motor. A combination of a software and a hardware solution increases the flexibility and accuracy of the design.

In this work, HLS or any other third-party compiler were not used to create the code. The existing interface between the FPGA and the processor in a Zynq-based SoC is utilized to develop our design and implement a control strategy for an induction machine as a proof of concept. 


\subsection{The Zynq All Programmable SoC}

With advances in system-on-chip (SoC) devices, a combination of control, communication, and user interface with different processing bandwidths can be implemented on a single chip. The Zynq All Programmable SoC [50] integrates a high-performance Processing System (PS) with Programmable Logic (PL). The processing side of the Zynq consists of a dual-core ARM Cortex-A9 processor, a NEON coprocessor, and floating-point extensions that accelerate software execution. The hardcore processor can be used without the need to configure the PL. This means that the software code and the hardware interface placed on the FPGA fabric can be designed in parallel.

Applications can be partitioned into hardware and software tasks. The processing system that addresses tasks such as system management and control, math functions, and the user interface is well-suited for software implementation. The partitioning process in hardware/software co-design is an important part in the design of such systems and their applications, since it can lead to significant gains in system performance. The process of hardware/software partitioning involves deciding which system components are suited for implementation in hardware and which should be implemented in software. The Zynq All Programmable SoC allows the user to implement a complete system partitioned into software-based and hardware-based tasks that can be implemented in the PS and the PL, respectively. In most applications, the tasks in the software and hardware sides need to communicate with each other via a communication protocol suitable to linking these two sides. In Zynq-based system applications, the AXI interface is used as a communication bridge between the PS and PL. The AXI interface [51] is an open standard on-chip interconnect protocol that belongs to the ARM AMBA family of microcontroller buses. AXI protocol is 
optimized for FPGA implementation through a coordinated development with Xilinx. There are three types of AXI interfaces: AXI4, AXI4-Lite, and AXI4-Stream that are suitable for high-performance applications and data stream communication. AXI4 is basically used for high-performance memory-mapped requirements. On the other hand, AXI4-Lite is used for simple low-throughput memory-mapped communication, for instance, communication to and from control and status registers. AXI4-Stream is mainly used for high speed streaming data such as video streams. In this work, the AXI4-Lite was selected to be the preferred protocol for a communication environment between the processor core and the programmable logic.

\subsection{Algorithm Codesign Development}

A Variable Frequency Drive (VFD) is commonly used for controlling the operating shaft speed of an induction motor. There are different control schemes used in VFDs. For instance, Field-Oriented Control (FOC), Direct-Torque Control (DTC), and constant Volts/Hertz (V/f) are popular control strategies. These strategies, depending upon the application, could require more calculations, such as estimating speed in sensorless VFDs. In our design, the constant $\mathrm{V} / \mathrm{f}$ ratio is adopted as our control strategy. This method is suitable at or below the rated frequency of the motor. In order to keep the magnetic flux at its rated value, the voltage to frequency ratio must be kept constant. This simple control strategy is still widely adopted in many inexpensive VFDs today. It allows the motor to develop rated torque except at very low frequencies, where additional voltage boosting may be needed. The desired voltage and frequency values can easily be calculated on the PS side of the design and sent to the PL side. 
Figure 5.1 demonstrates the overall architecture used to develop this open-loop motor control application.

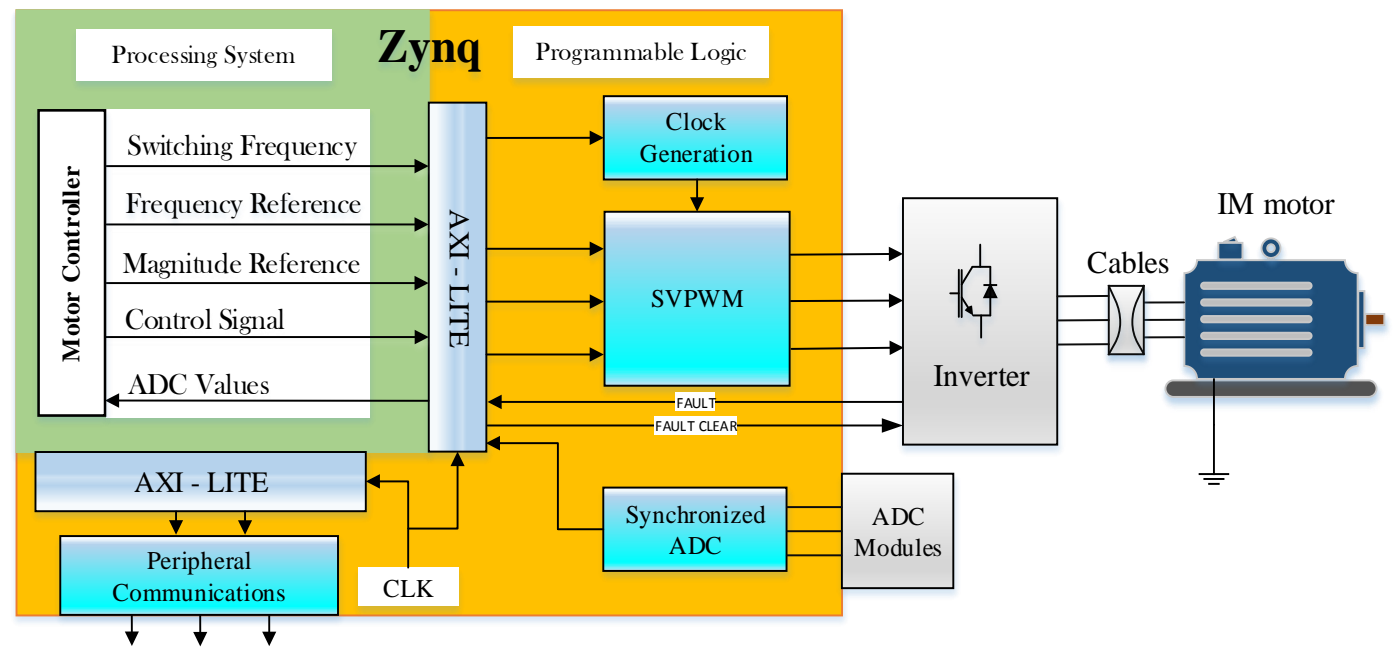

Figure 5.1: Power electronic components and control architecture of the design

In this section, the functionality of each register and block are explained in more detail. As mentioned earlier, a memory-mapped bus connects the PL and PS side registers. The clock cycle, (CLK), is set to $100 \mathrm{MHz}$ in this design. The AXI-LITE interconnection uses about 40 clock cycles to pass the data to the PL side.

\subsubsection{Hardware}

The PL side is responsible for three main blocks in this design: the Clock Generating, the Space-Vector Pulse Width Modulation (SVPWM), and the Synchronized ADC values. The Clock Generation block supplies a variable clock rate. This clock speed gets interpreted in the SVPWM block to generate a specific switching frequency range from $1 \mathrm{kHz}$ to $20 \mathrm{kHz}$. This rate can easily be updated from the PS side by changing the Switching Frequency register. The SVPWM calculation has been 
discussed in Chapter 2. The Verilog code of the Finite-State Machine module of the SVPWM has been added in Appendix B. An efficient implementation of the SVPWM on FPGA is also thoroughly discussed in [52]. The SVPWM is a well-known technique for computationally generating PWM signals. The fundamental frequency and magnitude of the sinusoidal PWM generated by this block is configurable by the Frequency Reference and Magnitude Reference registers in the PS side. These values are computed by a control strategy to adjust the speed and electromagnetic torque of the motor. The synchronized ADC block is able to convert the multiple analog signals to digital form with a constant sampling rate which is configurable by the designer. The gating PWM signals and sampled signals by the ADCs are kept at a fixed rate and the designers develop their controllers based on the sampling rate or the switching frequency. The PL side of the SoC allows this rate to be fixed and any complexity or delay for function execution in software does not have any effect on the sampling frequency of the ADCs or the SVPWM gating signals.

\subsubsection{Software}

The main control scheme is developed in the PS side of the design. Five registers are shared with the PL side through an AXI-LITE interconnect bus: Switching Frequency, Frequency Reference, Magnitude Reference, Control Signal, and ADC Values are updated based on the user design. The precise values of Frequency Reference and Magnitude Reference are calculated and placed in the registers. These register values determine the frequency and voltage magnitude being applied to the motor. The Control Signal register is used to manage the design flow, such as faulty situations, start and stop the operation, etc. This register has 32 bits and each bit has been allocated to a task such as start SVPWM, stop SVPWM, clear fault, and start ADC, 
etc.

Algorithm 1 describes the overall functionality of the whole PS side design. The operation starts by initializing Monitoring Devices such as LCDs, Switching Frequency modules, and ADC modules. The whole operation continues until a fault has been detected.

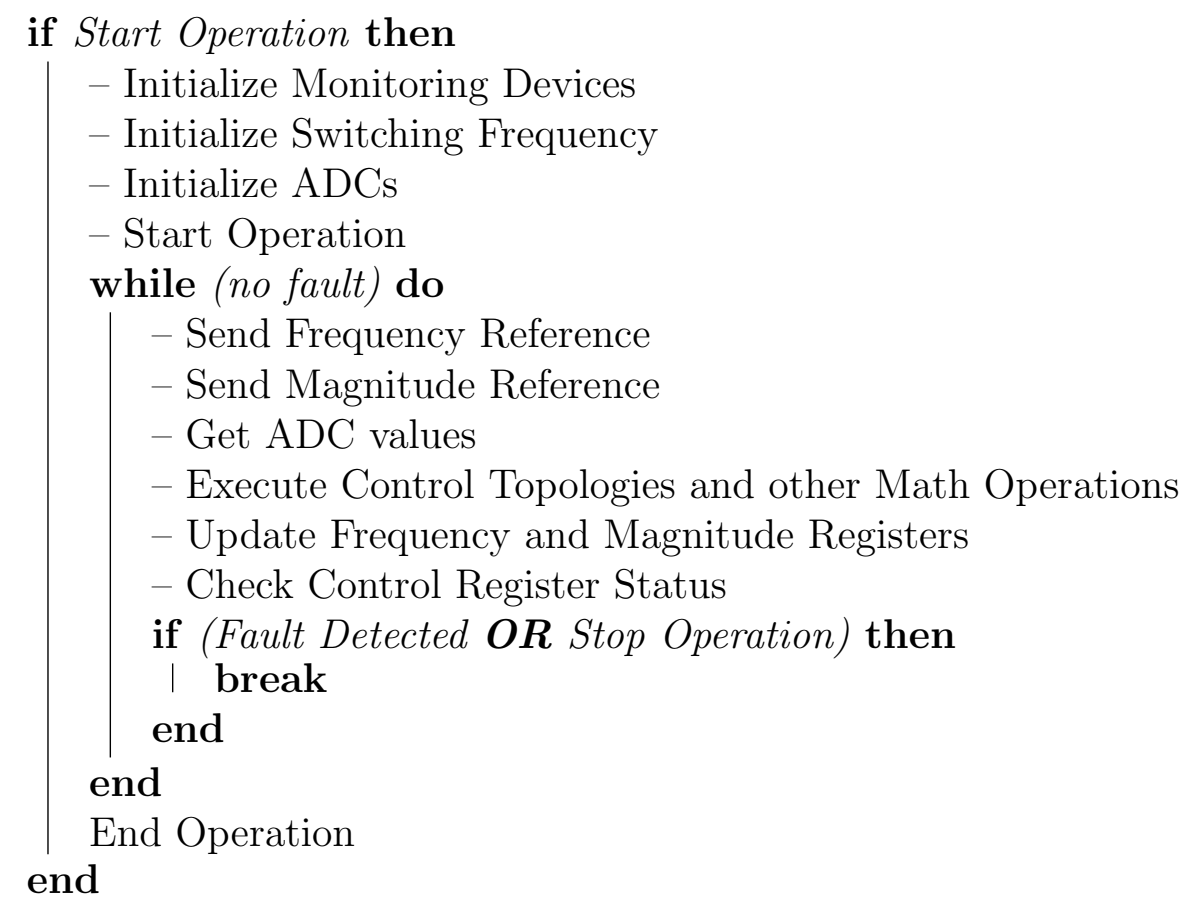

Algorithm 1: Design Solution for the PS side

\subsection{Design Evaluation}

In the previous sections, the differences between the PL and the PS side were explained. Several simulations have been done in order to evaluate the functionality of the PL side of the design. Also, both the PL and the PS designs of the motor control were tested to verify the complete design. The experimental setup and results are explained in this section. 


\subsubsection{Experimental Setup}

Experiments were conducted using a Xilinx Zynq-7000 SoC, a Zedboard, and a XC7Z020 and the manual of the board can be found in [53]. The board fabric runs on a clock speed of $100 \mathrm{MHz}$. The Xilinx EDA design suite, Vivado 15.4, was used for synthesizing and implementing the design. The Xilinx Software Development Kit 15.4 was used to initialize and evaluate the design. All of the PL side blocks are developed in Verilog. These blocks were then imported to an EDA design suite as an Intellectual Property (IP).

The Xilinx development board outputs the gating signals for a two-level inverter which supplies the required voltages and currents to a $130-\mathrm{W}, 30-\mathrm{V}$ three-phase induction motor. An intermediate circuit board is used to connect the FPGA board to the inverter. Table 5.1 provides more information about the parameters and rated values of the experimental equipment. Once a fault has been detected, the operation is halted.

Table 5.1: Hardware platform setup parameter values

\begin{tabular}{c|c||c|c}
\hline \hline Parameters & Values & Parameters & Values \\
\hline \hline Rated Power & 130 Watts & switching frequency & $20 \mathrm{kHz}$ \\
\hline Rated AC Voltage & 30 VAC & DC-Link Voltage & $42 \mathrm{~V}$ \\
\hline Number of poles & 4 & Motor Type & Induction Motor \\
\hline
\end{tabular}

\subsubsection{Experimental Results}

Three transient states are introduced from the PS side, as shown in Fig 5.2. The Frequency Reference register was initially set to $20 \mathrm{~Hz}$, at $t=0.2 \mathrm{~s}$ and then increased to $40 \mathrm{~Hz}$ and to $60 \mathrm{~Hz}$ at $t=0.51 \mathrm{~s}$. The measured motor current frequency is well matched to the Frequency Reference register value provided by the PS side. 

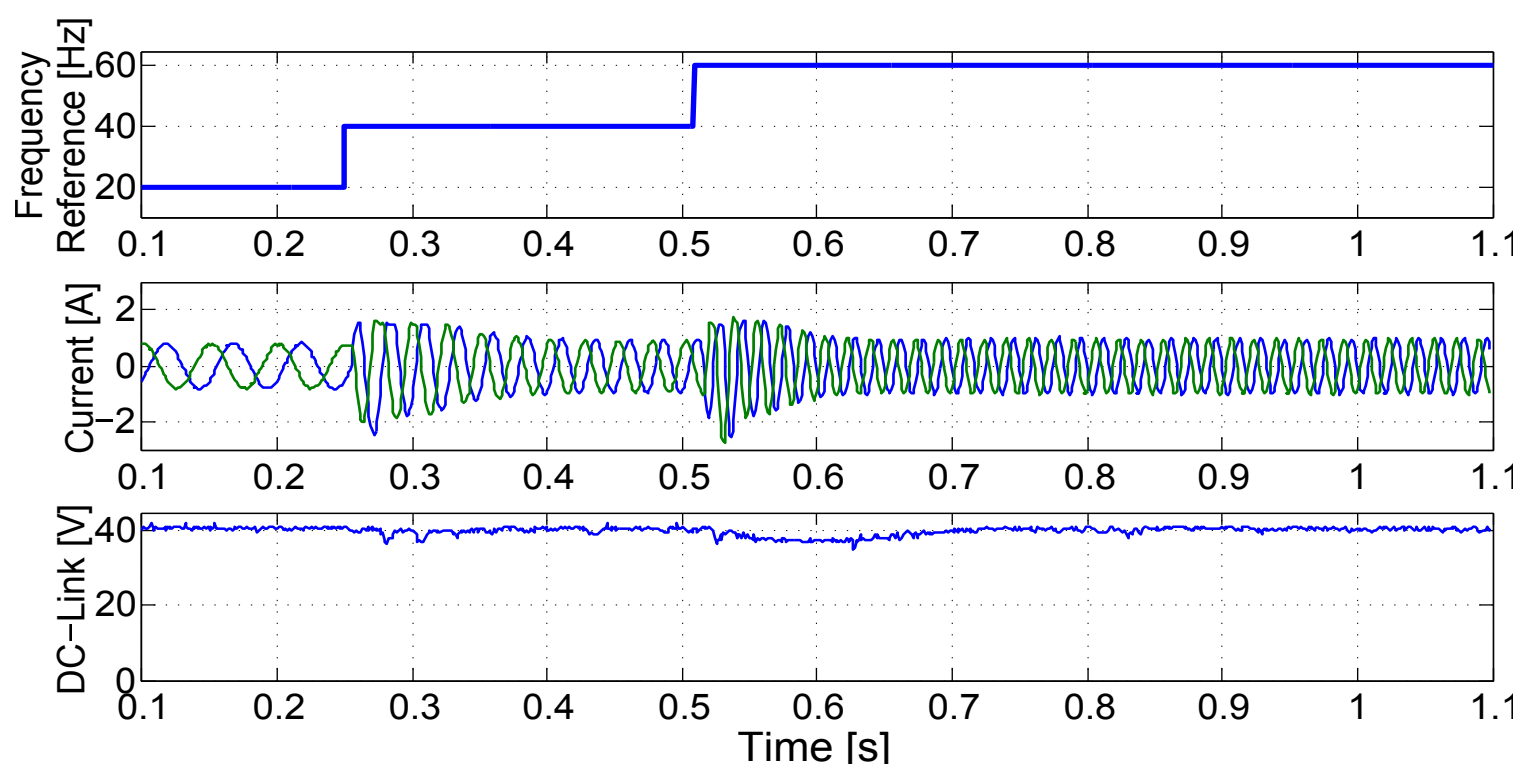

Figure 5.2: Experimental results from top to bottom: operating frequency command, current phase $a$ and $b$ of the motor, voltage of the DC-Link

However, due to its control method and the open-loop nature of the control design, the phase currents perform poorly during this transient condition. Figure 5.2 shows the measured currents in phases $a$ and $b$ as well as the DC-link voltage. It also shows the DC-Link voltage, which is fairly constant except for a voltage drop during a transient condition. This voltage drop could be reduced by a high performance motor control strategy or other methods that are outside the scope of this work. 


\section{CHAPTER 6}

\section{EXPERIMENTAL SETUP}

In this chapter, the experimental setup used to carry out the experiments is presented and a brief explanation of the functionality is provided. Throughout these experiments, some equipment were purchased and some were designed in the Power Lab at Boise State University. The equipment used for the experiments include two electrical motors, a three-phase inverter for supplying voltages and currents to the motors, a computational unit for calculation, and equipment for logging the real-time data. These equipment are discussed in detail in the following sections.

\subsection{Electrical Motors}

In these experiments, two motors are coupled to each other by the shaft. The 130 Watt, four pole, three-phase AC motor is used to obtain the desired shaft speed and the 250 Watt, 42 Volt DC motor is used to apply a load torque to the shaft. The three-phase inputs of the AC motor are connected to the three-phase inverter. The DC motor can be controlled for applying mechanical load and requires a 42 to 48 VDC lab power supply.

The motors were designed by MOTORSOLVER. The setup, the two motors coupled to each other, is sold under University Dyno-Kit products. These motors are fitted with encoders for speed measurements, as shown in Fig. 6.1. Also, a torque 


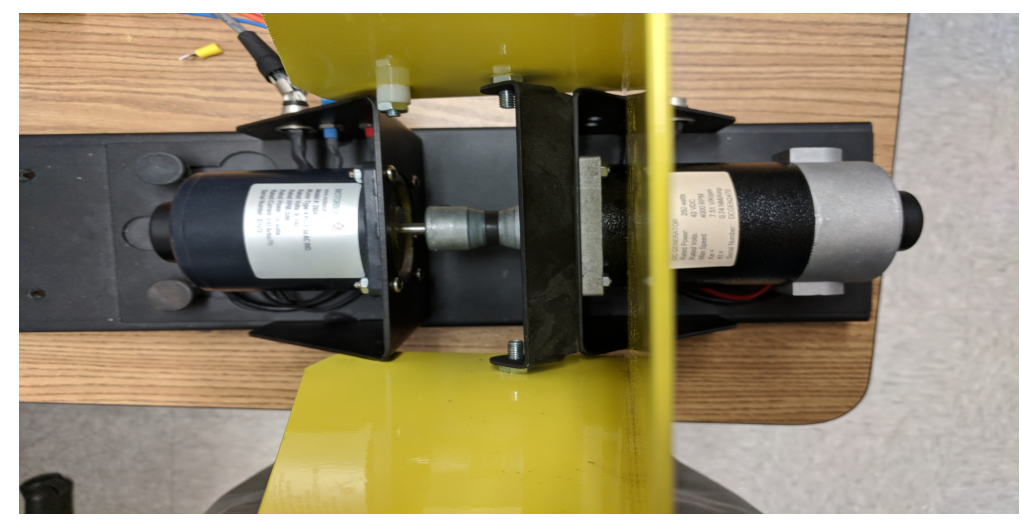

Figure 6.1: AC Motor and DC Motor coupled to each other

Table 6.1: Motor specifications

\begin{tabular}{rl||rl}
\hline & DC & AC \\
\hline \hline Rated Power & 250 Watt & Type & 4-pole, 3-phase \\
Rated Power & $42 \mathrm{VDC}$ & Volt & 30 VAC \\
Max. Speed & $4000 \mathrm{RPM}$ & RPM & $3396 \mathrm{RPM}$ \\
$k_{e}$ & $7.51 \mathrm{~V} / K_{r p m}$ & Rated Power & $130 \mathrm{Watt}$ \\
$k_{t}$ & $0.74 \mathrm{NM} / \mathrm{AMP}$ & Rated Current & 3.93 Ampere \\
\hline \hline
\end{tabular}

transducer can be fitted onto the shaft. The specifications of the electrical motors are given in Table 6.1.

\subsection{Three-Phase Inverter}

A medium voltage three-phase inverter is required to carry out the experiment. This three-phase inverter is connected to the Computational Unit and to the AC electrical motor. The controller in the Computational Unit uses the appropriate PWM signals to control the speed of the motor by applying the proper voltages from the three-phase inverter. This inverter has six isolated and independent MOSFETs. Each of the switches can be controlled independently. This board was designed in the Power Lab at Boise State University as a two-level, $100 \mathrm{~V}$, three-phase inverter with a 
maximum current output of 21 Arms. The main feature of this board, compared to the commercially-available inverters in the Power Lab is that all of its switches can be controlled independently and there are some specific capacitor size consideration for the gate drivers. This feature allows turning off one of the switches at any given time in order to evaluate the developed detection algorithm, whereas in the commercial three-phase inverter each of the switch pairs are operated in a simultaneous fashion.

\subsubsection{Board Design Specifications}

This board was designed to tolerate 500 VDC at the DC-link and currents around 21 Arms. The circuit was designed by using the EAGLE program. The developed prototype and the layout are shown in Fig. 6.2 and Fig. 6.3. This board consists of three main components: Sensing Units, a Low Voltage Unit, and a High Voltage Unit, as shown in Fig.6.2. These components are briefly discussed in the following subsections, respectively.

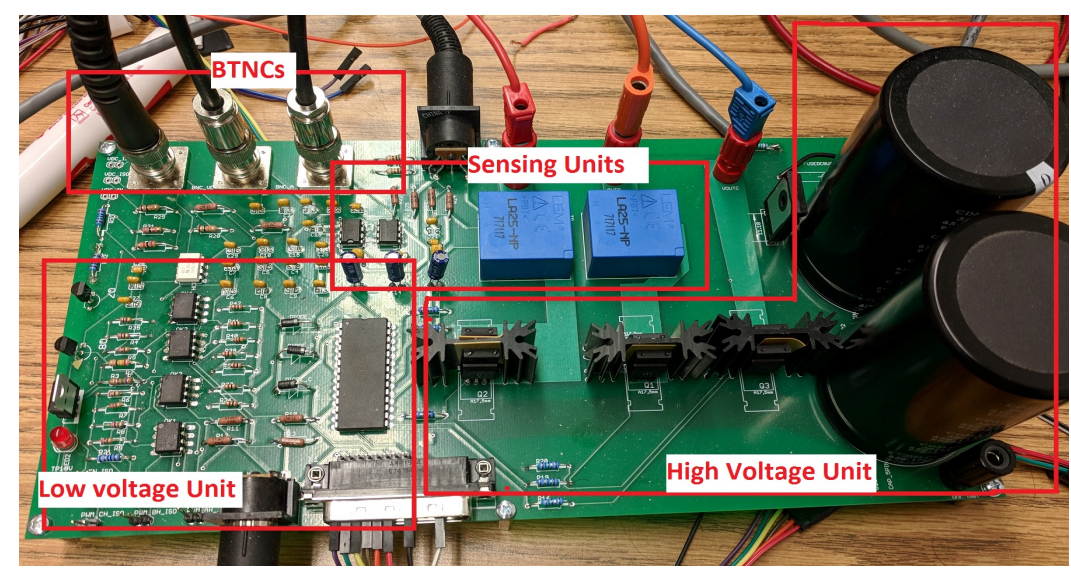

Figure 6.2: Printed circuit board of the three-phase inverter 


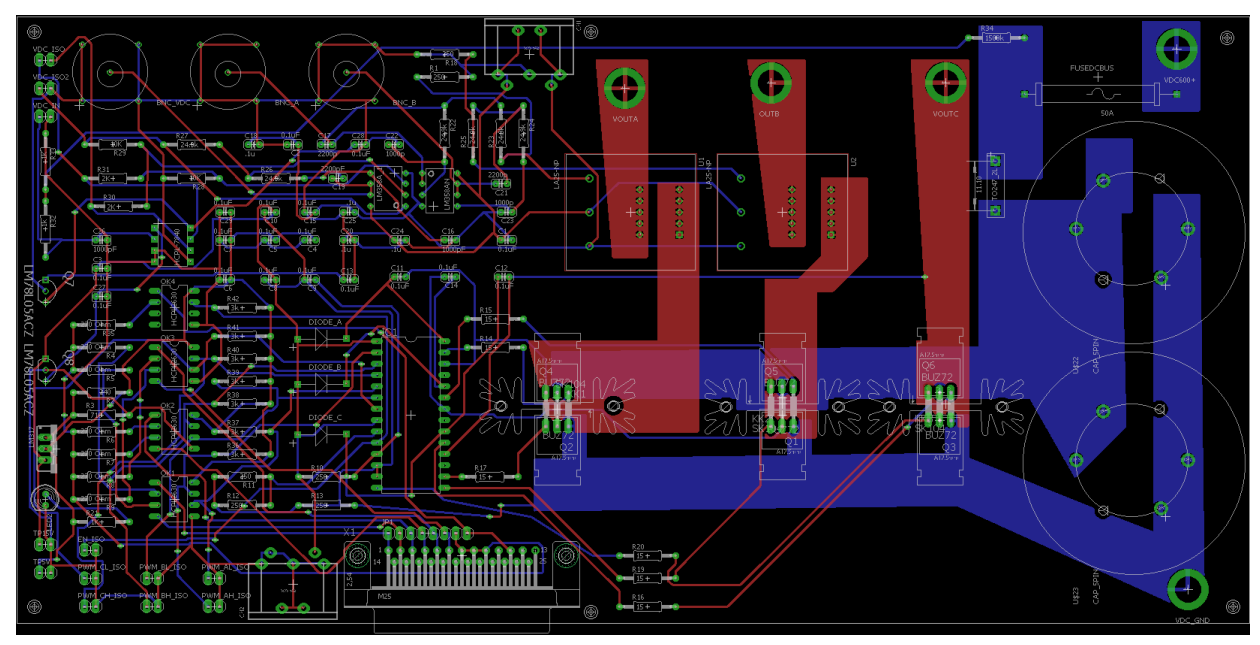

Figure 6.3: Layout of printed circuit board of the three-phase inverter

\section{Sensing Units}

There are three signals that are sensed in this inverter. These signals are two of the phase currents (phase $a$ and phase $b$ ) and the DC-Link voltage.

Two of the current signals are measured using Hall-effect sensors (Part: LA 25-NP, LEM) as shown in Fig. 6.4. The voltage of the phase b is VSB1 and the current of the phase $b$ is routed through the Hall effect sensors. This sensor acts as a small current reducer and its ratio can be modified using its connections (pins 1 through 10).

More information on the input-output connection is available in the sensor's datasheet. The voltage across R18 is measured in order to be logged (CSB). The ratio between the current through the sensor and the measured voltage of R18 is 1.96. This means that one Ampere is equal to 0.5102 Volt. Therefore, a ratio of 1.96 is multiplied to the measured voltage in order to get the correct current readings.

A test for verifying the ratio was carried out. This test is done to verify the correctness of the ratio for a wide range of the current. The voltage obtained from 
the corresponding current under test is stored and shown in Fig. 6.5.

The DC-Link voltage is reduced using a optoisolator and an linear amplifier (HCPL-7840), as shown in Fig. 6.6. This circuit will isolate the DC-link and also reduce the voltage of the DC-link to an ADC voltage range $( \pm 10 \mathrm{~V})$.

These three signals are connected to the BNC outputs, as shown in Fig 6.2. The voltages across these BNCs are fed to the ADCs connected to the Computational Unit and the real-time data logging unit dSPACE.

To determine this value, a constant current of zero $\mathrm{A}$ is fed to the the sensor to get the bias value. A bias value of $-56 \mathrm{mV}$ for phase $a$ and a bias value of $-8 \mathrm{mV}$ for phase $b$ were obtained. These two values will be accounted for in the two-phase current measurements.

\section{Low-Voltage Unit}

In this design, the components connected to the 12 VDC and the 15 VDC are considered low voltage components such as the linear amplifier, isolated PWM gates, and gate-source signals of the switches. The $\pm 12 \mathrm{VDC}$ and $15 \mathrm{VDC}$ power supplies are connected to the board through DIN-5 connectors, as shown in Fig. 6.7.

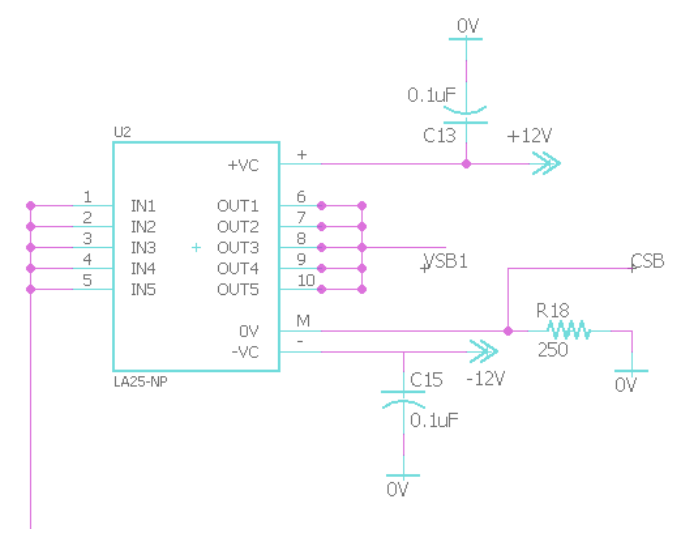

Figure 6.4: The circuitry of the sensor of the phase $b$ current 


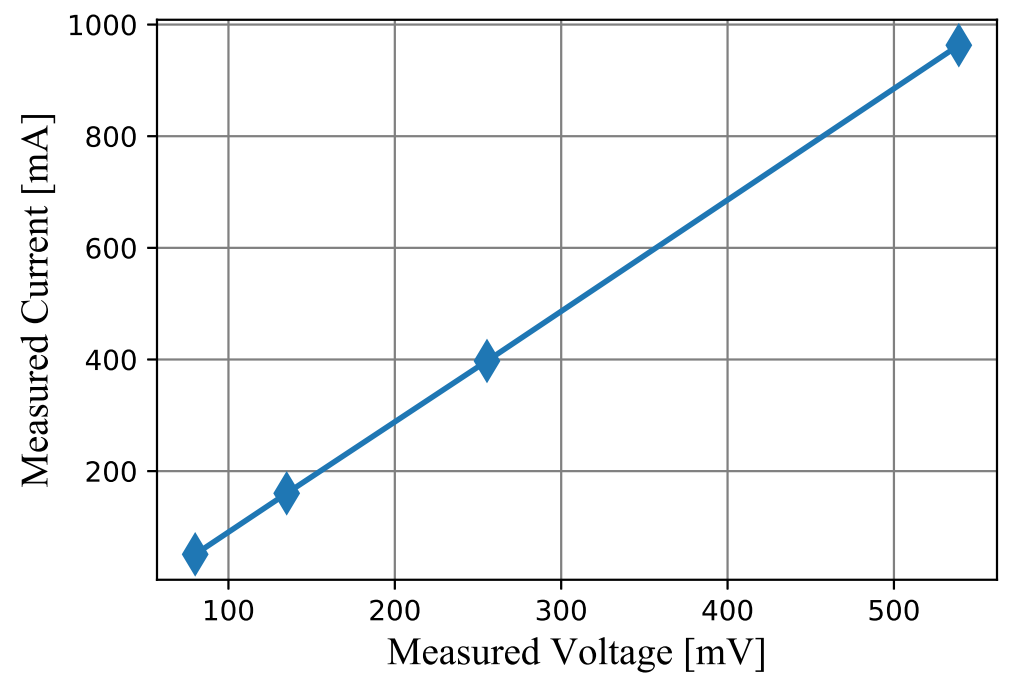

Figure 6.5: Measured voltage vs. applied current in the current sensor

\begin{tabular}{|c|c|c|}
\hline$P \$ 1$ & VDD1 & VDD2 \\
\hline $\mathrm{P} \$ 2$ & VIN+ & YOUTt \\
\hline$P \$ 3$ & VIN- & VOUT- \\
\hline$P \$ 4$ & GND1 & GND2 \\
\hline
\end{tabular}

Figure 6.6: The circuitry of the voltage sensing of the DC-Link

In this stage, 3.3 V PWM signals from an FPGA board are isolated and converted to $12-\mathrm{V}$ PWM signals. This isolation is done by the high-speed optocouplers (Part: HCPL2630). Figure 6.8 shows the D-SUB 25-pins connector where the PWM signals are connected to the board from an FPGA board. The JP1 component in Fig. 6.8 provides 8 pins for monitoring six PWM signals, a ground signal, and an Enable signal. The pins from 1 through 6 in the X1 component are the PWM inputs to the board and the notation $\mathrm{H}$ and $\mathrm{L}$ denotes the upper and lower switches of each phase, respectively. 

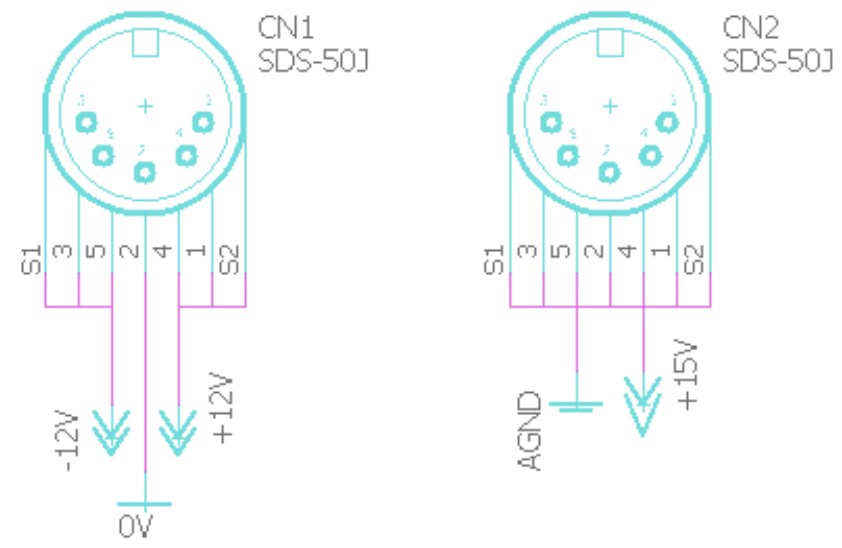

Figure 6.7: The 5 DIN connections of the two power supplies

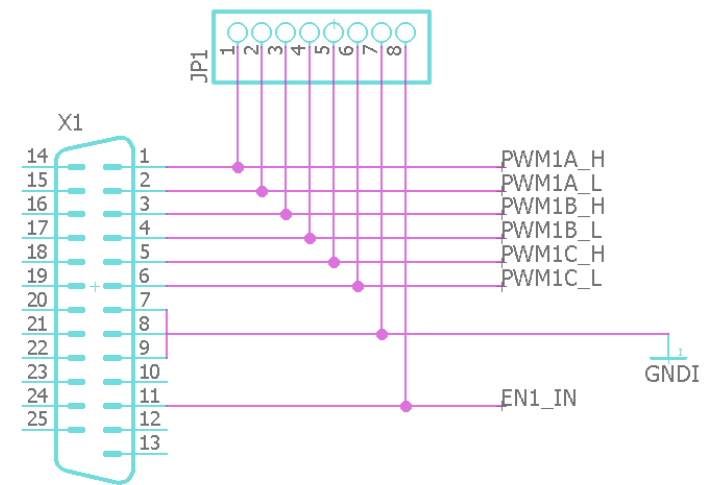

Figure 6.8: The circuitry of the PWM inputs

\section{High-Voltage Unit}

There are three main components in the High-Voltage Unit. They are the DC-link capacitors, power MOSFETs, and three-phase gate drivers. Each DC-link capacitor used in this board is $1000 \mu F$, 500 WDC (Part: series 382LX, CDM Cornell Dubilier) and the Power MOSFETs are N-channel 600V 37.9 A (Part: IPA60R099C6XKSA1, Infineon). The schematic of the gate driver for one leg is shown in Fig. 6.9.

Figure 6.9 shows the circuitry of a three-phase gate driver for one leg. In designing the gate driver, specifically for studying an open-switch fault, the size of $C 1$ (voltage 


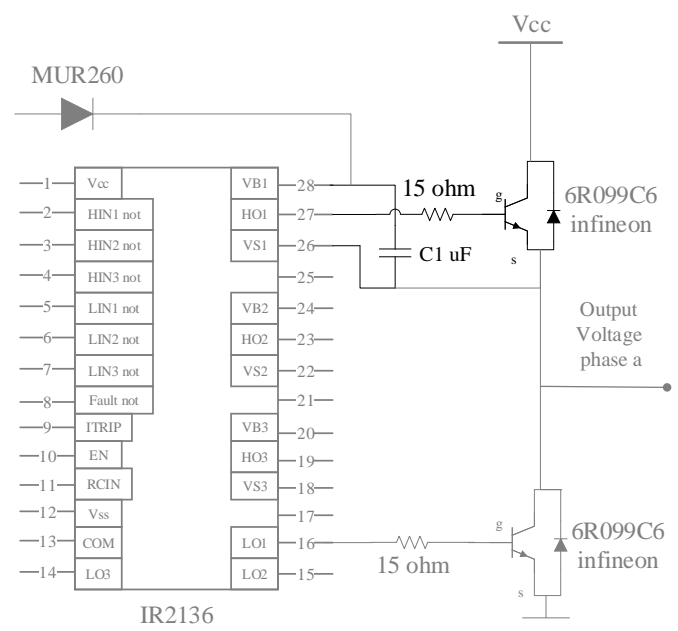

Figure 6.9: Three-phase gate driver and switches for one leg

between VB1 and VS1) plays an important role. The voltage across $C 1$ must be stable and constant for the duration of the fault. In this design, the capacitor size of $220 \mu F$ has been used. This value has shown enough stability to keep the voltage across $C 1$ stable and constant for a long time, about 10 seconds. Figure 6.10 shows the voltage across $C 1$, gate-source signals applied to the top switches, and three-phase current of phase $a$ during pre- and post-fault. A constant and stable voltage across the $C 1$ allows for creating scenarios for both short-circuited and open-switch faults.

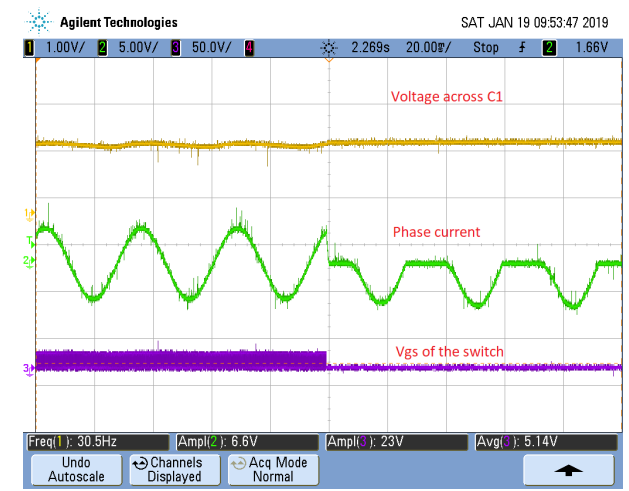

Figure 6.10: Three-Phase gate driver and switches for one leg 


\subsection{PWM Signals}

The functionality of the board has been tested and the results are presented in this section. Figure 6.11 depicts PWM signals for all of the top switches $S_{1}, S_{2}$, and $S_{3}$. At time $t=2.0775 \mathrm{~s}$, the algorithm applies the fault at the gate-source of the switch $S_{1}$, which keeps the signal at zero for a long time.

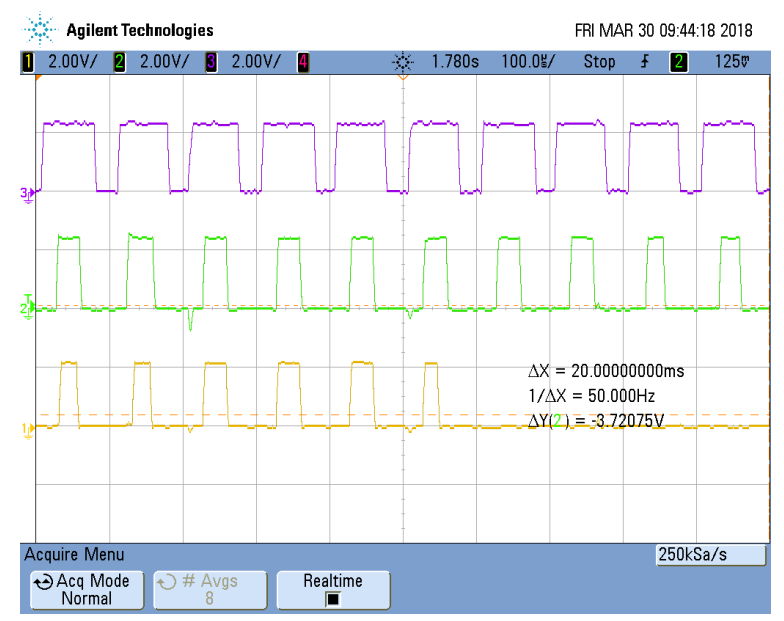

Figure 6.11: SVPWM signals for the three-phase inverter during pre- and post-fault

\subsection{Computational Unit}

A ZedBoard, evaluation board, has been used for the computational unit. The ZedBoard board is in the Xilinx's Zynq device family. This computational unit and the design platform have been thoroughly described in Chapter 5. Along with an FPGA board, a three-channel 16-bit high sampling frequency ADC (Santa Fe, Maxim) is also used. The system-on-chip architecture, explained in Chapter 5, allows part of the design such as the ADC driver and the PWM generation to be implemented on the FPGA board and the remainder of the design on the ARM microcontroller. 


\subsection{Logging Data}

The ControlDesk module made by dSPACE is used for data logging. The interface diagram is designed in SIMULINK (Real-Time Interface to SIMULINK RTI1104) and the $\mathrm{C}$ code is deployed to the real-time processor. The ControlDesk software in dSPACE is used to store and log all of the real-time data. Figure 6.12 depicts the user interface created for this project. The logged signals are the three-phase current, the speed of the motor, the DC-Link voltage, and the fault signals.

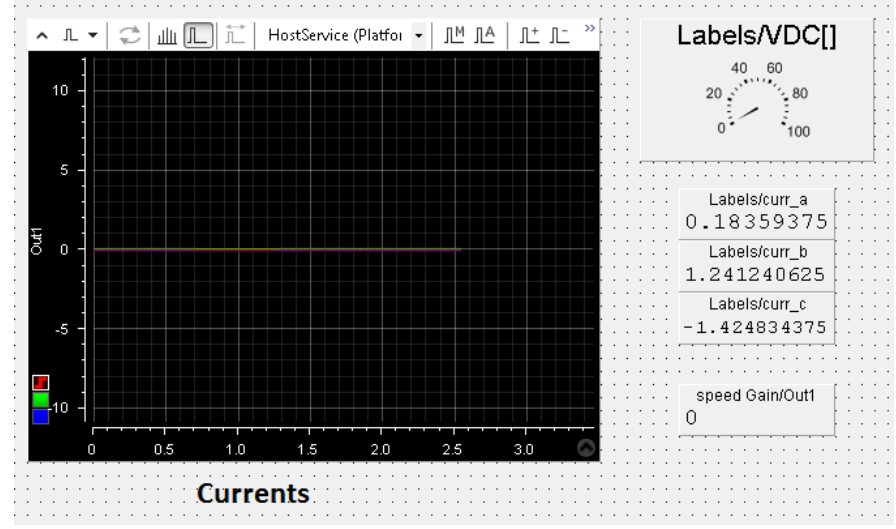

Figure 6.12: dSPACE ControlDesk diagram of user interface for real-time data logging

\subsection{Conclusion}

In this chapter, all of the setup and equipment used for the experiments were explained. These experiments have been carried out using the inverter designed by the Power Lab at the Boise State University. The details for designing a three-phase voltage-source inverter have also been discussed. 


\section{CHAPTER 7}

\section{CONCLUSION AND FUTURE WORK}

\subsection{Conclusion}

The proposed fault-tolerant topology is able to reconfigure the circuit so that the open-switch, the short-switch, the short-phase, and the open-phase faults can be mitigated. The common-mode voltage and current are minimum during pre-fault. Using this topology and the new switching strategy, the common-mode voltage and the common-mode current are reduced for open-switch and short-switch faults and are eliminated during post-fault operation of the inverter for open-phase and short-phase faults.

Furthermore, a new detection algorithm has been introduced and implemented. The functionality of this new method is verified by the experimental setup for various fault-angles and different operating speeds of an induction motor. All of these tests have been carried out using the three-phase voltage-source inverter made by the Power Lab at Boise State University. A new SoC platform has been introduced in this work, where the fault detection design and motor control were programmed and implemented. The performance of this new algorithm have been compared to another non-invasive method in the literature, where the proposed algorithm has a superior performance in terms of detection time. This method, as mentioned earlier, is a non-invasive method and can be implemented easily on the micro-controller of the 
fault observatory of the motor drive.

\subsection{Future Work}

There is possible work that can be performed as future research on each of the fault detection and the fault-tolerant topologies. For the topic of a fault-tolerant topology, the open-phase fault requires an induction motor with a capability of opening the phase or shorting any phase of the motor. The open-phase or the short-circuited phase fault can be tested once the required motor is available.

The fault detection method, absolute covariance, that has been proposed in this work, is for a three-phase induction motor and a five-phase PMSM which use a voltage-source inverter. The three-phase voltage source inverter has been built and the algorithm has been verified. This experimental verification of the five-phase or any multi-phase voltage-source inverters are proposed as a future work. 


\section{APPENDIX A}




\section{Parameters used for FOC}

Table A.1: Simulation parameter values

\begin{tabular}{c|c|l}
\hline \hline Module & parameters & values \\
\hline \hline machine & Sampling Time, $T_{s}$ & $10 \times 10^{-6}$ \\
\cline { 2 - 3 } & Nominal Power, $P_{n}$ & $10 \times 10^{-6}$ \\
\cline { 2 - 3 } & Rated Frequency, $f_{n}$ & $10 \times 10^{-6}$ \\
\cline { 2 - 3 } & Voltage, $V_{l l}$ & $10 \times 10^{-6}$ \\
\cline { 2 - 3 } & Inertia, $J$ & 3.1 \\
\cline { 2 - 3 } & Friction Factor, $F$ & 0.08 \\
\cline { 2 - 3 } & Stator Resistance, $R_{s}$ & $14.85 \times 10^{-3}$ \\
\cline { 2 - 3 } & Stator Inductance, $L_{\ell s}$ & $0.3027 \times 10^{-3}$ \\
\cline { 2 - 3 } & Rotor Resistance, $R_{r}$ & $9.295 \times 10^{-3}$ \\
\cline { 2 - 3 } & Rotor Inductance, $L_{\ell l}$ & $0.3027 \times 10^{-3}$ \\
\cline { 2 - 3 } & Pair Poles, $P$ & $29.295 \times 10^{-3}$ \\
\hline controller & $k_{i}$ for speed & 1900 \\
\cline { 2 - 3 } & $k_{i}$ for flux & 30 \\
\cline { 2 - 3 } & $k_{i}$ for voltage & 0.009 \\
\cline { 2 - 3 } & Torque Saturation & 1200 \\
\cline { 2 - 3 } & Voltage Saturation & $\sqrt{\frac{2}{3}}$ \\
\cline { 2 - 3 } & $k_{p}$ for speed & 300 \\
\cline { 2 - 3 } & $k_{p}$ for flux & 100 \\
\cline { 2 - 3 }$k_{p}$ for voltage & 0.08 \\
\cline { 2 - 3 } & Flux Saturation & 2 \\
\cline { 2 - 3 } & DC-Link Voltage, $V_{D C}$ & $460 \mathrm{~V}$ \\
\hline
\end{tabular}


APPENDIX B 


\section{Verilog Code for SVPWM}

The following snippet presents the finite-state machine used in Verilog to create SVPWM gate signals with deadtime. The simulation of the overall design is shown in Fig. B.1.

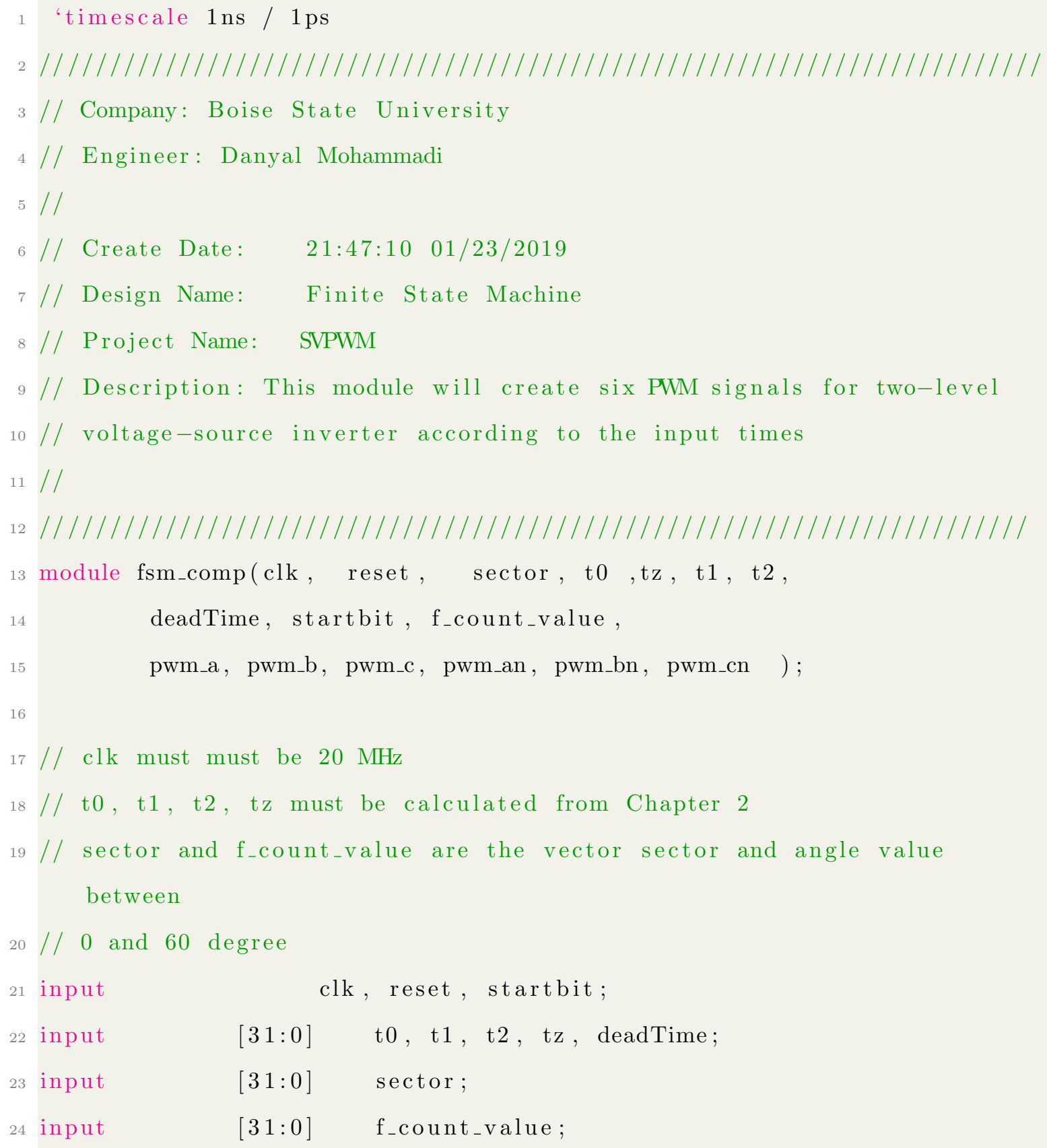




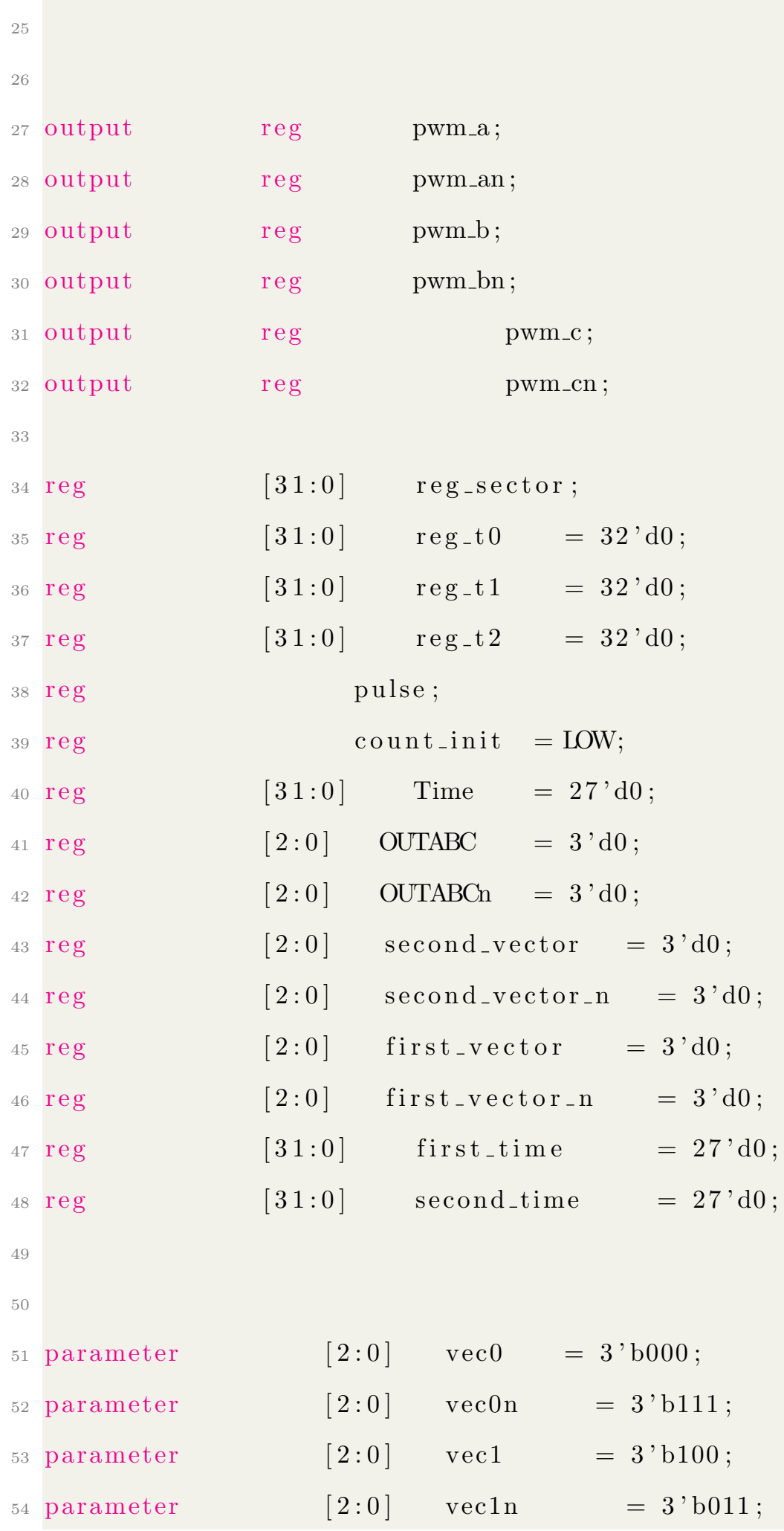




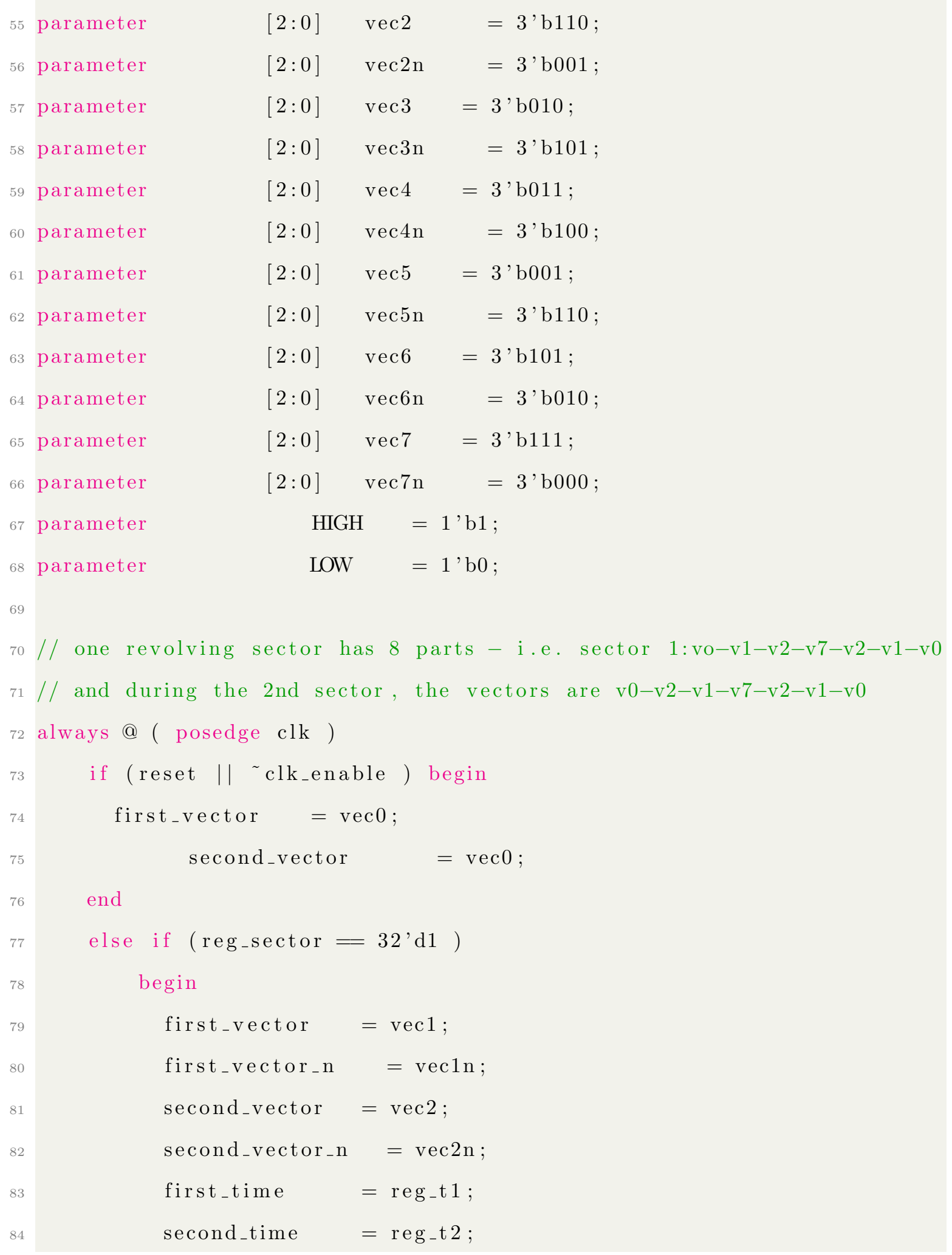




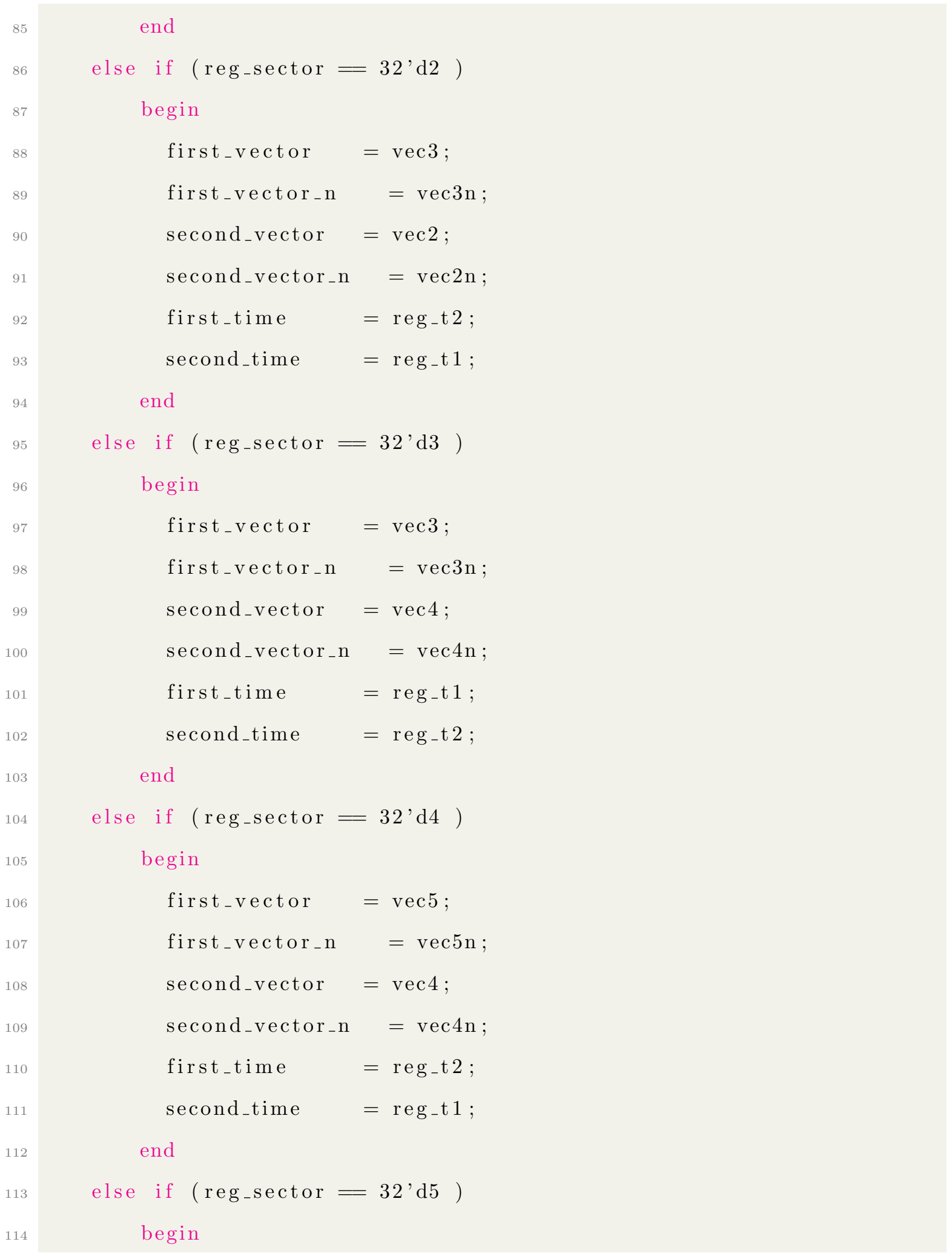




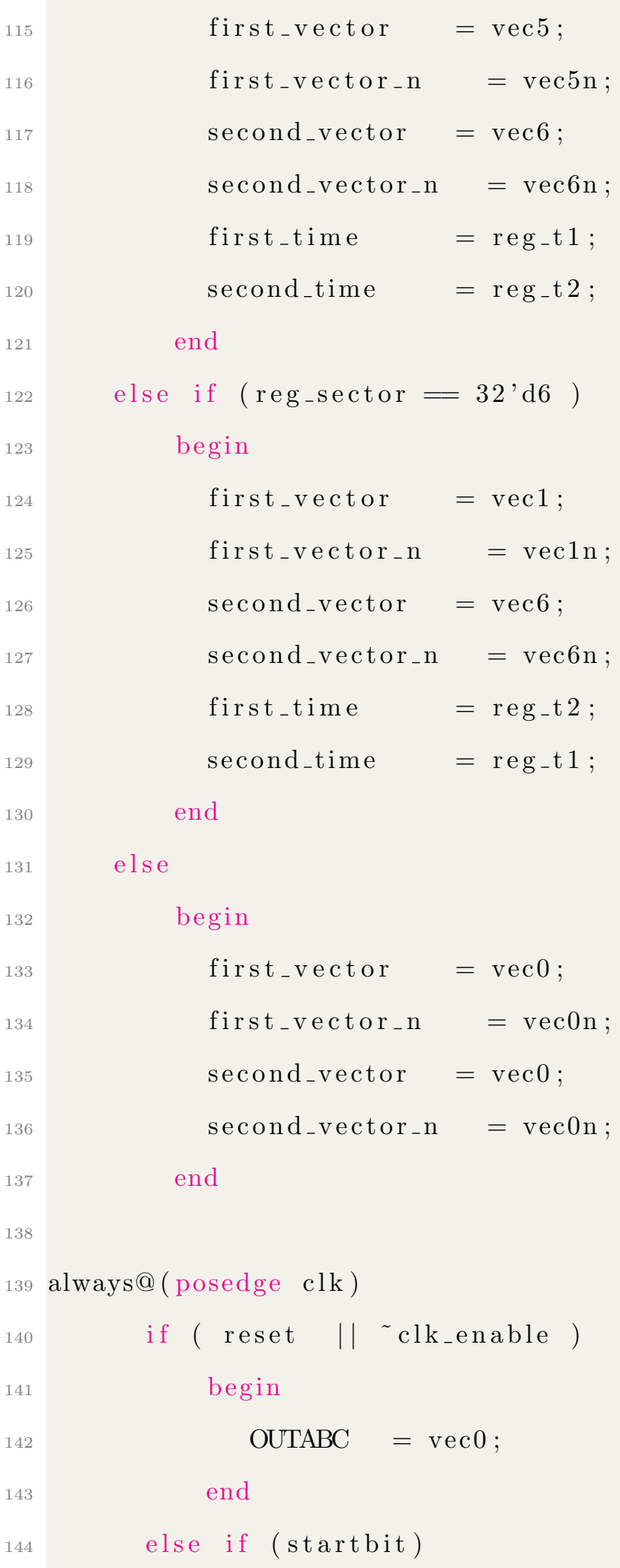




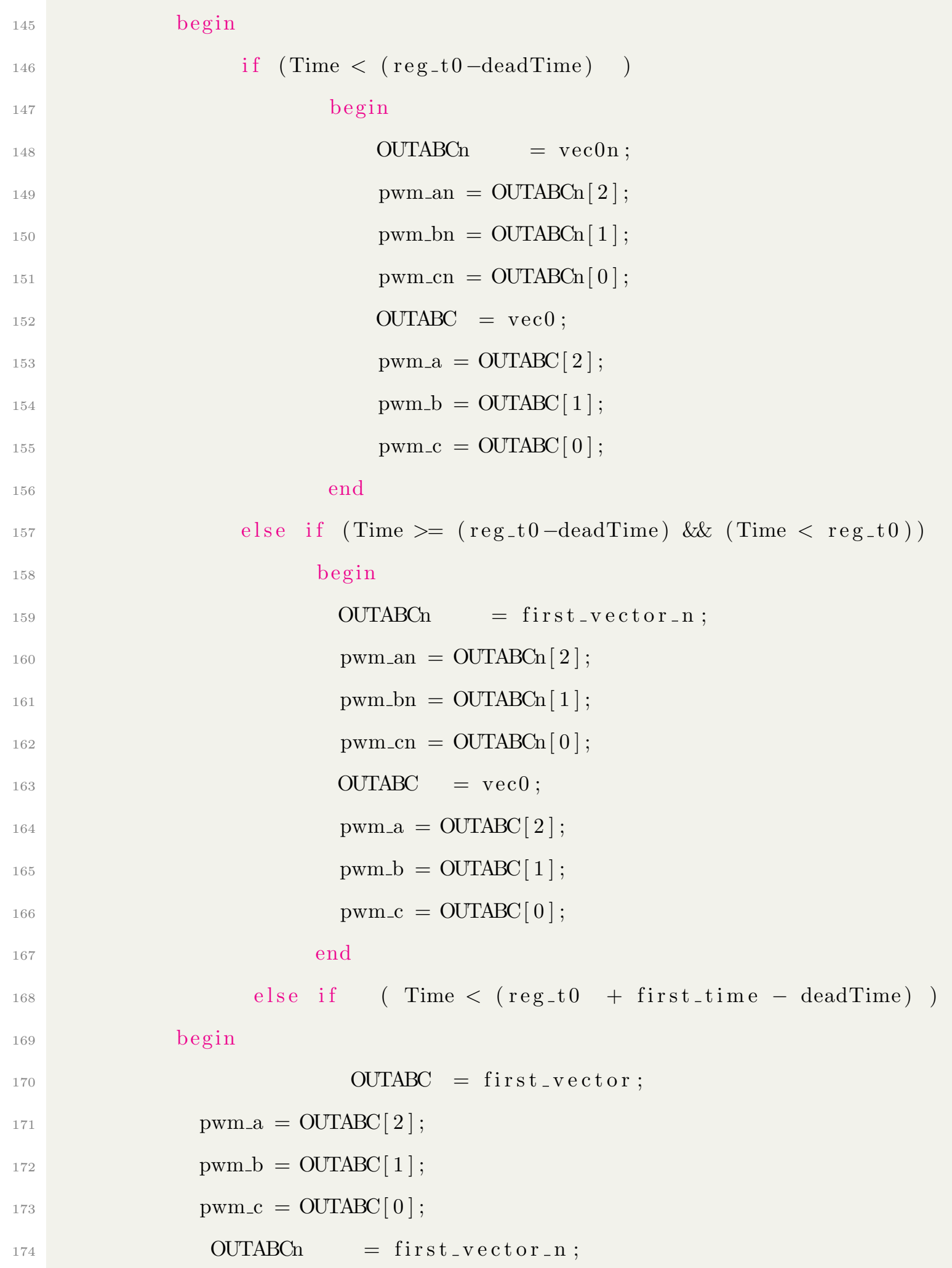




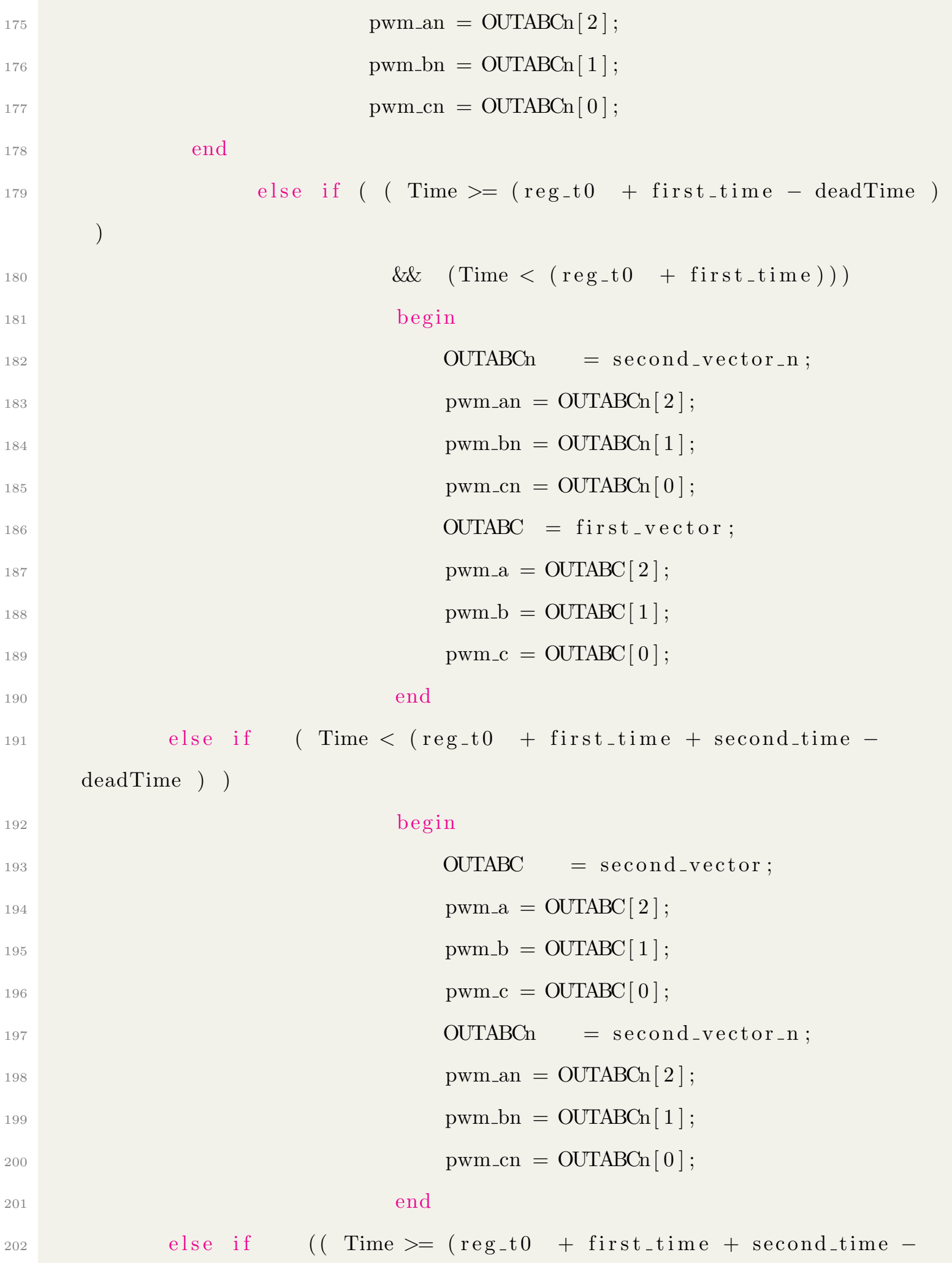




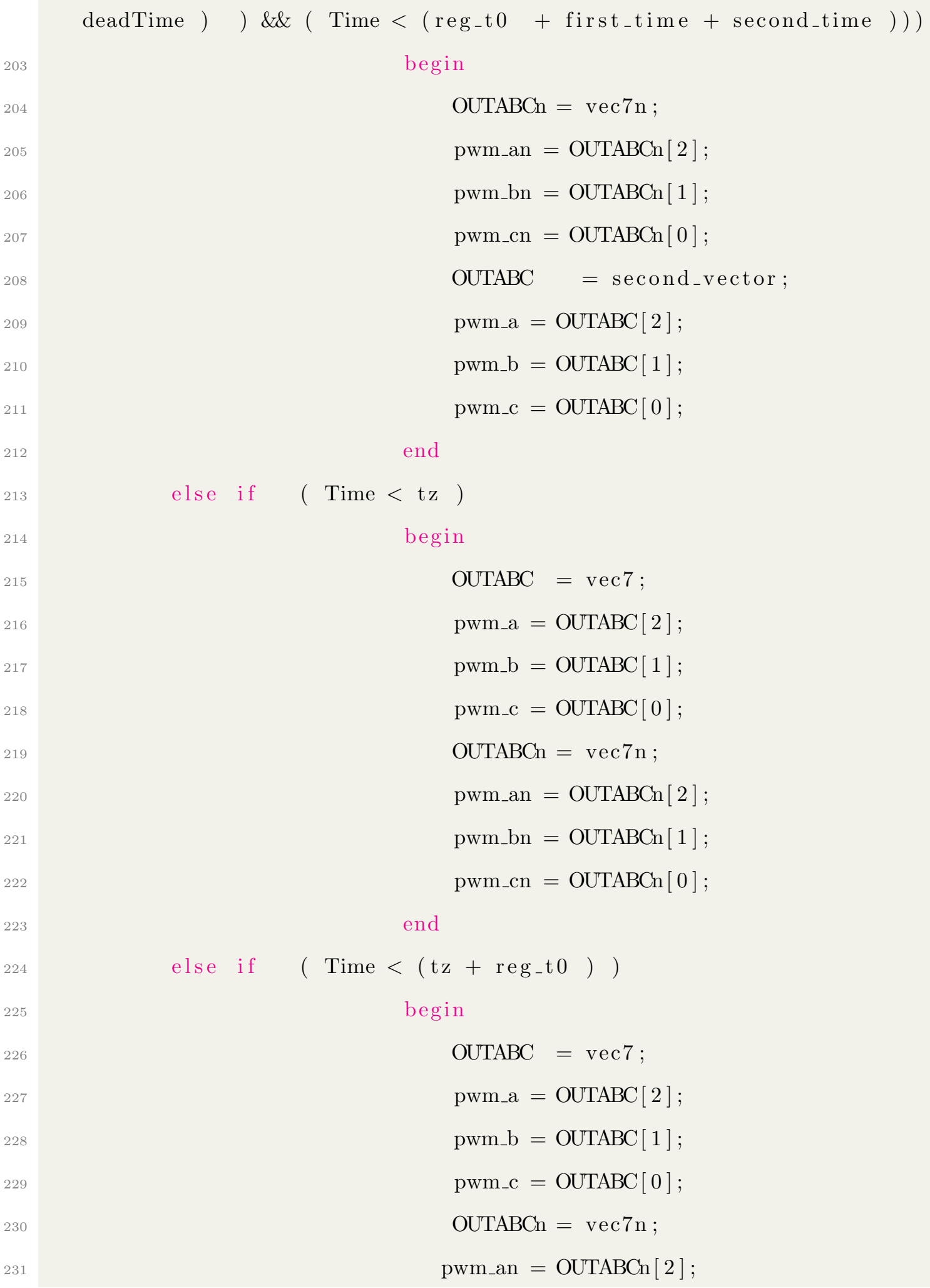




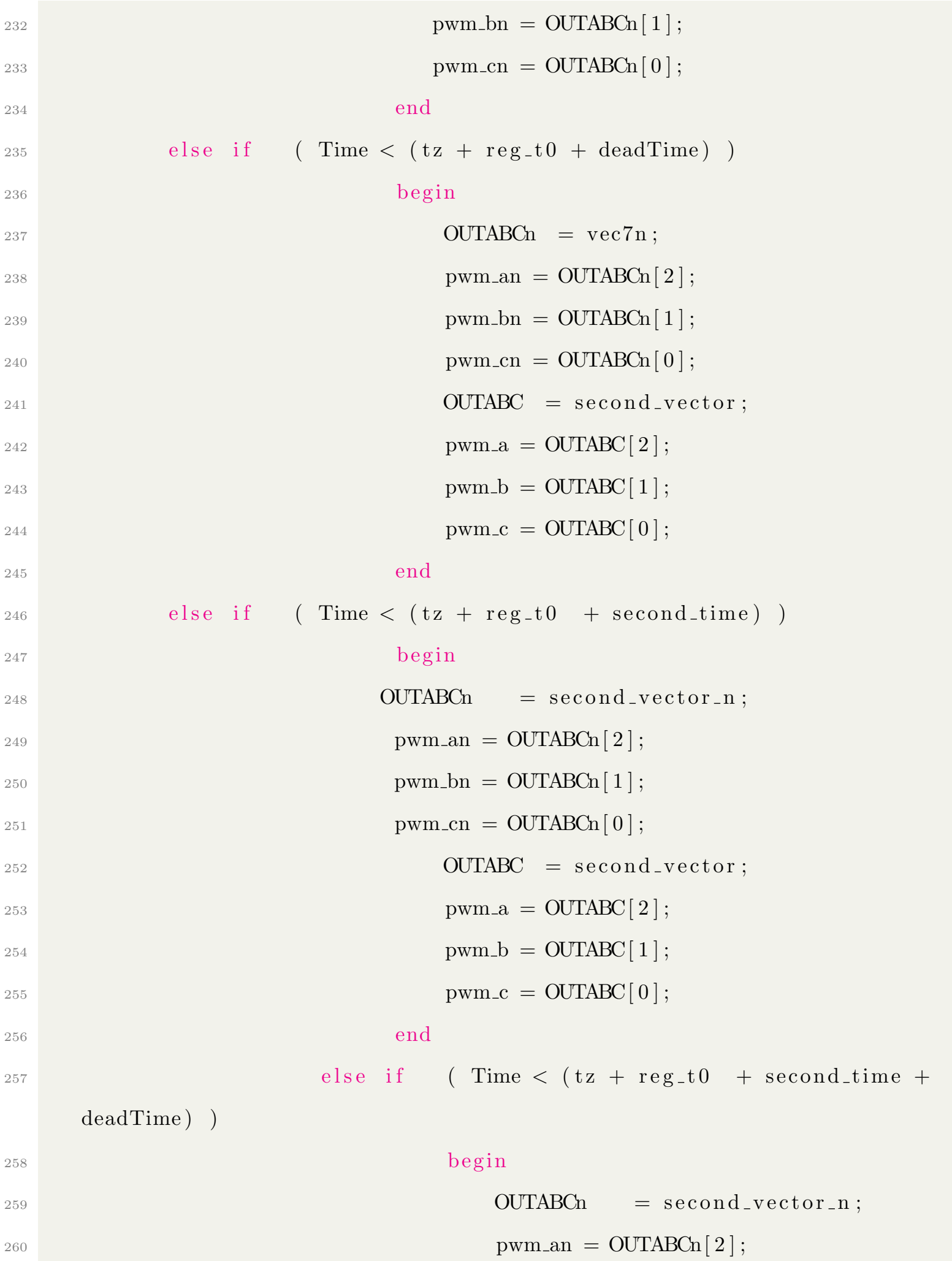




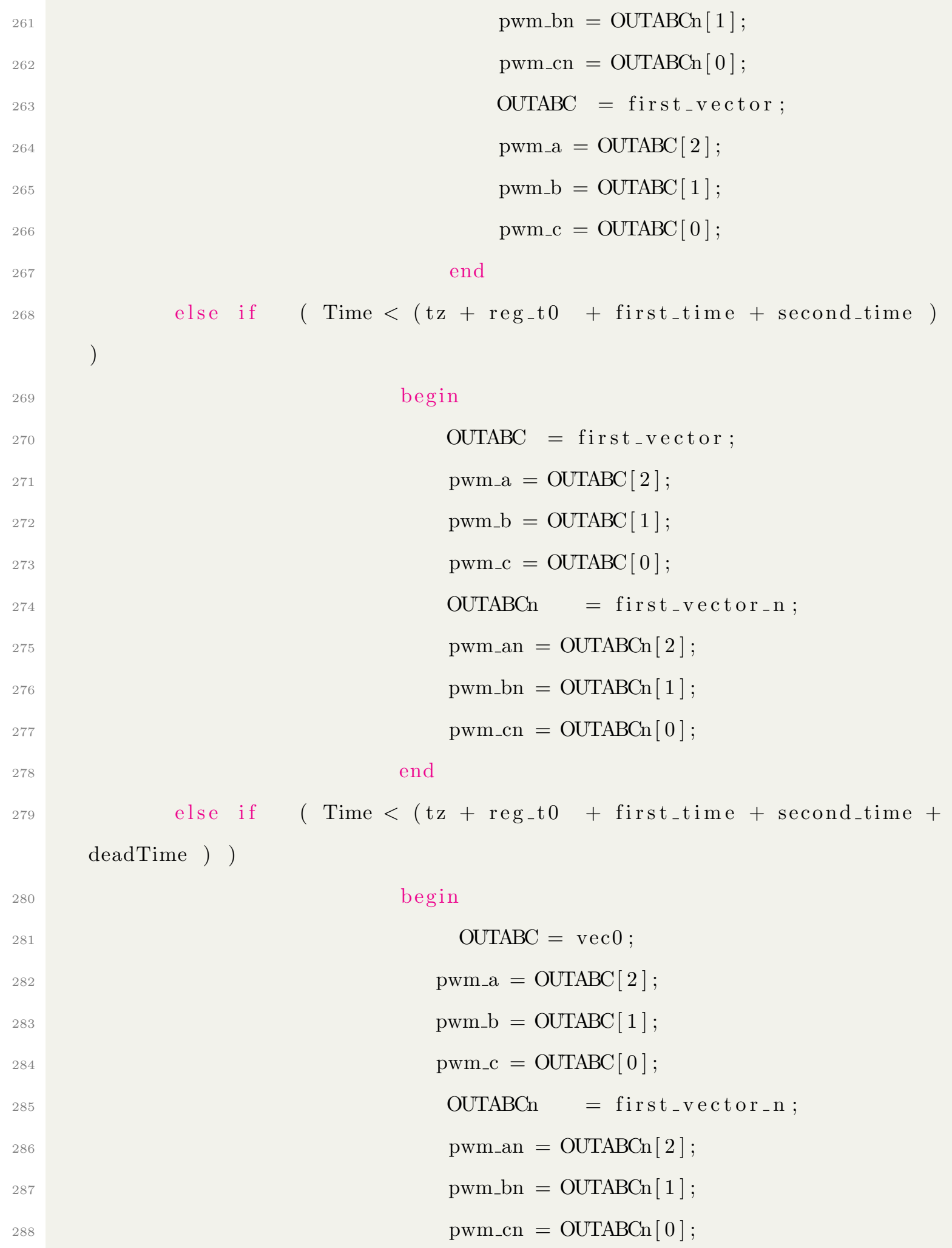




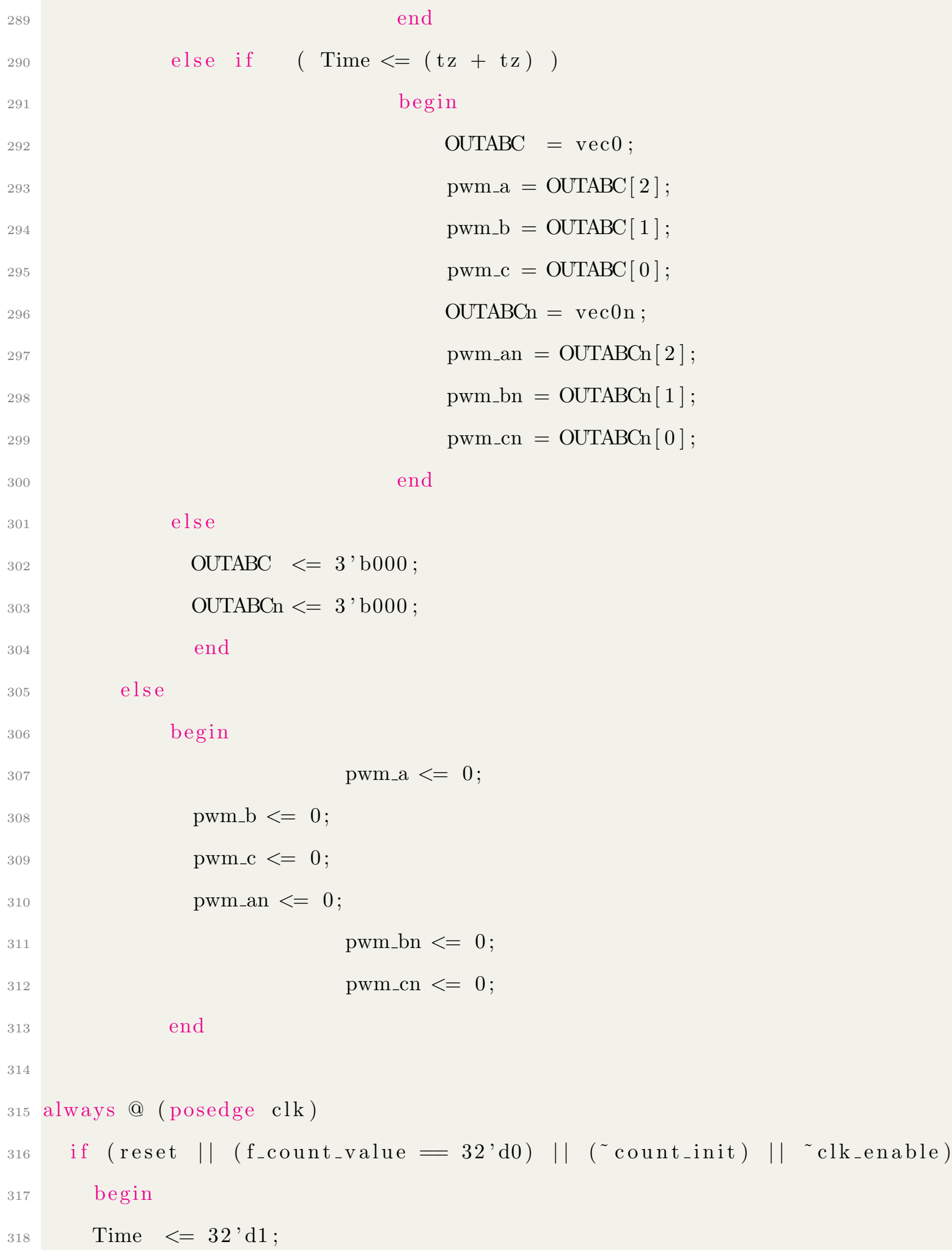

317

318

end

$$
\text { else if } \quad(\text { Time }<=(t z+t z))
$$

begin

OUTABC = vec0;

pwm_a $=$ OUTABC $[2]$;

pwm_b = OUTABC $[1]$;

$\operatorname{pwm}_{-} \mathrm{c}=\operatorname{OUTABC}[0]$;

OUTABCh $=\operatorname{vec} 0 \mathrm{n} ;$

pwm_an = OUTABCn $[2]$;

pwm_bn $=$ OUTABCn [1];

pwm_cn $=$ OUTABCn $[0]$;

end

else

OUTABC $<=3$ 'b000;

OUTABCn $<=3$ 'b000 ;

end

else

begin

pwm_a $<=0 ;$

pwm_b $<=0$;

pwm_c $<=0$;

pwm_an $<=0$;

pwm_bn $<=0$;

pwm_cn $<=0$;

end 


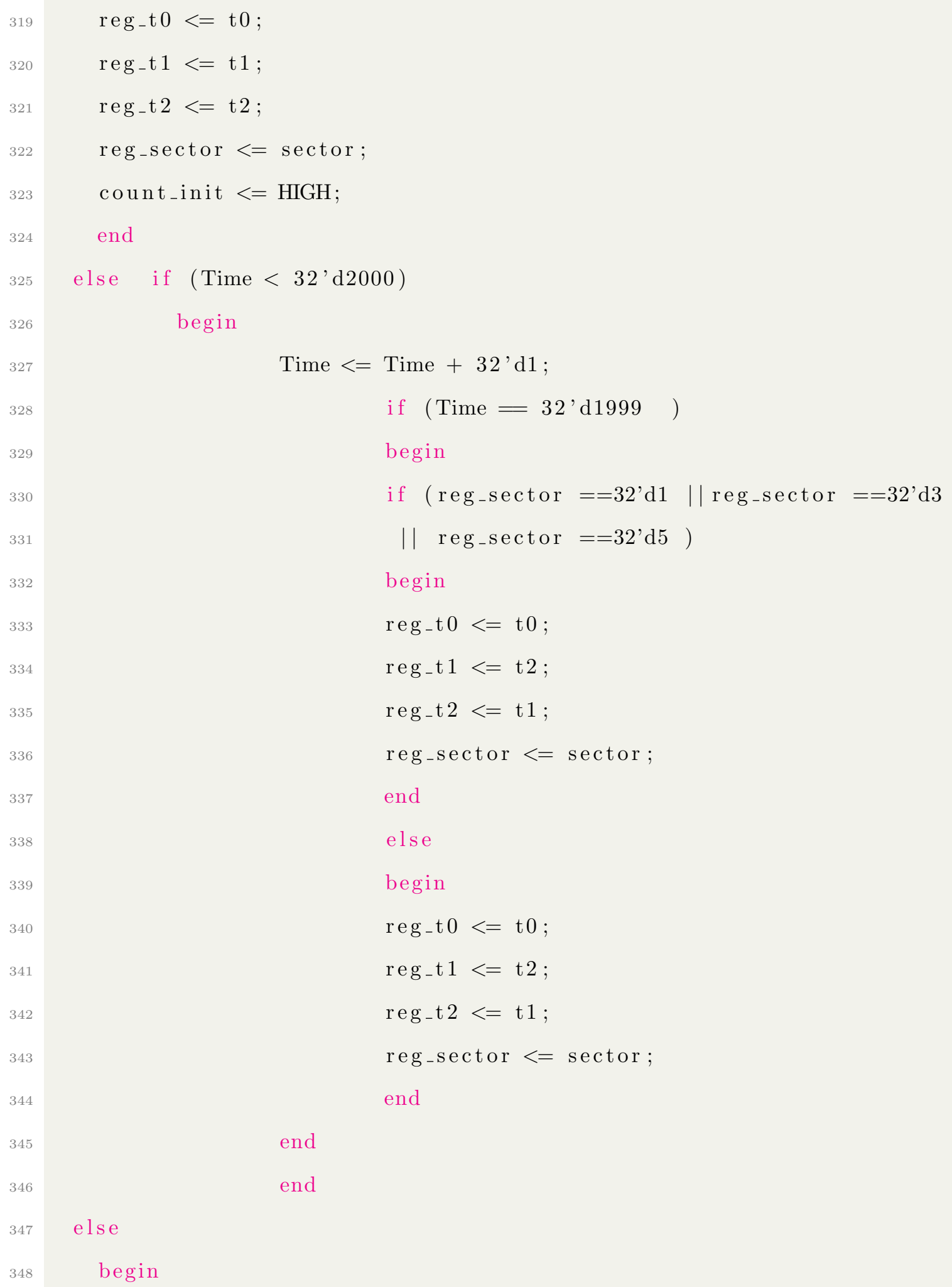

reg_t $0<=$ t 0

reg_t $1<=\mathrm{t} 1$

reg_t $2<=$ t $2 ;$

reg_sector $<=$ sector ;

count_init $<=$ HIGH;

end

else if (Time $<32^{\prime}$ d2000)

begin

Time $<=$ Time $+32^{\prime} \mathrm{d} 1$;

if (Time $=32$ 'd1999 )

begin

if $\left(\mathrm{reg}_{-}\right.$sector $^{\prime}==32^{\prime} \mathrm{d} 1 \quad||$ reg_sector $_{\text {- }}==32^{\prime} \mathrm{d} 3$

|| reg_sector $==32^{\prime} \mathrm{d} 5$ )

begin

reg_t $0<=$ t 0

reg_t $1<=\mathrm{t} 2$

reg t $_{2}<=\mathrm{t} 1$

reg $_{-}$sector $<=$sector

end

else

begin

reg_t $0<=$ t 0

reg t $1<=\mathrm{t} 2$

reg_t $2<=$ t 1

reg_sector $<=$ sector

end

end

end

else

begin 


$349 \quad$ Time $<=32$ 'd 1 ;
$350 \quad$ end
351 endmodule

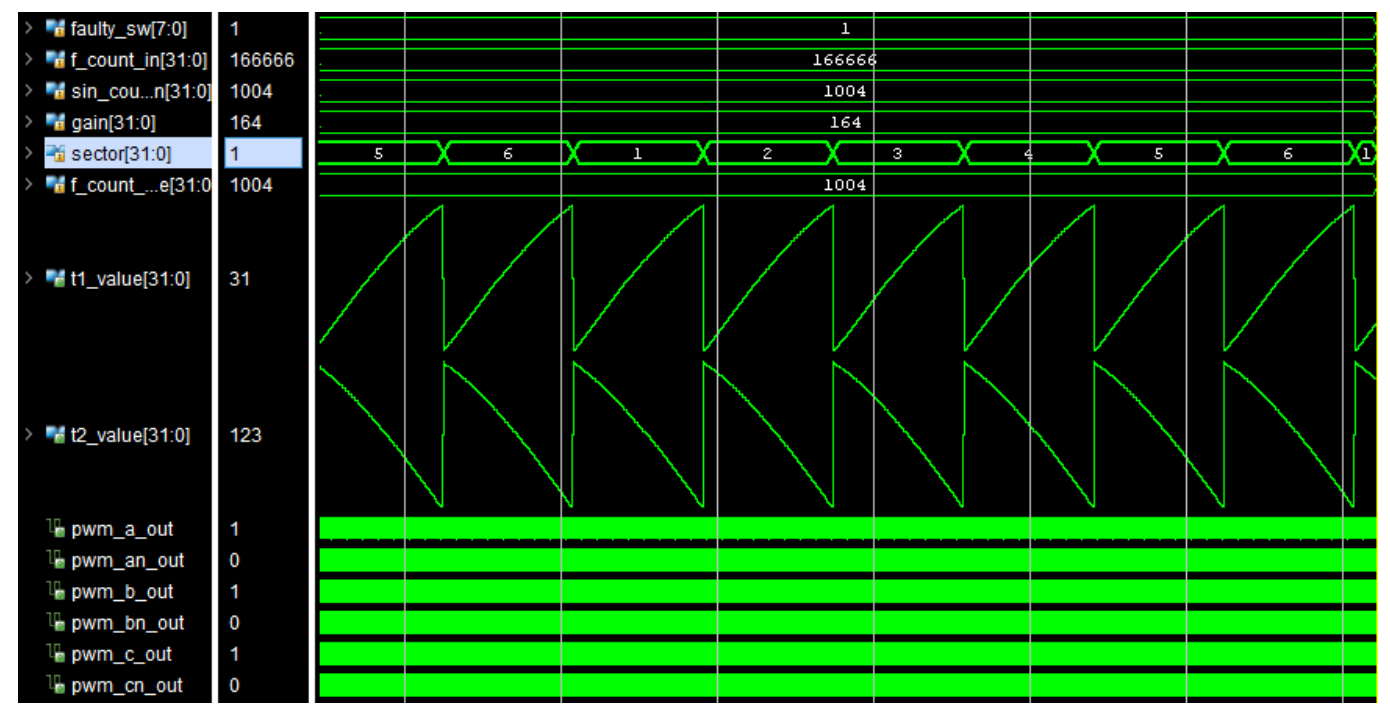

Figure B.1: The screenshot of the simulation of the module 


\section{APPENDIX C}




\section{Reliability Tests for five-phase}
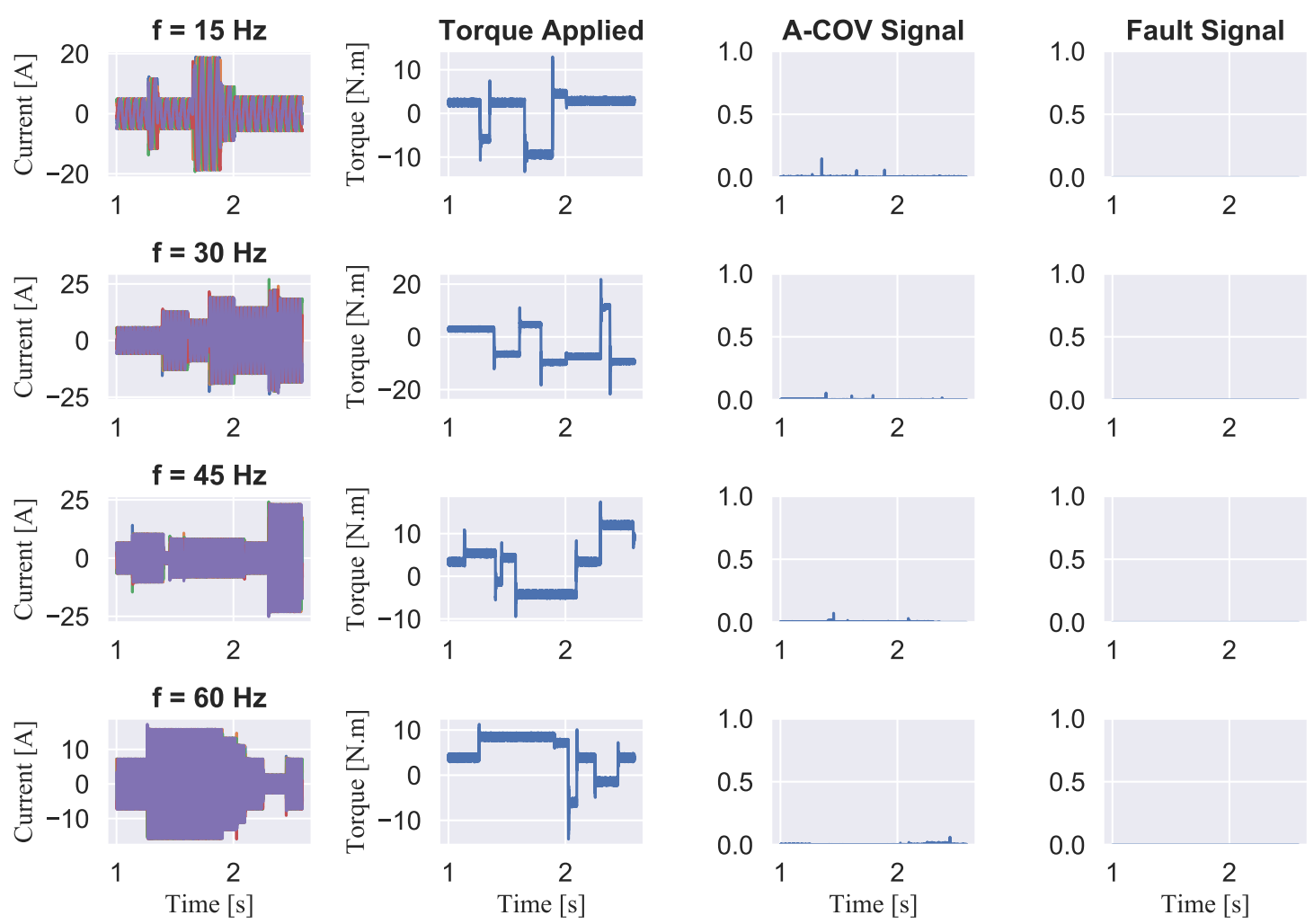

Figure C.1: Reliability no. 2: simulations of random torque commands at random times 

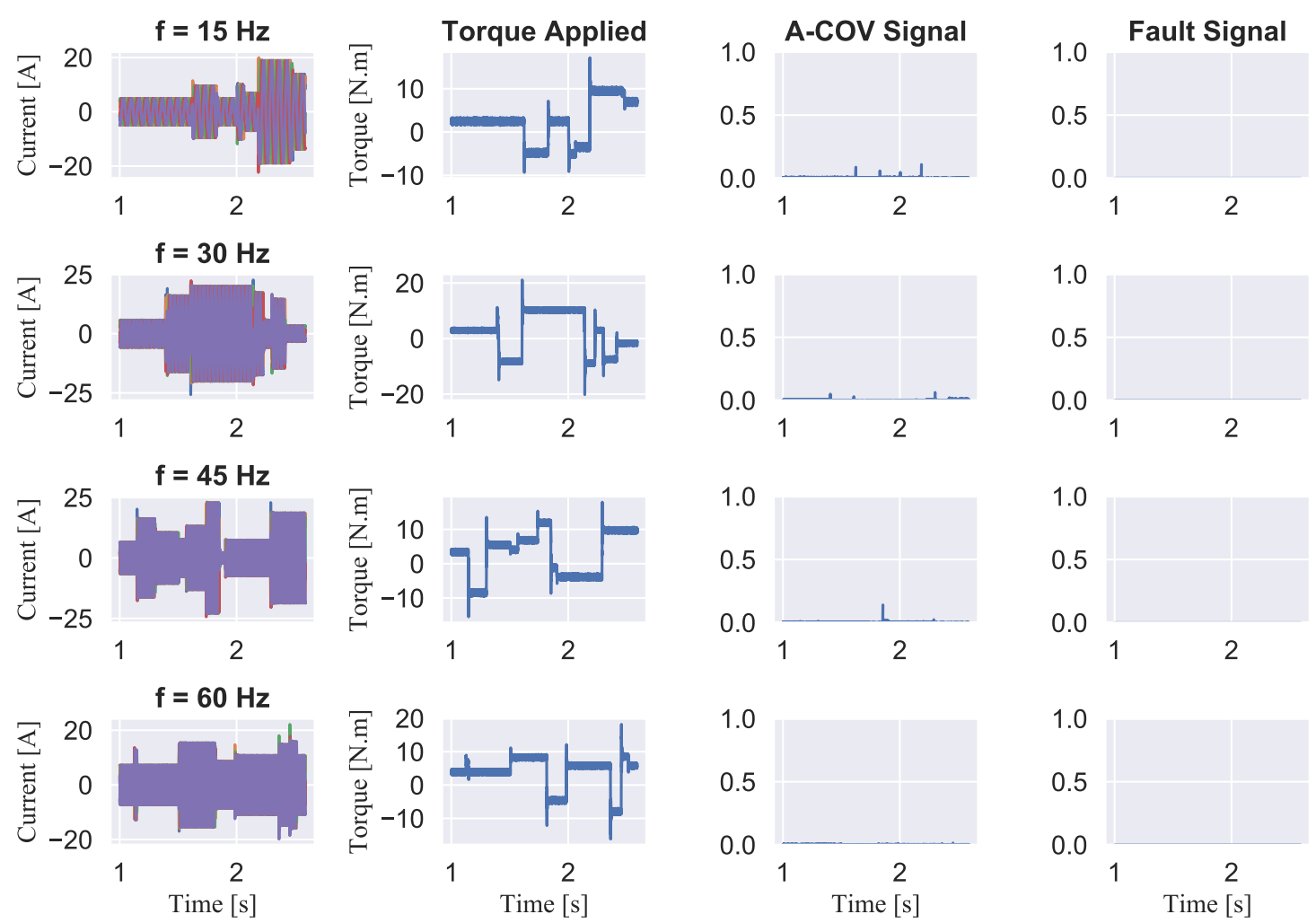

Figure C.2: Reliability no. 3: simulations of random torque commands at random times 

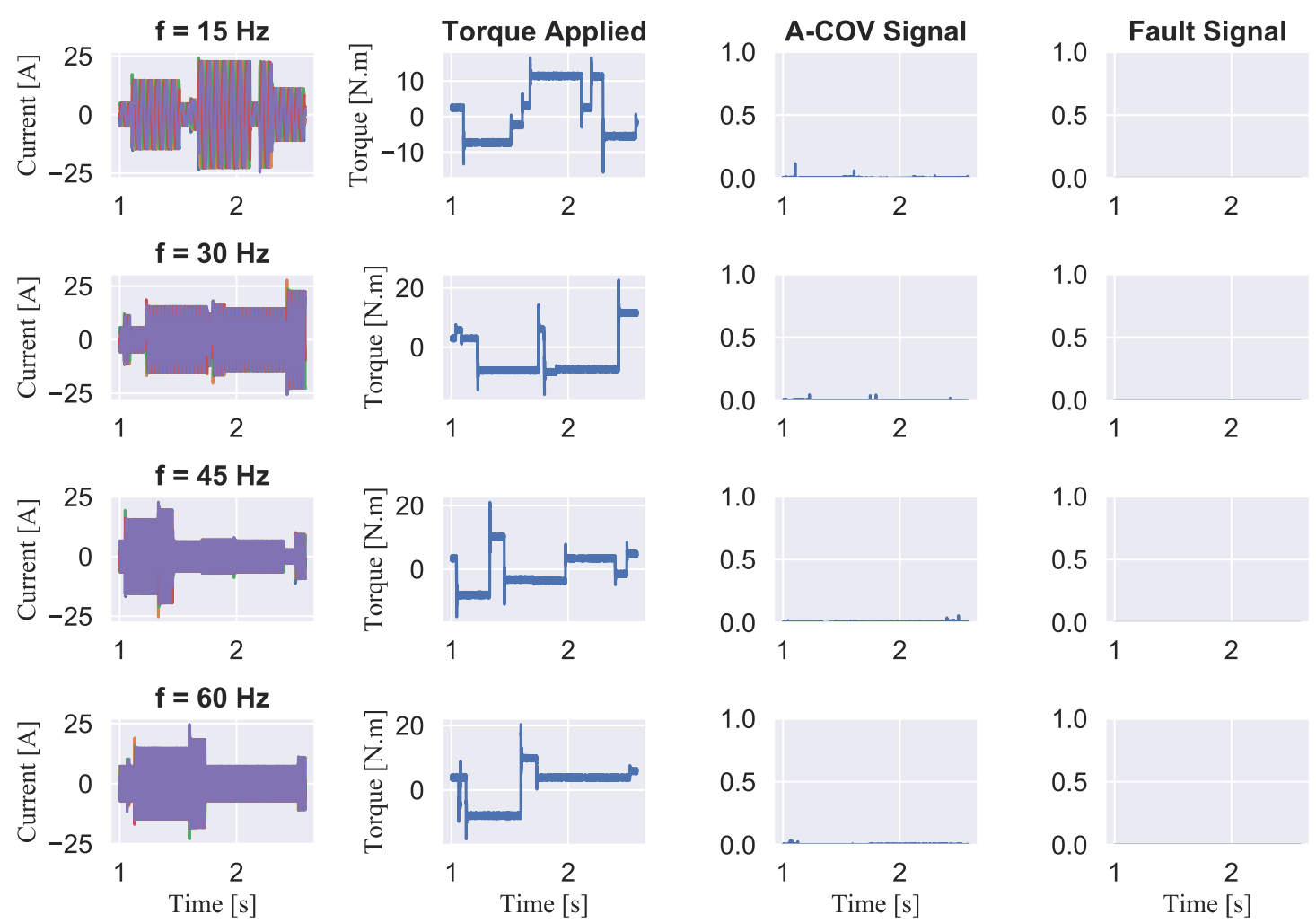

Figure C.3: Reliability no. 4: simulations of random torque commands at random times 

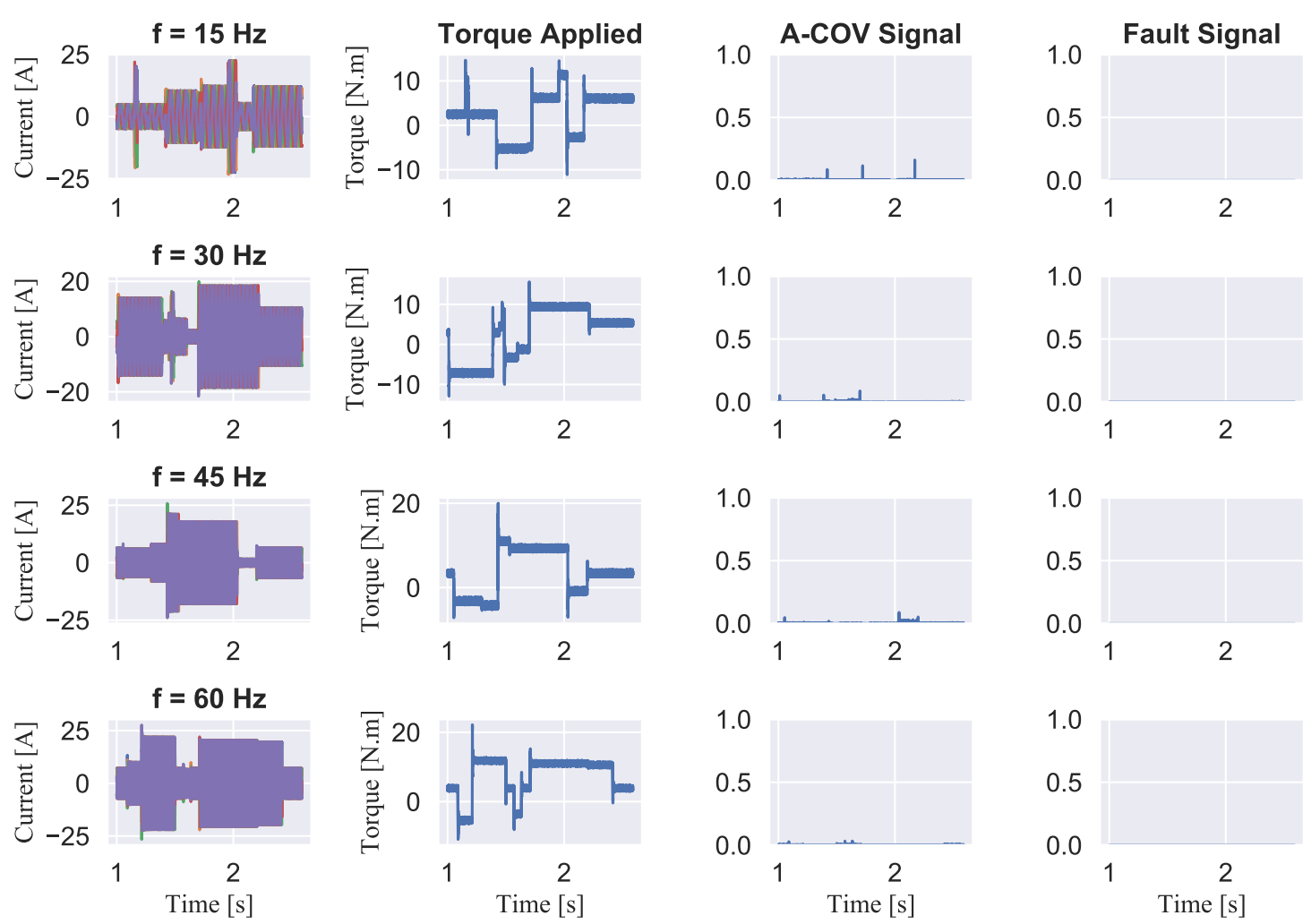

Figure C.4: Reliability no. 5: simulations of random torque commands at random times 


\section{REFERENCES}

[1] A. Julian, G. Oriti, and T. Lipo, "Elimination of common-mode voltage in threephase sinusoidal power converters," Power Electronics, IEEE Transactions on, vol. 14, no. 5, pp. 982-989, Sep 1999.

[2] B. MIRAFZAL, "Survey of fault-tolerance techniques for three-phase voltage source inverters," Industrial Electronics, IEEE Transactions on, vol. 61, no. 10, pp. 5192-5202, Oct 2014.

[3] F. Fuchs, "Some diagnosis methods for voltage source inverters in variable speed drives with induction machines - a survey," in Industrial Electronics Society, 2003. IECON '03. The 29th Annual Conference of the IEEE, vol. 2, Nov 2003, pp. 1378-1385 Vol.2.

[4] T. Elch-Heb and J. Hautier, "Remedial strategy for inverter-induction machine system faults using two-phase operation," in Power Electronics and Applications, 1993., Fifth European Conference on, Sep 1993.

[5] B. Welchko, T. Lipo, T. Jahns, and S. Schulz, "Fault tolerant three-phase AC motor drive topologies: a comparison of features, cost, and limitations," Power Electronics, IEEE Transactions on, vol. 19, no. 4, pp. 1108-1116, July 2004.

[6] S. Bolognani, M. Zordan, and M. Zigliotto, "Experimental fault-tolerant control of a PMSM drive," Industrial Electronics, IEEE Transactions on, vol. 47, no. 5, pp. 1134-1141, Oct 2000.

[7] D. Mohammadi and S. Ahmed-Zaid, "Active common-mode voltage reduction in a fault-tolerant three-phase inverter," in 2016 IEEE Applied Power Electronics Conference and Exposition (APEC), March 2016, pp. 2821-2825.

[8] P. C. Krause, O. Wasynczuk, S. D. Sudhoff, and S. Pekarek, Front Matter. Wiley-IEEE Press, 2013, pp. 608-. [Online]. Available: http: //ieeexplore.ieee.org/xpl/articleDetails.jsp?arnumber $=6739385$

[9] J. Holtz, "Pulsewidth modulation for electronic power conversion," Proceedings of the IEEE, vol. 82, no. 8, pp. 1194-1214, Aug 1994. 
[10] K. Zhou and D. Wang, "Relationship between space-vector modulation and three-phase carrier-based PWM: a comprehensive analysis [three-phase inverters]," IEEE Trans. Ind. Electron., vol. 49, no. 1, pp. 186-196, Feb 2002.

[11] H. Van der Broeck, H.-C. Skudelny, and G. Stanke, "Analysis and realization of a pulsewidth modulator based on voltage space vectors," IEEE Trans. Ind. Appl., vol. 24, no. 1, pp. 142-150, Jan 1988.

[12] D.-W. Chung, J.-S. Kim, and S.-K. Sul, "Unified voltage modulation technique for real-time three-phase power conversion," IEEE Transactions on Industry Applications, vol. 34, no. 2, pp. 374-380, March 1998.

[13] A. M. Hava, R. J. Kerkman, and T. A. Lipo, "Simple analytical and graphical methods for carrier-based pwm-vsi drives," IEEE Transactions on Power Electronics, vol. 14, no. 1, pp. 49-61, Jan 1999.

[14] H. Akagi and T. Shimizu, "Attenuation of conducted EMI emissions from an inverter-driven motor," Power Electronics, IEEE Transactions on, vol. 23, no. 1, pp. 282-290, Jan 2008.

[15] D. Busse, J. Erdman, R. Kerkman, D. Schlegel, and G. Skibinski, "Bearing currents and their relationship to PWM drives," Power Electronics, IEEE Transactions on, vol. 12, no. 2, pp. 243-252, Mar 1997.

[16] Y. Song and B. Wang, "Survey on reliability of power electronic systems," Power Electronics, IEEE Transactions on, vol. 28, no. 1, pp. 591-604, Jan 2013.

[17] Z. Yang, X. Xu, S. Xu, and J. Zhao, "Research on eliminating common-mode voltage of cascaded high-voltage inverter based on svm," in Power and Energy Engineering Conference (APPEEC), 2010 Asia-Pacific, March 2010, pp. 1-4.

[18] M. Swamy, K. Yamada, and T. Kume, "Common mode current attenuation techniques for use with PWM drives," Power Electronics, IEEE Transactions on, vol. 16, no. 2, pp. 248-255, Mar 2001.

[19] X. Dianguo, G. Qiang, and W. Wei, "Design of a passive filter to reduce commonmode and differential-mode voltage generated by voltage-source PWM inverter," in IEEE Industrial Electronics, IECON 2006 - 32nd Annual Conference on, Nov 2006, pp. 2483-2487.

[20] Z. Liu, J. Liu, and J. Li, "Modeling, analysis, and mitigation of load neutral point voltage for three-phase four-leg inverter," Industrial Electronics, IEEE Transactions on, vol. 60, no. 5, pp. 2010-2021, May 2013. 
[21] Y.-S. Lai, "Investigations into the effects of pwm techniques on common mode voltage for inverter-controlled induction motor drives," in Power Engineering Society 1999 Winter Meeting, IEEE, vol. 1, Jan 1999, pp. 35-40 vol.1.

[22] R. Tallam, R. Kerkman, D. Leggate, and R. Lukaszewski, "Common-mode voltage reduction PWM algorithm for AC drives," Industry Applications, IEEE Transactions on, vol. 46, no. 5, pp. 1959-1969, Sept 2010.

[23] Y.-S. Lai and F.-S. Shyu, "Optimal common-mode voltage reduction PWM technique for inverter control with consideration of the dead-time effects-part I: basic development," Industry Applications, IEEE Transactions on, vol. 40, no. 6, pp. 1605-1612, Nov 2004.

[24] A. Hava and E. Un, "A high-performance PWM algorithm for common-mode voltage reduction in three-phase voltage source inverters," Power Electronics, IEEE Transactions on, vol. 26, no. 7, pp. 1998-2008, July 2011.

[25] K. Li, T. Lu, Z. Zhao, L. Yin, F. Liu, and L. Yuan, "Carrier based implementation of reduced common mode voltage PWM strategies," in ECCE Asia Downunder (ECCE Asia), 2013 IEEE, June 2013, pp. 578-584.

[26] Y. Zhang, G. Kuang, and L. Long, "Research on reduced common-mode voltage nonzero vector pulse width modulation technique for three-phase inverters," in Power Elect. and Motion Control Conference (IPEMC), 2012 7th Int., vol. 4, June 2012.

[27] W. Cao, K. Liu, Y. Ji, Y. Wang, and J. Zhao, "Design of a four-branch lcl-type grid-connecting interface for a three-phase, four-leg active power filter," Energies, vol. 8, no. 3, pp. 1606-1627, 2015. [Online]. Available: http://www.mdpi.com/1996-1073/8/3/1606

[28] A. Mendes and A. Cardoso, "Voltage source inverter fault diagnosis in variable speed AC drives, by the average current park's vector approach," in Electric Machines and Drives, 1999. International Conference IEMD '99, May 1999, pp. 704-706.

[29] K. Rothenhagen and F. Fuchs, "Performance of diagnosis methods for IGBT open circuit faults in three phase voltage source inverters for AC variable speed drives," in Power Electronics and Applications, 2005 European Conference on, Sept 2005, pp. 10 pp.-P.7.

[30] R. Peuget, S. Courtine, and J. P. Rognon, "Fault detection and isolation on a PWM inverter by knowledge-based model," IEEE Transactions on Industry Applications, vol. 34, no. 6, pp. 1318-1326, Nov 1998. 
[31] M. Trabelsi, M. Boussak, and M. Gossa, "Multiple IGBTs open circuit faults diagnosis in voltage source inverter fed induction motor using modified slope method," in Electrical Machines (ICEM), 2010 XIX International Conference on, Sept 2010, pp. 1-6.

[32] O.-S. Yu, N. ju Park, and D. seok Hyun, "A novel fault detection scheme for voltage fed PWM inverter," in IEEE Industrial Electronics, IECON 2006 - 32nd Annual Conference on, Nov 2006, pp. 2654-2659.

[33] T. Orlowska-Kowalska and P. Sobanski, "Simple sensorless diagnosis method for open-switch faults in SVM-VSI-fed induction motor drive," in Industrial Electronics Society, IECON 2013 - 39th Annual Conference of the IEEE, Nov 2013, pp. 8210-8215.

[34] P. Sobanski and T. Orlowska-Kowalska, "Experimental validation of the simple voltage-vector-location-based method of open-circuit igbts faults in dtc-svm induction motor drive," in Electrical Drives and Power Electronics (EDPE), 2015 International Conference on, Sept 2015, pp. 19-24.

[35] M. Masrur, Z. Chen, B. Zhang, and Y. Murphey, "Model-based fault diagnosis in electric drive inverters using artificial neural network," in Power Engineering Society General Meeting, 200\%. IEEE, June 2007, pp. 1-7.

[36] M. Alavi, M. Luo, D. Wang, and H. Bai, "IGBT fault detection for three phase motor drives using neural networks," in Emerging Technologies Factory Automation (ETFA), 2012 IEEE 17th Conference on, Sept 2012, pp. 1-8.

[37] B. Lu and S. Sharma, "A literature review of IGBT fault diagnostic and protection methods for power inverters," Industry Applications, IEEE Transactions on, vol. 45, no. 5, pp. 1770-1777, Sept 2009.

[38] D. Mohammadi, L. Daoud, N. Rafla, and S. Ahmed-Zaid, "Zynq-based soc implementation of an induction machine control algorithm," in 2016 IEEE 59th International Midwest Symposium on Circuits and Systems (MWSCAS), Oct 2016, pp. 1-4.

[39] O. Buchholz, and J. Boecker, "FPGA-based Dynamically Reconfigurable Control of Induction Motor Drives," in Industrial Electronics Society, IECON 2013-39th Annual Conference of the IEEE. IEEE, 2013, pp. 3543-3548.

[40] E. Monmasson, and M. N. Cirstea, "FPGA Design Methodology for Industrial Control Systems - A Review," IEEE Transactions on Industrial Electronics, vol. 54, no. 4, pp. 1824-1842, 2007. 
[41] M. Curkovič, K. Jezernik, and R. Horvat, "FPGA-based predictive sliding mode controller of a three-phase inverter," IEEE Transactions on Industrial Electronics, vol. 60, no. 2, pp. 637-644, 2013.

[42] D. H. Lee and J. W. Ahn, "A simple and direct dead-time effect compensation scheme in PWM-VSI," IEEE Transactions on Industry Applications, vol. 50, no. 5, pp. 3017-3025, Sept 2014.

[43] J. Haruna, S. Ikegami, and N. Hoshi, "A dead-time compensation method on a PWM control scheme for a 6 switches two PMSMs drive inverter," in Future Energy Electronics Conference (IFEEC), 2013 1st International, Nov 2013, pp. 143-148.

[44] S. Bolognani, M. Ceschia, P. Mattavelli, A. Paccagnella, and M. Zigliotto, "Improved FPGA-based dead time compensation for SVM inverters," in Power Electronics, Machines and Drives, 2004. (PEMD 2004). Second International Conference on (Conf. Publ. No. 498), vol. 2, March 2004, pp. 662-667 Vol.2.

[45] L. Daoud, D. Zydek, and H. Selvaraj, "A Survey on Design and Implementation of Floating Point Adder in FPGA," in Progress in Systems Engineering. Springer, 2015, pp. 885-892.

[46] I. Bahri, L. Idkhajine, E. Monmasson, and M. E. A. Benkhelifa, "Hardware/software codesign guidelines for system on chip FPGA-based sensorless AC drive applications," IEEE Transactions on Industrial Informatics, vol. 9, no. 4, pp. 2165-2176, Nov 2013.

[47] R. Velzquez, O. Luca, D. Navarro, L. A. Barragn, J. I. Artigas, and C. Sagus, "Design of an FPGA-based full-state feedback controller using high level synthesis tools," in 2014 IEEE 15th Workshop on Control and Modeling for Power Electronics (COMPEL), June 2014, pp. 1-6.

[48] Y. L. Aung, S. K. Lam, and T. Srikanthan, "Hardware-software codesign of EKFbased motor control for domain-specific reconfigurable platform," in Electronic System Design (ISED), 2013 International Symposium on, Dec 2013, pp. 93-97.

[49] L. Daoud, D. Zydek, and H. Selvaraj, "A Survey of High Level Synthesis Languages, Tools, and Compilers for Reconfigurable High Performance Computing," in Advances in Systems Science. Springer, 2014, pp. 483-492, , DOI: 10.1007/978-3-319-01857-7_47.

[50] L. H. Crockett,R. A. Elliot, M. A. Enderwitz, and R. W. , The Zynq Book: Embedded Processing with the Arm Cortex-A9 on the Xilinx Zynq-7000 All Programmable SoC. Strathclyde Academic Media, 2014. 
[51] Xilinx Inc., Vivado Design Suite: AXI Reference Guide, June, 2015.

[52] D. Mohammadi, N. Rafla, and S. Ahmed-Zaid, "Design considerations for an optimized FPGA implementation of space-vector PWM for a two-level inverter," in 2016 IEEE Transportation Electrification Conference and Expo (ITEC), June 2016, pp. 1-5.

[53] Xilinx Inc., ZC702 Evaluation Board for the Zynq-7000 XC7Z020 User Guide, September, 2015. 